\title{
Advances in Optics and Photonics
}

\section{Concepts in quantum state tomography and classical implementation with intense light: a tutorial}

\author{
Ermes Toninelli, ${ }^{1, \dagger}$ Bienvenu Ndagano, ${ }^{2, \dagger} \oplus$ Adam Vallés, ${ }^{2} @$ \\ Bereneice Sephton, ${ }^{2}$ IsaAc Nape, ${ }^{2}$ Antonio Ambrosio, ${ }^{3}{ }^{\circ}$

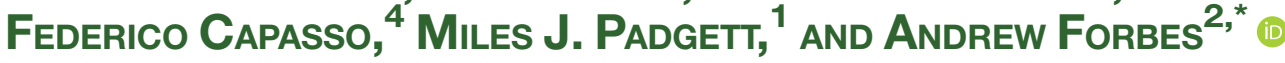

${ }^{1}$ SUPA, School of Physics and Astronomy, University of Glasgow, Glasgow G12 8QQ, UK

${ }^{2}$ School of Physics, University of the Witwatersrand, Private Bag 3, Johannesburg 2050, South Africa

${ }^{3}$ Center for Nanoscale Systems, Harvard University, Cambridge, Massachusetts 02138, USA

${ }^{4}$ Harvard John A. Paulson School of Engineering and Applied Sciences, Harvard University, Cambridge, Massachusetts 02138, USA

*Corresponding author: andrew.forbes@wits.ac.za

Received August 31, 2018; revised November 30, 2018; accepted December 3, 2018; published March 7, 2019 (Doc. ID 344254)

A tomographic measurement is a ubiquitous tool for estimating the properties of quantum states, and its application is known as quantum state tomography (QST). The process involves manipulating single photons in a sequence of projective measurements, often to construct a density matrix from which other information can be inferred, and is as laborious as it is complex. Here we unravel the steps of a QST and outline how it may be demonstrated in a fast and simple manner with intense (classical) light. We use scalar beams in a time reversal approach to simulate the outcome of a QST and exploit non-separability in classical vector beams as a means to treat the latter as a "classically entangled" state for illustrating QSTs directly. We provide a complete do-it-yourself resource for the practical implementation of this approach, complete with tutorial video, which we hope will facilitate the introduction of this core quantum tool into teaching and research laboratories alike. Our work highlights the value of using intense classical light as a means to study quantum systems and in the process provides a tutorial on the fundamentals of QSTs. (C) 2019 Optical Society of America

https://doi.org/10.1364/AOP.11.000067

1. Introduction. . . . . . . . . . . . . . . . . . . . . . . . . 69

1.1. Basic Concept. . . . . . . . . . . . . . . . . . . . . . . . . . 69

1.2. Brief Historical Review . . . . . . . . . . . . . . . . . . 70

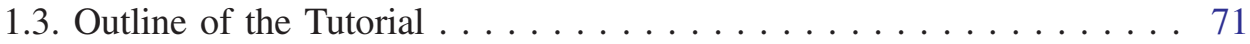

2. QST of Two-Level States . . . . . . . . . . . . . . . . . . 72

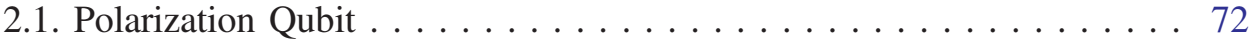


2.2. Spatial Mode Qubit . . . . . . . . . . . . . . . . . . . . . . 78

2.3. Biphoton Qubits . . . . . . . . . . . . . . . . . 83

2.4. Extracting Information from QST Measurements . . . . . . . . . . 91

3. Simulating QST Measurements with Scalar Light . . . . . . . . . . . . 93

4. QSTS with Classically Entangled Light . . . . . . . . . . . . . . 96

4.1. What is Entanglement? . . . . . . . . . . . . . . . . . . . 98

4.2. Non-Separability, Vector Beams, and Classical Entanglement . . . . . . 98

4.3. Controlled Classical Entanglement by Spin-Orbit Coupling . . . . . . 100

4.4. Exploiting the Mathematical Similarity . . . . . . . . . . . . . . . . . . 104

4.5. Measurement in a Classical QST . . . . . . . . . . . . . . . . 106

4.6. Bell Measurement with Classical Light . . . . . . . . . . . . . . . . . 109

4.7. Experimental Demonstration . . . . . . . . . . . . . . . . 110

5. DIY Laboratory Implementation . . . . . . . . . . . . . . . . 118

5.1. 3D-Printed Roto-Flip Stages . . . . . . . . . . . . . . . . . . 118

5.2. Video Demonstration of the Automated State-Tomography System in

Action . . . . . . . . . . . . . . . . . . . . . . . 120

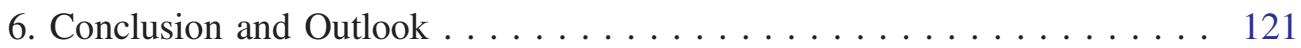

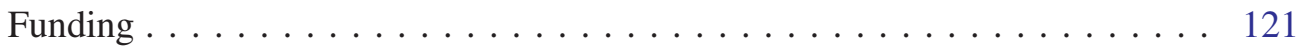

Acknowledgment. . . . . . . . . . . . . . . . . . . 121

References . . . . . . . . . . . . . . . . . . . . . . . 121 


\title{
Concepts in quantum state tomography and classical implementation with intense light: a tutorial
}

\author{
Ermes Toninelli, Bienvenu Ndagano, Adam Vallés, Bereneice \\ Sephton, Isaac Nape, Antonio Ambrosio, Federico Capasso, \\ Miles J. Padgett, and Andrew Forbes
}

\section{INTRODUCTION}

\subsection{Basic Concept}

One of the challenges in quantum optics is to unravel an unknown state [1-3], with the difficulty arising primarily from the measurement problem in quantum mechanics $[4,5]$. First, a measurement destroys the information of the state, or at the very least perturbs it [6], negating the possibility of performing multiple measurements on the same state [7]. Neither is it possible to clone the state one wishes to study: this implies that measurements cannot be performed on exact copies of a state [8]. Moreover, a state is generally unknown-it could be pure or mixed, high dimensional or low dimensional - and so the choice of basis and measurement sequence is not trivial [9-12]. Consequently, we can infer only a little information at a time by probing a particular aspect of a quantum state [13]. In other words, only one question can be asked (by performing a measurement) from which we get one piece of information (the measurement's outcome). The standard approach for collecting information about a quantum state is to perform multiple tomographic measurements, so-called projective measurements, in what is known as a quantum state tomography (QST) [see Refs. [1,14] for good reviews]. A QST is very similar to the well-known computed tomography or "CT" scans in medicine: once many projective measurements are made, each probing a particular aspect of the possible state, the complete quantum state is built up through a tomographic process. In essence, each unknown quantum state is "sliced" and completely characterized through a series of projective measurements in different bases, retrieving the information of a new dimension for each measurement [15]. It is akin to building up an image of a complex object by making only simple projections of its shadow, as illustrated in Fig. 1. In this picture, the unknown shape of an object (i.e., the unknown state) can be worked out by the information contained in the measured shadows (i.e., the results of projection measurements). In the quantum case, the outcome is the complete set of observables whose probabilistically weighted outcome fully describes the quantum state [16]. This then becomes an inverse problem: knowing the outcome of every question (the outcomes of our measurements), can we work out what the "object" is? In the quantum world this often translates into determining a density matrix for the quantum state from which all other required information can be inferred [15,17,18].

QSTs (plural because they come in various guises) are time consuming and complex: the number of measurements does not scale well with the dimension of the quantum state [19,20], noise affects the outcome [21-24], and one must assume that identical states are produced at the source, e.g., identical copies of photons from a spontaneous parametric downconversion (SPDC) process [25]. Consider systems with $N$ photons, each in $d$ dimensional states. The total dimension of the system is then $D=d^{N}$ and 
can be described by a density matrix of $d^{2 N}-1$ independent real parameters. For example, to characterize a $N$-qubit $(d=2)$ state one requires $2^{2 N}$ measurements of different observables [26,27], with each one performed more than once to accumulate reasonable statistics, thus scaling unfavorably (exponentially) with the system dimension. Often an over-complete tomographic measurement is performed [28,29], i.e., more measurements than just the minimum required. For example, an overcomplete tomographic measurement of a two-photon $(N=2)$ high-dimensional qudit state of dimension $d$ would require $[d(d+1)]^{2}$ measurements [30]. This is usually done for accuracy, and in the rest of this tutorial we too will use over-complete sets of measurements even though less would often suffice. Once the measurements are complete, the reconstruction itself can be computationally intensive, as solving inverse problems is not easy. For this reason there are many ingenious approaches to reduce the number of measurements needed, or to extract as much information as possible by a judicious choice of measurement [31-34]. To return to our shadow analogy: How many projections do we need and what should they be to quickly find the object? Addressing these issues remains a topic of active research: making a QST state independent, fast, robust, and compact for on-chip deployment [35-37].

\subsection{Brief Historical Review}

A QST as we know it today was introduced in the late 1980s to obtain the Wigner distribution by tomographic measurements of quadrature amplitudes [38], taking into account the direct correspondence between the Wigner function and the density matrix of any desired quantum state $[39,40]$. Other methods to determine a quantum state were proposed $[41,42]$, studying also the open question of the impossibility to determine the probability distributions of a superposition quantum state with a direct measurement [43].

Tomographic measurements have been used to characterize a myriad of quantum states [44-50]. Importantly, QST has become essential in the characterization of

\section{Figure 1}

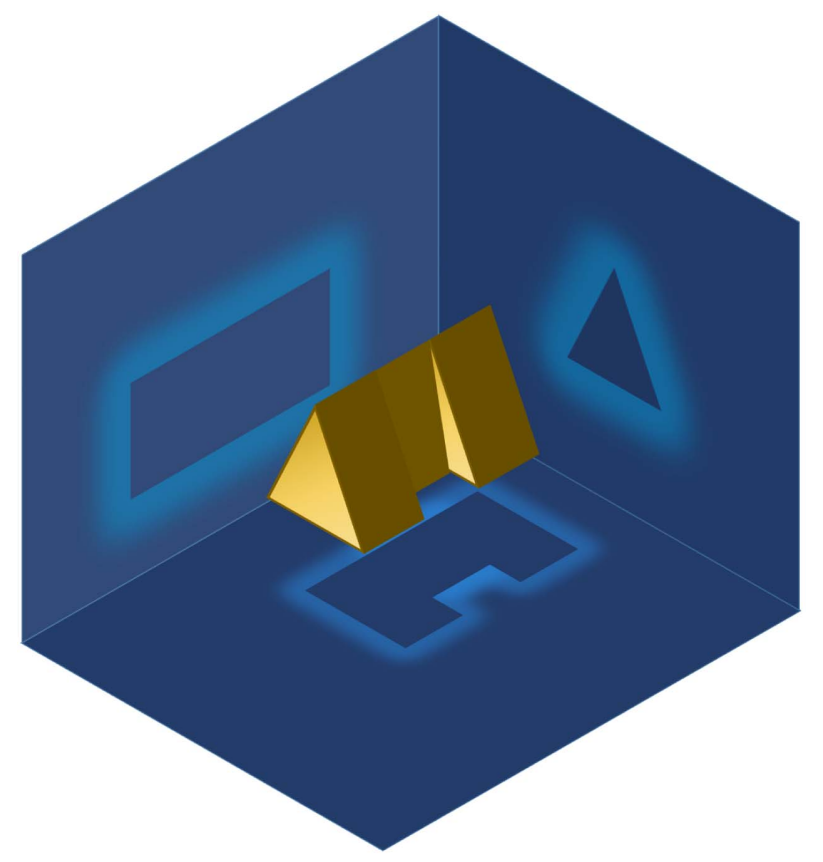

QST attempts to reconstruct a potentially complex quantum state by a series of simple projective measurements. This is analogous to trying to reconstruct a complicated object by considering only its shadow from various angles. 
entanglement sources, e.g., entanglement sources using photons [51-57], atoms [58,59], and even with molecular vibrational modes [60]. However, the polarization of a photon is the most common known degree of freedom used to encode a quantum state. This is due to the great variety and availability of high-efficiency polarization control elements. Consequently, many experimental milestones in quantum optics have been accomplished by using the polarization degree of freedom [61-64].

Although QSTs have been performed on many quantum systems, in this tutorial we will consider photonic quantum states, exploiting the spatial mode and polarization degrees of freedom in light. QSTs have been performed extensively on the latter (see, for example, Refs. $[53,56,65,66])$, while other degrees of freedom are becoming more prevalent these days, e.g., entangled spatial modes [67-72] for improved communication security [73-76] and high-dimensional entanglement [77-80]. Although highdimensional quantum state generation is increasingly relevant, the characterization of such systems is extremely complex due to the exponential increase of projections with dimension. Nevertheless, QSTs have been successfully demonstrated on high-dimensional spatial mode entanglement by using various projection approaches [81-83].

There are also advantages when mixing two degrees of freedom in the same photon, also known as hybrid states [84-86]. One pertinent example that we will consider in more detail in this tutorial is that of hybrid polarization and orbital angular momentum (OAM) degrees of freedom [87]; they are relevant as natural modes of optical fibers [88] and free space [89]. The generation of such states can be achieved by using holograms [90], but also in a more straightforward way by using spin-orbit conversion devices based on liquid crystals [91], or even based on metamaterial technology $[92,93]$. On the other hand, instead of the most general case of generating entanglement between two photons, we can study entanglement between two degrees of freedom in a single photon [94,95], paving the way for the use of quantum measurement tools to characterize states generated with intense laser beams [96-98].

\subsection{Outline of the Tutorial}

In this tutorial, we will not only outline the core ideas of a QST, but will also show how they may be performed in a fast and easy manner with intense classical light. Classical laser light does not suffer from the aforementioned quantum measurement woes: one can make as many measurements as one likes simultaneously on the same intense light field. The use of classical light in quantum studies is not new [99-101], while today there is a growing realization that non-separability, the quintessential property of quantum entanglement, is not unique to quantum mechanics [102-106]. As such, many classical systems exhibit properties usually associated with quantum entangled states [107-109], also referred as nonquantum entanglement [110,111], or non-separable states [112,113]. However, even though a particular classical system can simulate most of the features of entanglement, it fails to simulate quantum nonlocality [114]. In relation to QSTs, classical light has been exploited as an alignment and predictive tool using the so-called backprojection with single photons [115-121], following the time reversal analogy given by Klyshko [122].

We exploit these similarities between some classical and quantum states to develop a useful laboratory tool for teaching and demonstrating QSTs. First we outline the general principles of quantum tomographic measurements, explaining in tutorial fashion how to translate theory into experiment, how to perform the measurements, and how to extract the required information. Next, we explain how to use the time reversal concept to mimic the quantum system using backprojected scalar light. This allows one to perform the measurement as if there were entangled photons. To actually use the quantum toolbox directly, we make use of non-separable classical light, exploiting 
the fact that non-separability is the hallmark of quantum entangled systems. We use vector vortex beams as our non-separable states of light, depicted on a high-order Poincaré sphere [123-125], and perform a QST with standard optical components. This allows us to create any state from fully separable to fully non-separable, and treat it as a controlled source of entanglement, albeit entirely classical and with intense laser light. As a consequence, however, the possibility of studying the quantum nonlocality is discarded. We show that we can easily reconstruct any quantum state by automating the optical elements that perform each particular projection. We demonstrate all the measurements typically performed in a quantum laboratory, such as the Bell inequality measurements, but here with intense laser beams. We provide the complete toolkit, from the software to the three-dimensional (3D) designs (see Ref. [126]), for others to duplicate as a versatile teaching tool that can be 3D printed and automated with all the designs and software used to obtain the results shown in this tutorial, as well as a video (see Visualization 1 [126]) demonstrating the process in action. We hope that this tutorial and associated resource will inspire the teaching of quantum mechanics from an experimental perspective, a component sorely lacking in many quantum courses, and be of value in realizing educational and research objectives alike.

\section{QST OF TWO-LEVEL STATES}

\subsection{Polarization Qubit}

The quantum bit (qubit) is the fundamental unit of quantum information. Unlike a classical bit that assumes one of two distinct states, 0 or 1 , the quantum bit is a weighted superposition of two orthogonal states of a given degree of freedom, for example,

$$
|\psi\rangle=\alpha|0\rangle+\beta|1\rangle
$$

with probability amplitudes $\alpha$ and $\beta$ so that $|\alpha|^{2}+|\beta|^{2}=1$. Spin states are the most common example of qubits currently explored to realize quantum computation and communication. For photons, these spin states can correspond to left- and right-circular polarization states [127]. In general, one can express the state of a polarization qubit as

$$
|\psi\rangle=\cos (\theta / 2)|R\rangle+\exp (i \varphi) \sin (\theta / 2)|L\rangle,
$$

where $|R\rangle$ and $|L\rangle$ represent the right- and left-circular polarization states, respectively. The parameter $\varphi \in[0,2 \pi]$ is related to the phase difference between the polarization states, while $\theta$ defines the weighting factor. In the special cases of $\theta=0$ and $\theta=\pi$, the qubit state $|\psi\rangle$ corresponds to the polarization eigenstates $|R\rangle$ and $|L\rangle$, respectively. By manipulating both $\theta$ and $\varphi$, one can produce arbitrary polarization states. For example, for $\theta=\pi / 2$, one can prepare any linear polarization state as depicted graphically in Table 1 .

The definition of the qubit state in Eq. (2) has an intuitive representation as a point on a sphere, commonly referred to as the Poincaré sphere. Historically the Poincaré sphere is the name used when describing classical polarization states, while the Bloch sphere is used when the polarization is regarded as a quantum state. Nevertheless, they describe the same two-dimensional space. First let us look at the effect of tuning the probability amplitudes, by varying $\theta$. Normalization of the quantum state requires

Table 1. Linear Polarization States Produced for $\theta=\pi / 2$

\begin{tabular}{lcccc}
\hline Phase $\varphi$ & 0 & $\pi / 2$ & $\pi$ & $3 \pi / 2$ \\
\hline Qubit state $|\psi\rangle$. & $\leftrightarrow$ & $\searrow$ & $\uparrow$ & $\nearrow$ \\
\hline
\end{tabular}


conservation of probability; that is, the probability of finding the qubit $|\psi\rangle$ in any of its eigenstates must be 1 (the qubit must be in some state after all). Mathematically, this is expressed as

$$
|\langle\psi \mid \psi\rangle|^{2}=\left|\cos ^{2}(\theta / 2)\right|^{2}+\sin ^{2}(\theta / 2)=1 .
$$

By visual examination, one notices that Eq. (3) describes a circle of radius 1, where $\theta$ parameterizes the position on the circle, as shown in Fig. 2(a). Let us refer to this as the amplitude circle.

The phase term $\exp (i \varphi)$ is an oscillatory function, whose variable $\varphi$ can be bounded in the interval $[0,2 \pi]$. By plotting this oscillatory function on the complex plane, one realizes that the angle $\varphi$ maps to an angular position on a unit circle. Varying $\varphi$ changes the relative phase between the basis states, resulting in a rotation of the qubit state on a unit circle, as shown in Fig. 2(b) for linearly polarized states $(\theta=\pi / 2)$. We will refer to this circle as the phase circle.

The Poincaré sphere brings the description of amplitude and phase variation into a single picture. Place a point on the amplitude circle by fixing $\theta$, rotate it around the phase circle by an angle $\varphi$, and one has the recipe for locating a position on the surface of the Poincaré sphere. Thus, an arbitrary qubit state parameterized by a unique set of coordinates $(\theta, \varphi)$ can be mapped to a point on the unit sphere, as shown in Fig. 2(c). In the Poincaré sphere representation, the qubit state lives on the surface of a sphere, and motion on the sphere transforms one qubit state into another. To be useful in a quantum application, it is necessary to be able to manipulate and characterize the qubit state. This requires locating the qubit on the sphere and moving it to a different position. Given that the geometry is spherical, we simply need a reference point on the sphere and a set of rotation transformations about the origin. In three dimensions, one needs a set of rotations about the $x, y$, or $z$ axis, as shown in Fig. 3. Given that the initial state is known, the final state can be uniquely determined by evaluating the influence of the various rotations: this is the objective of a quantum state tomographic measurement (QSTM). But before we delve into the inner workings of a QST, we first need an additional description of qubit states: the density matrix.

\section{Figure 2}

(a)

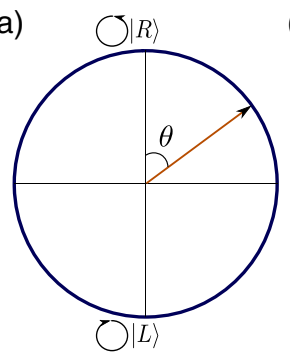

(b)

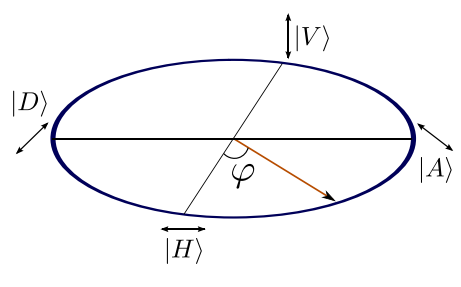

(c)

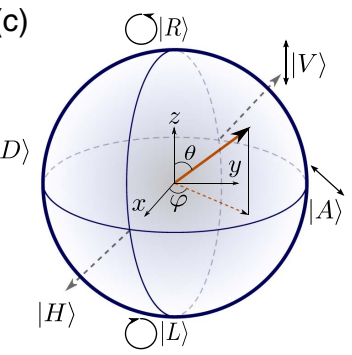

Intuitive description of the Poincaré sphere. (a) Control over the amplitude parameter $\theta$ allows one to continuously change the position of the qubit (indicated by the arrow) on the unit circle, resulting in a change of the relative amplitudes of left- and rightcircular polarizations. (b) Control over the phase parameter $\varphi$ allows one to continuously change the position of the qubit (indicated by the arrow) on a different unit circle, this time resulting in a rotation of the polarization state. This is demonstrated for $\theta=\pi / 2$ in Eq. (2). (c) Simultaneous control of phase and amplitude results in a description of the general qubit in Eq. (2) on a unit sphere where the poles are the polarization states. 
The density matrix description of quantum states is more general than the state vector description of Eq. (2). This is because density matrices allow one to describe the actual outcome of measurements, and pertinently, for both pure and mixed states. Imagine we have an ensemble of $K$ independent single photons, each prepared in the qubit state $\left|\psi_{n}\right\rangle$ with $n=\{1,2, \ldots, K\}$. If all the photons are identical, that is, they are prepared in the same state $\left|\psi_{n}\right\rangle=|\psi\rangle$, then every photon can be described using a single state vector $|\psi\rangle$ as in Eq. (2). The state of every photon is said to be pure. Conversely, if some (or all) of the $K$ photons are prepared in different states, the result is a statistical mixture of pure states. A photon randomly chosen in the mixture can be found to be in a given pure state $\left|\psi_{n}\right\rangle$ with a certain probability. However, because all the photons are independent and uncorrelated, there exists no phase relation between their states. Consequently, the formalism in Eq. (2) cannot appropriately describe such a mixture. A density matrix is a useful tool to overcome this hurdle.

Assume that the state of a single photon in the aforementioned ensemble can be described by a $2 \times 2$ matrix, $\rho$, called the density matrix. In general, $\rho$ can be decomposed in terms of its eigenvectors and eigenvalues:

$$
\rho_{\text {mixed }}=\sum_{m} c_{m}\left|\psi_{m}\right\rangle\left\langle\psi_{m}\right|
$$

In this description each $c_{m}$ is real, positive, and corresponds to the probability of measuring a photon prepared in the eigenstate $\left|\psi_{m}\right\rangle$. Conservation of probabilities requires that $\sum_{m} c_{m}=1$. By construction, density matrices must be Hermitian; that is, $\rho^{\dagger}=\rho$, where superscript $\dagger$, called "dagger," refers to the complex conjugation and transpose operation. This can be easily verified as $(|a\rangle\langle b|)^{\dagger}=|b\rangle\langle a|$.

The density matrix in Eq. (4) describes a statistical mixture of pure qubit states $\left|\psi_{m}\right\rangle\left\langle\psi_{m}\right|$. Hence, we refer to $\rho$ as a mixed state density matrix. In the special case where all photons are identically prepared (pure state), there exists only one eigenstate with unit eigenvalue. The pure state density matrix then reads $\rho_{\text {pure }}=|\psi\rangle\langle\psi|$. For the pure state in Eq. (2), the density matrix is expressed as follows:

$$
\begin{aligned}
\rho= & |\psi\rangle\left\langle\psi\left|=\cos ^{2}(\theta / 2)\right| R\right\rangle\left\langle R\left|+\sin ^{2}(\theta / 2)\right| L\right\rangle\langle L| \\
& +\cos (\theta / 2) \sin (\theta / 2) \exp (-i \varphi)|R\rangle\langle L| \\
& +\cos (\theta / 2) \sin (\theta / 2) \exp (i \varphi)|L\rangle\langle R| .
\end{aligned}
$$

One can now provide a matrix representation of $\rho$ by assigning coordinate vectors to the basis states. For example, assume the following representation of the

\section{Figure 3}
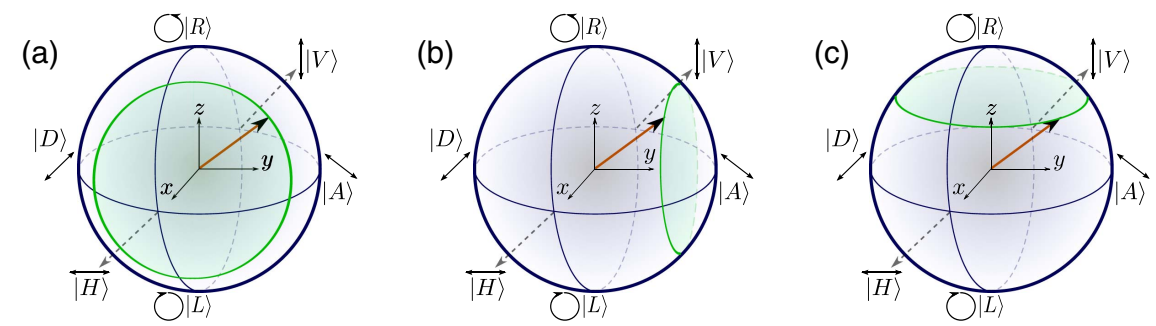

Elementary rotations on the surface of the Poincare sphere. The qubit state, indicated by the position vector colored orange, can be rotated on the surface of the sphere about the (a) $x$ axis, (b) $y$ axis, and (c) $z$ axis. 
polarization states: $|R\rangle \equiv\left(\begin{array}{ll}1 & 0\end{array}\right)^{T}$ and $|L\rangle \equiv\left(\begin{array}{ll}0 & 1\end{array}\right)^{T}$ where the superscript $T$ refers to matrix transpose. The density matrix thus assumes the following matrix representation:

$$
\rho=\left(\begin{array}{cc}
\cos ^{2}(\theta / 2) & \cos (\theta / 2) \sin (\theta / 2) \exp (-i \varphi) \\
\cos (\theta / 2) \sin (\theta / 2) \exp (i \varphi) & \sin ^{2}(\theta / 2)
\end{array}\right) .
$$

For density matrices, the conservation of probability in Eq. (3) is expressed as $\operatorname{tr}(\rho)=1$, where $\operatorname{tr}(\rho)=\sum_{i} \rho_{i i}$ is the trace. For the density matrix in our example this becomes

$$
\operatorname{tr}(\rho)=\cos ^{2}(\theta / 2)+\sin ^{2}(\theta / 2)=1 .
$$

This is a physical requirement for any density matrix. However, one can conclude whether it corresponds to a pure or a mixed state by computing the purity:

$$
\begin{aligned}
\operatorname{tr}\left(\rho^{2}\right) & =\operatorname{tr}\left(\sum_{m} \sum_{n} c_{m} c_{n}\left|\psi_{m}\right\rangle\left\langle\psi_{m}|| \psi_{n}\right\rangle\left\langle\psi_{n}\right|\right) \\
& =\sum_{m} \sum_{n} c_{m} c_{n}\left|\left\langle\psi_{m} \mid \psi_{n}\right\rangle\right|^{2} .
\end{aligned}
$$

It follows that $\left|\left\langle\psi_{m} \mid \psi_{n}\right\rangle\right|^{2} \leq 1$; this is because the probability of measuring a state $\left|\psi_{m}\right\rangle$ given that we prepared $\left|\psi_{n}\right\rangle$ is in general less than 1, except when $\left|\psi_{m}\right\rangle=\left|\psi_{n}\right\rangle$, in which case the overlap is 1 . We can then bound Eq. (8) by

$$
0 \leq \sum_{m} \sum_{n} c_{m} c_{n}\left|\left\langle\psi_{m} \mid \psi_{n}\right\rangle\right|^{2} \leq \sum_{m} c_{m} \sum_{n} c_{n}=1,
$$

where we have used the fact that $\sum_{m} c_{m}=1$. One then arrives at the following criterion for purity:

$$
\left\{\begin{array}{l}
\operatorname{tr}\left(\rho^{2}\right)=1 \text { for } c_{m}=c_{n}=1 \Rightarrow \rho \text { is pure } \\
0 \leq \operatorname{tr}\left(\rho^{2}\right)<1 \text { for } c_{m}, c_{n}<1 \Rightarrow \rho \text { is mixed. }
\end{array}\right.
$$

The objective of a QST is to reconstruct the density matrix of an arbitrary state using an appropriate set of measurements, regardless of whether the state is pure or mixed $[9,30,53,128]$. It is this broad applicability that makes QSTs such a powerful tool in quantum information and communication. The procedure behind a QST for two-dimensional quantum states consists of locating the qubit state on the Poincaré sphere; that is, as mentioned before, characterizing the rotation angles about the $x, y$, and $z$ axes. Given that the motion of our qubit state is restricted to rotations on the Poincare sphere, the density matrix can thus be expressed in terms of rotation operators about the $x, y$, and $z$ axes. In two dimensions, these rotation operators are the Pauli matrices, $\sigma_{i}$, plus the identity

$$
\sigma_{1}=\left(\begin{array}{cc}
0 & 1 \\
1 & 0
\end{array}\right) ; \sigma_{2}=\left(\begin{array}{cc}
0 & -i \\
i & 0
\end{array}\right) ; \sigma_{3}=\left(\begin{array}{cc}
1 & 0 \\
0 & -1
\end{array}\right) ; \mathcal{I}=\left(\begin{array}{cc}
1 & 0 \\
0 & 1
\end{array}\right)
$$

The Pauli matrices are traceless operators $\left(\operatorname{tr}\left(\sigma_{i}\right)=0\right)$ that obey the following trace relations:

$$
\operatorname{tr}\left(\sigma_{i} \sigma_{j}\right)=2 \delta_{i, j}, \quad \text { for } i, j=1,2 \text { or } 3 .
$$

This is an expression of the completeness relation between the matrices. Unlike the other Pauli matrices, the identity operator is not traceless, but obeys the trace relations 


$$
\mathcal{I}=\sigma_{0}=\left(\begin{array}{cc}
1 & 0 \\
0 & 1
\end{array}\right) ; \quad \operatorname{tr}\left(\sigma_{0} \sigma_{0}\right)=2 ; \quad \operatorname{tr}\left(\sigma_{0} \sigma_{i}\right)=\operatorname{tr}\left(\sigma_{i} \sigma_{0}\right)=\operatorname{tr}\left(\sigma_{j}\right)=0
$$

That the $\sigma_{i}$ matrices form a complete set means that one can express the density matrix $\rho$ as a linear combination of our $\sigma_{i}$ matrices,

$$
\rho=\frac{1}{2} \sum_{n=0}^{3} \rho_{n} \sigma_{n}
$$

where $\rho_{n}$ are the expectation values of the matrices $\sigma_{n}$ and are obtained from

$$
\operatorname{tr}\left(\rho \sigma_{n}\right)=\frac{1}{2} \operatorname{tr}\left(\sum_{m=0}^{3} \rho_{m} \sigma_{m} \sigma_{n}\right)=\frac{1}{2} \sum_{m=0}^{3} \rho_{m} \operatorname{tr}\left(\sigma_{m} \sigma_{n}\right)=\sum_{m=0}^{3} \rho_{m} \delta_{m, n}=\rho_{n} .
$$

Therefore, provided one can obtain the expectation values, $\rho_{n}$, it is then trivial to reconstruct the density matrix of the system. However, one first needs to clarify the measurement procedure that leads to the computation of $\rho_{n}$. To do so, it is useful to express the matrices $\sigma_{n}$ in terms of eigenvalues and eigenvectors that, as we will show, correspond to physical states that can be measured directly.

The matrices $\sigma_{n}$ each have two eigenvectors, $\left|\lambda_{n}^{0}\right\rangle$ and $\left|\lambda_{n}^{1}\right\rangle$, with eigenvalues $\alpha_{n}^{0}$ and $\alpha_{n}^{1}$ and thus can be expressed as

$$
\sigma_{n}=\alpha_{n}^{0}\left|\lambda_{n}^{0}\right\rangle\left\langle\lambda_{n}^{0}\left|+\alpha_{n}^{1}\right| \lambda_{n}^{1}\right\rangle\left\langle\lambda_{n}^{1}\right| .
$$

Based on this decomposition, we can express the expectation values in Eq. (13) as

$$
\rho_{n}=\operatorname{tr}\left(\rho \sigma_{n}\right)=\alpha_{n}^{0}\left\langle\lambda_{n}^{0}|\rho| \lambda_{n}^{0}\right\rangle+\alpha_{n}^{1}\left\langle\lambda_{n}^{1}|\rho| \lambda_{n}^{1}\right\rangle .
$$

The matrices $\left|\lambda_{n}\right\rangle\left\langle\lambda_{n}\right|$ are called projectors; these are Hermitian and positive operators that form a complete orthonormal set [129]. This is a rather dense description, so let us go through each property one by one.

(1) Hermiticity: Projectors are self-adjoint operators, i.e., they are equal to their own conjugate transpose, and have real expectation values. This is a fundamental requirement for projectors to be physical observables.

(2) Positivity: Positive operators have expectation values greater or equal to 0 . This is a natural requirement for projectors given that their expectation values correspond to probabilities.

(3) Completeness: $\sum_{m}\left|\lambda_{n}^{m}\right\rangle\left\langle\lambda_{n}^{m}\right|=\sigma_{0}$. This is a requirement for conservation of probability

(4) Orthonormality: $\left|\lambda_{n}^{m}\right\rangle\left\langle\lambda_{n}^{m}|| \lambda_{n}^{l}\right\rangle\left\langle\lambda_{n}^{l}|=| \lambda_{n}^{m}\right\rangle\left\langle\lambda_{n}^{l}\right| \delta_{l, m}$. This is not a physical requirement but implies that the eigenstates of two projectors within a complete set have no overlap.

From their definition, one can easily compute the eigenvalues of the matrices $\sigma_{n}$ and show that they are \pm 1 , as shown in Table 2. Interestingly, the eigenvectors correspond to the polarization states on the surface of the Poincare sphere, previously shown in Fig. 2. We now have the recipe to compute the expectation values of the $\sigma_{n}$ matrices in terms of direct measurement that can be performed on the quantum state, and it is as follows:

$$
\rho_{0}=\operatorname{tr}\left(\rho \sigma_{0}\right)=\langle R|\rho| R\rangle+\langle L|\rho| L\rangle,
$$




$$
\begin{gathered}
\rho_{1}=\operatorname{tr}\left(\rho \sigma_{1}\right)=\langle H|\rho| H\rangle-\langle V|\rho| V\rangle, \\
\rho_{2}=\operatorname{tr}\left(\rho \sigma_{2}\right)=\langle D|\rho| D\rangle-\langle A|\rho| A\rangle, \\
\rho_{3}=\operatorname{tr}\left(\rho \sigma_{3}\right)=\langle R|\rho| R\rangle-\langle L|\rho| L\rangle .
\end{gathered}
$$

Observe, however, that the measurements required to compute $\rho_{0}$ are the same as that of $\rho_{3}$. In fact, $\operatorname{tr}\left(\rho \sigma_{0}\right)$ is an expression of the conservation of probability. Given that projectors of a given Pauli matrix form a complete set, the conservation of probability is independent of the measurement basis. Thus, one can deduce that $\rho_{0}$ can be obtained in a similar manner from the projective measurements of $\rho_{1}$ and $\rho_{2}$. Thus, the overcomplete tomographic measurement of a single qubit requires a total of six projective measurements: $d(d+1)$ as mentioned in the introduction, for $d=2$. To illustrate the reconstruction, we will consider two examples: a pure and a mixed qubit states.

- Pure state. Let us consider the state $|\psi\rangle$ expressed in the polarization basis as

$$
|\psi\rangle=\frac{\sqrt{3}}{2}|R\rangle+\frac{1}{2} \exp \left(-i \frac{\pi}{3}\right)|L\rangle
$$

so that the density matrix $\rho=|\psi\rangle\langle\psi|$ reads

$$
\rho=\left(\begin{array}{cc}
\frac{3}{4} & \frac{\sqrt{3}}{4} \exp \left(i \frac{\pi}{3}\right) \\
\frac{\sqrt{3}}{4} \exp \left(-i \frac{\pi}{3}\right) & \frac{1}{4}
\end{array}\right)
$$

We then perform the tomographic measurement of the state by performing the necessary projections, as shown in Table 3 .

Next we compute the expectation values $\rho_{n}$,

$$
\rho_{0}=1 ; \quad \rho_{1}=\frac{\sqrt{3}}{4} ; \quad \rho_{2}=-\frac{3}{4} ; \quad \rho_{3}=\frac{1}{2},
$$

Table 2. Eigenvectors and Eigenvalues of the Identity and Pauli Matrices in the Polarization Basis

\begin{tabular}{lcccc}
\hline Matrices $\sigma_{n}$ & $\alpha_{n}^{0}$ & $\left|\lambda_{n}^{0}\right\rangle$ & $\alpha_{n}^{1}$ & $\left|\lambda_{n}^{1}\right\rangle$ \\
\hline$\sigma_{0}$ & 1 & $|R\rangle \equiv\left(\begin{array}{c}1 \\
0\end{array}\right)$ & 1 & $|L\rangle \equiv\left(\begin{array}{c}0 \\
1\end{array}\right)$ \\
\hline$\sigma_{1}$ & -1 & $|V\rangle \equiv \frac{1}{\sqrt{2}}\left(\begin{array}{c}1 \\
-1\end{array}\right)$ & 1 & $|H\rangle \equiv \frac{1}{\sqrt{2}}\left(\begin{array}{c}1 \\
1\end{array}\right)$ \\
\hline$\sigma_{2}$ & -1 & $|A\rangle \equiv \frac{-i}{\sqrt{2}}\left(\begin{array}{c}1 \\
-i\end{array}\right)$ & 1 & $|D\rangle \equiv \frac{-i}{\sqrt{2}}\left(\begin{array}{c}1 \\
i\end{array}\right)$ \\
\hline$\sigma_{3}$ & 1 & $|R\rangle \equiv\left(\begin{array}{c}1 \\
0\end{array}\right)$ & -1 & $|L\rangle \equiv\left(\begin{array}{c}0 \\
1\end{array}\right)$ \\
\hline
\end{tabular}

Table 3. Tomographic Measurements of a Pure State

\begin{tabular}{lccccc}
\hline$\langle R|\rho| R\rangle$ & $\langle L|\rho| L\rangle$ & $\langle H|\rho| H\rangle$ & $\langle V|\rho| V\rangle$ & $\langle D|\rho| D\rangle$ & $\langle A|\rho| A\rangle$ \\
\hline $3 / 4$ & $1 / 4$ & $\frac{4+\sqrt{3}}{8}$ & $\frac{4-\sqrt{3}}{8}$ & $1 / 8$ & $7 / 8$ \\
\hline
\end{tabular}


and reconstruct the density matrix:

$$
\begin{aligned}
\rho & =\frac{1}{2}\left(\begin{array}{ll}
1 & 0 \\
0 & 1
\end{array}\right)+\frac{\sqrt{3}}{8}\left(\begin{array}{ll}
0 & 1 \\
1 & 0
\end{array}\right)-\frac{3}{8}\left(\begin{array}{cc}
0 & -i \\
i & 0
\end{array}\right)+\frac{1}{4}\left(\begin{array}{cc}
1 & 0 \\
0 & -1
\end{array}\right) \\
& =\left(\begin{array}{cc}
\frac{3}{4} & \frac{\sqrt{3}+3 i}{8} \\
\frac{\sqrt{3}-3 i}{8} & \frac{1}{4}
\end{array}\right)=\left(\begin{array}{cc}
\frac{3}{4} & \frac{\sqrt{3}}{4} \exp \left(i \frac{\pi}{3}\right) \\
\frac{\sqrt{3}}{4} \exp \left(-i \frac{\pi}{3}\right) & \frac{1}{4}
\end{array}\right) .
\end{aligned}
$$

- Mixed state. Now we consider the case of a mixed state $\rho$ expressed in the polarization basis as follows:

$$
\rho=\frac{1}{3}|R\rangle\left\langle R\left|+\frac{2}{3}\right| L\right\rangle\langle L|=\left(\begin{array}{cc}
\frac{1}{3} & 0 \\
0 & \frac{2}{3}
\end{array}\right)
$$

Similarly, we perform a tomographic measurement of the state obtaining the projections shown in Table 4.

We then compute the expectation values $\rho_{n}$,

$$
\rho_{0}=1 ; \quad \rho_{1}=0 ; \quad \rho_{2}=0 ; \quad \rho_{3}=-\frac{1}{3},
$$

and reconstruct the density matrix:

$$
\rho=\frac{1}{2}\left(\begin{array}{ll}
1 & 0 \\
0 & 1
\end{array}\right)-\frac{1}{6}\left(\begin{array}{cc}
1 & 0 \\
0 & -1
\end{array}\right)=\left(\begin{array}{cc}
\frac{1}{3} & 0 \\
0 & \frac{2}{3}
\end{array}\right)
$$

A graphical representation of the density matrix in terms of real and imaginary parts is presented in Figs. 4(a) and 4(b) for the pure state $|\psi\rangle=\frac{\sqrt{3}}{2}|R\rangle+\frac{1}{2} \exp \left(-i \frac{\pi}{3}\right)|L\rangle$ and the mixed state $\rho=\frac{1}{3}|R\rangle\left\langle R\left|+\frac{2}{3}\right| L\right\rangle\langle L|$, respectively.

A useful way to visualize these measurements is to see that they are made up of projections into two orthogonal bases, say $|L\rangle$ and $|R\rangle$, and into four mutually unbiased bases (MUBs): $|H\rangle,|V\rangle,|A\rangle$, and $|D\rangle$. The MUBs can be constructed from superpositions of the two orthogonal bases, i.e., the horizontal MUB, $|H\rangle$, can be written as a superposition of $|L\rangle$ and $|R\rangle$. Although obvious for polarization, we show them graphically in Fig. 5 as we will build up this figure throughout the tutorial with examples beyond polarization. The mutually unbiased states have the property that the overlap with one of the orthogonal states always yields an outcome with a probability of $1 / d$, where $d$ is the dimension of the Hilbert space. For polarization $d=2$, so this is $1 / 2$.

\subsection{Spatial Mode Qubit}

While in the above we have presented the qubit QST in terms of polarization, one should note that the choice of degree of freedom is arbitrary. An alternative and topical degree of freedom is the spatial mode of the photon [130]. The term spatial mode refers to transverse solutions of the wave equation in the paraxial limit; that is, when the variation in transverse momentum is negligible in comparison to its longitudinal counterpart. In this regime, a family of solutions arise: Hermite-Gaussian, LaguerreGaussian, and Bessel-Gaussian modes, just to name a few. Some of these modes carry

Table 4. Tomographic Measurements of a Mixed State

\begin{tabular}{lccccc}
\hline$\langle R|\rho| R\rangle$ & $\langle L|\rho| L\rangle$ & $\langle H|\rho| H\rangle$ & $\langle V|\rho| V\rangle$ & $\langle D|\rho| D\rangle$ & $\langle A|\rho| A\rangle$ \\
\hline $1 / 3$ & $2 / 3$ & $1 / 2$ & $1 / 2$ & $1 / 2$ & $1 / 2$ \\
\hline
\end{tabular}


discrete units of a fundamental quantum number: the OAM. Conserved at the single photon level [67], the OAM degree of freedom is particularly attractive in classical and quantum information, communication, and computation [131-135]. Unlike polarization, the state space of OAM modes is infinitely large, allowing more information to be encoded in photons [75,76,79,136-139].

Recall the polarization qubit state in Eq. (2). An analogous description can be provided in terms of spatial modes that carry OAM [140]:

$$
|\psi\rangle=\cos (\theta / 2)\left|\ell_{1}\right\rangle+\exp (i \varphi) \sin (\theta / 2)\left|\ell_{2}\right\rangle,
$$

where the ket $|\ell\rangle$ refers to a paraxial field that carries $\ell \hbar$ units of OAM. Such a field can be expressed in cylindrical coordinates $(r, \phi, z)$ [141]:

$$
|\ell\rangle \equiv A(r, z) \exp (i \ell \phi),
$$

where $A(r, z)$ is an amplitude term that varies transversally and longitudinally. The intensity and phase of some OAM modes of the Laguerre-Gaussian type are shown

\section{Figure 4}

(a)
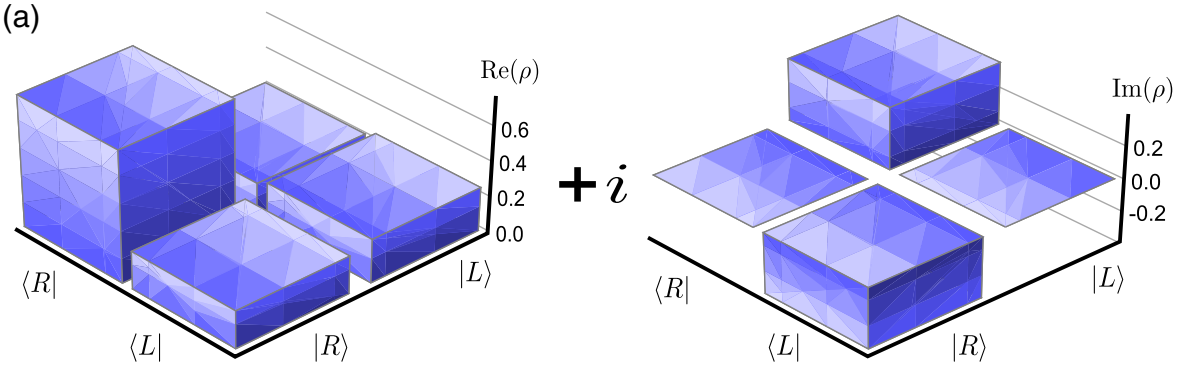

(b)
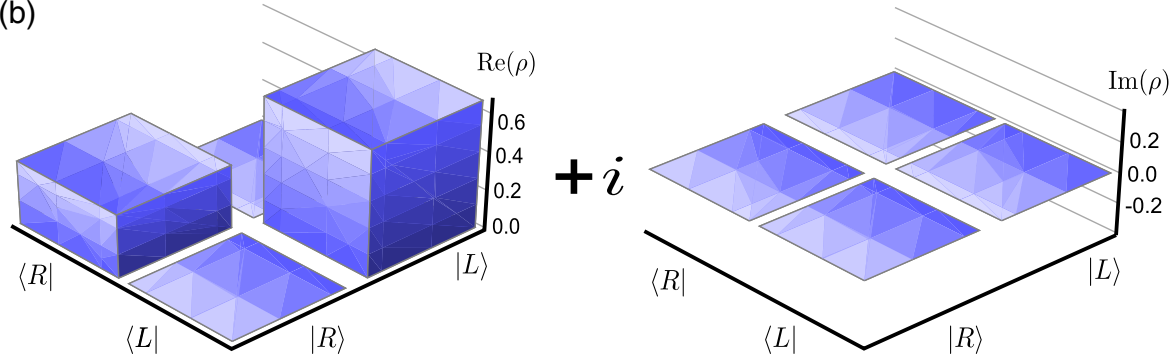

Graphical representation of the density matrix in terms of its real and imaginary components for the (a) pure state and (b) mixed state examples as given in the main body text.

\section{Figure 5}

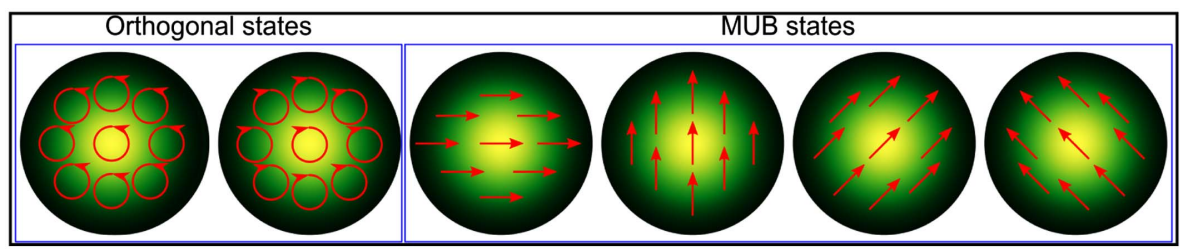

Graphical representation of orthogonal and mutually unbiased states used in the QST projections. Here only the polarization matters, shown overlaid on a Gaussian mode. 
in Fig. 6. The azimuthal phase creates a twisted wavefront with a central discontinuity where the phase is undefined, resulting in an intensity null.

Observe that the field of the OAM mode is separable in both amplitude and azimuthal phase, $\arg [\exp (i \ell \phi)]$; that is, the amplitude and azimuthal phase can

\section{Figure 6}

(a)
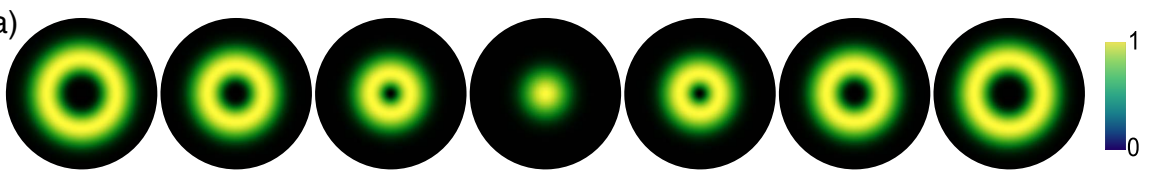

(b)
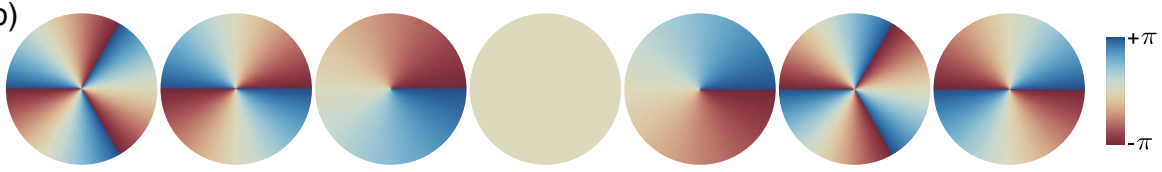

(a) Intensities and (b) phase maps of OAM modes carrying, from left to right, $\ell=-3,-2, \ldots,+2$, and +3 units of OAM.

\section{Figure 7}

(a)

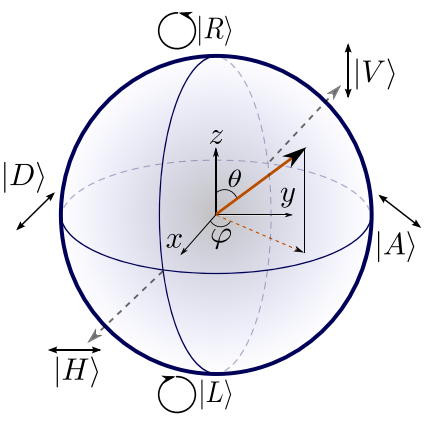

(c)

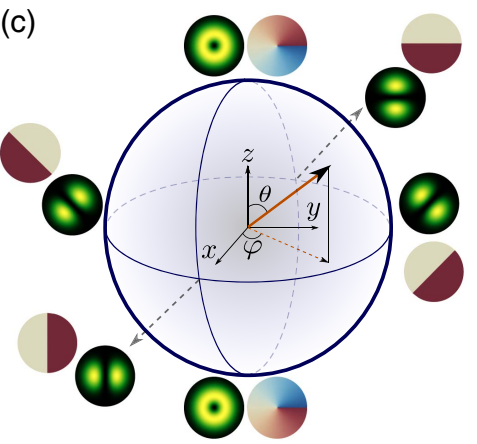

(b)

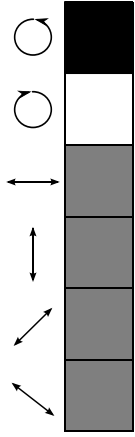

(d)
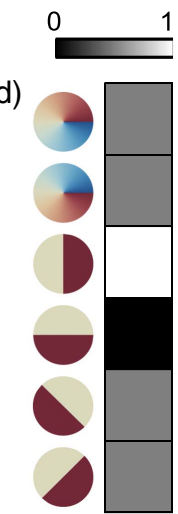

(a) Representation of polarization states on the Poincaré sphere. The poles represent the eigenstates of the basis, from which all other states are constructed. An arbitrary polarization state thus maps to a point on the surface of the Poincare sphere. (b) The normalized outcomes of projective measurements onto the eigenstates of the Pauli matrices, for given horizontally polarized state. (c) Equivalently, one can construct a similar sphere, a Bloch sphere, where the poles correspond to OAM eigenstates. Here, the eigenstates of the Pauli matrices correspond to spatial modes. (d) From projective measurement onto these spatial modes, one performs the quantum-state tomographic measurement of an OAM state $(|\ell\rangle+|-\ell\rangle) / \sqrt{2}$ with $\ell=-1$. 
be factorized. The orthogonality of OAM modes at a given $z$ plane (say $z=z_{0}$ ) may be expressed by

$$
\left\langle\ell_{1} \mid \ell_{2}\right\rangle=\int_{0}^{\infty} \mathrm{d} r r A\left(r, z_{0}\right) \int_{0}^{2 \pi} \mathrm{d} \phi \exp \left(i\left(\ell_{2}-\ell_{1}\right) \phi\right)=\delta_{\ell_{1}, \ell_{2}} 2 \pi \int_{0}^{\infty} \mathrm{d} r r A\left(r, z_{0}\right) .
$$

Similar to polarization, OAM qubits can be represented on the surface of a sphere, the OAM Bloch sphere [140], as shown in Fig. 7. Here the poles are the OAM states, while the equator represents the Hermite-Gaussian modes, as detailed in Fig. 8, for $\ell_{1}=-\ell_{2}=1$. Observe that the superposition of two oppositely charged OAM states leads to azimuthal fringes, which then rotate with the intermodal phase $\varphi$. This is similar to the rotation of the linear polarization states in Fig. 2.

A QST of an OAM qubit follows the same procedure as that outlined previously. The density matrix is expanded in terms of Pauli matrices and the identity. The eigenvectors now take on a different meaning: rather than polarization states, they now correspond to OAM modes and their superpositions, as shown in Fig. 9. Means to perform projective measurements on these spatial modes have been extensively reported for quantum [19,142] and classical light [143-147]. Progress in liquid crystal technology and digital micro mirror devices has made it possible to generate and detect arbitrary spatial modes using digital holograms (see Ref. [130] and Ref. [148] for

\section{Figure 8}

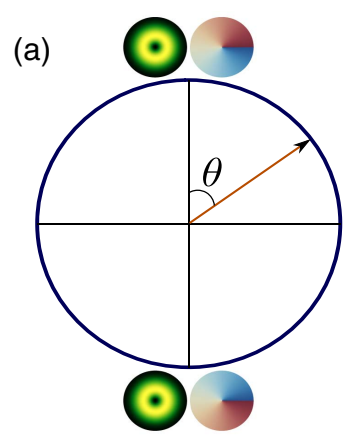

(b)
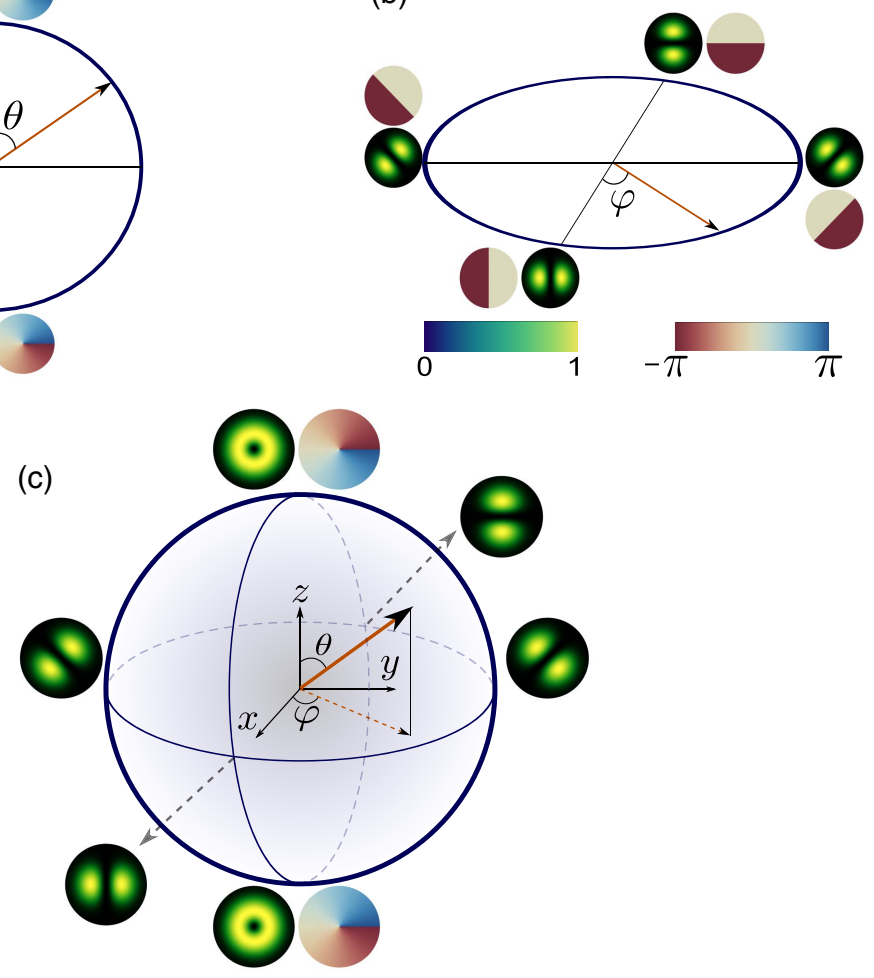

Bloch sphere description of an OAM qubit in Eq. (23) with $\ell_{1}=-\ell_{2}=1$. Control over the amplitude parameter $\theta$ and phase parameter $\varphi$, as shown in (a) and (b), respectively, leads to (c) a description of the general OAM qubit on a unit sphere where the poles are the pure OAM states. 
a comprehensive review and tutorial, respectively). This has opened avenues for an all-digital realization of QST with spatial modes. The core idea is to consider the pattern creation step but in reverse. If a particular hologram were to convert a Gaussian mode into a desired pattern, then in reverse the same hologram will convert the pattern into a Gaussian. As Gaussians are the only modes that couple into single-mode fiber (SMF), we have the means of a "pattern sensitive" detector, as illustrated in Fig. 10. An example of projective measurements, together with the reconstructed density matrix of pure OAM qubit state $|\psi\rangle=(|1\rangle+|-1\rangle) \sqrt{2}$, is shown in Fig. 11 .

Note again that we can see the link between orthogonal and MUB projections, this time into $|\ell\rangle$ and $|-\ell\rangle$ states, plus the four MUBs made up of superpositions of these: $|\ell\rangle+\exp (i \theta)|-\ell\rangle$ for $\theta=[0, \pi / 2, \pi, 3 \pi / 2]$, as shown graphically in Fig. 12. Because such MUBs require amplitude modulation to implement, one often approximates them in the projective measurement as $\arg [|\ell\rangle+\exp (i \theta)|-\ell\rangle]$, producing a binary phase pattern rather than an amplitude function. This is why all the Pauli matrices in Fig. 9 are phase-only patterns. In general the OAM example can be replaced with arbitrary modes by substituting $|\ell\rangle \rightarrow\left|M_{1}\right\rangle$ and $|-\ell\rangle \rightarrow\left|M_{2}\right\rangle$ everywhere in the above analysis.

\section{Figure 9}

\begin{tabular}{|c|c|c|c|c|}
\hline Matrices $\sigma_{n}$ & $\alpha_{n}^{0}$ & $\left|\lambda_{n}^{0}\right\rangle$ & $\alpha_{n}^{1}$ & $\left|\lambda_{n}^{1}\right\rangle$ \\
\hline$\sigma_{0}$ & 1 & & 1 & \\
\hline$\sigma_{1}$ & -1 & & 1 & \\
\hline$\sigma_{2}$ & -1 & & 1 & \\
\hline$\sigma_{3}$ & -1 & & 1 & \\
\hline
\end{tabular}

Eigenvectors and eigenvalues of the identity and Pauli matrices in the OAM basis.

\section{Figure 10}

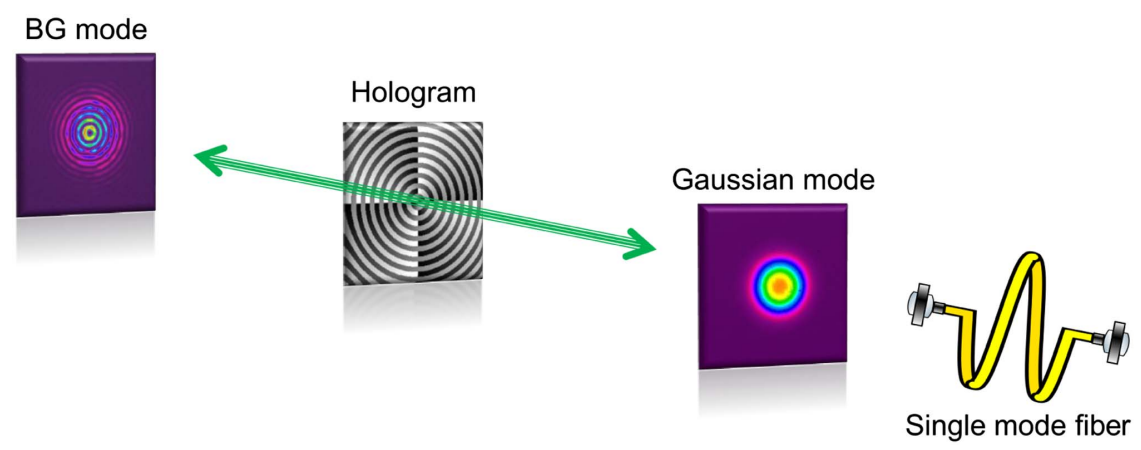

Detection of a spatial mode requires a "pattern sensitive" detector. This is achieved by exploiting the reciprocity of light, passing the incoming beam backward through the hologram that would detect it. 


\subsection{Biphoton Qubits}

Transitioning from single particles to multi-particle systems allows for the existence of correlations, one of the most remarkable being non-local entanglement [149]. Mathematically, the states of entangled systems are non-separable, i.e., the states in an entangled system do not factorize into product states. Physically this means that, through non-local correlations, the unknown state of one particle can be uniquely determined through measurements on its entangled partner(s). Entanglement is a valuable resource in quantum information and quantum computation, and as such requires certification. While there exists various means to characterize entanglement, a standard and widely used approach is to first reconstruct the density matrix of the state under study and then determine whether it is entangled or not. The tool of choice for this task is a QST, first performed on OAM modes with physical holograms [67] and later with digital holograms [142].

Here we look at a two-qubit system $(N=2$ and $d=2)$ and go through the method behind a two-particle QST. As discussed before, the choice of degree of freedom is

\section{Figure 11}

(a)

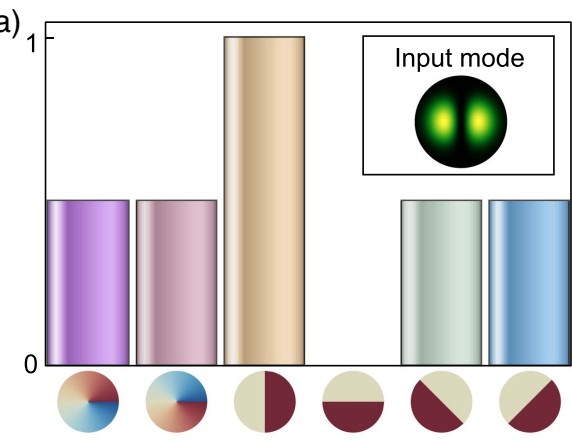

(b)

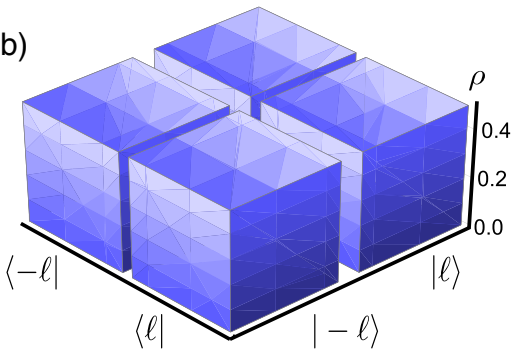

(a) Graphical representation of the outcomes of the projective measurements of a QST on the state $|\psi\rangle=(|1\rangle+|-1\rangle) / \sqrt{2}$, shown in the inset. (b) Based on these tomographic measurements, one reconstructs the density matrix of the state.

\section{Figure 12}

(a) Orthogonal states MUB states

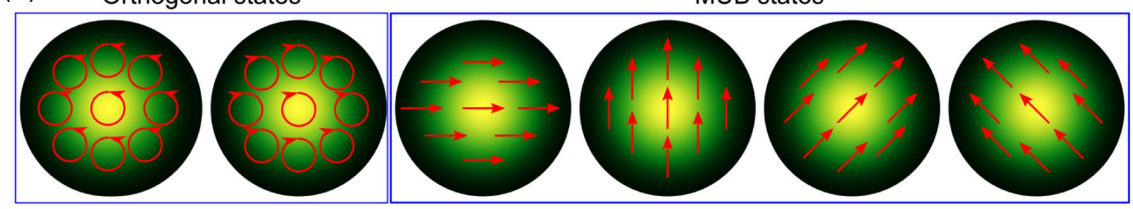

(b)

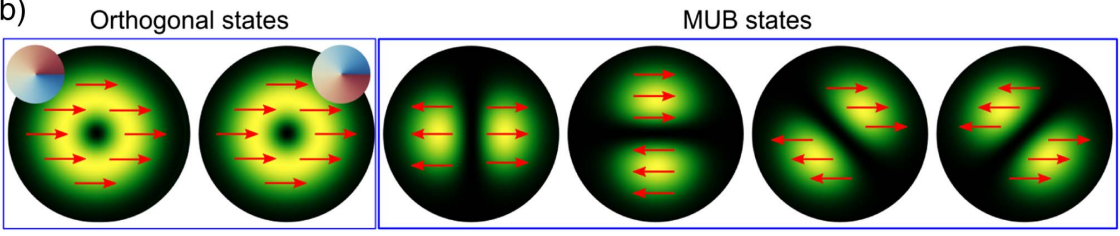

Graphical representation of the orthogonal and MUBs used in the QST projections for (a) polarization and (b) OAM modes. In the case of the latter, the polarization is no longer important and so is shown as horizontal everywhere. The patterns are shown as intensity functions, while the actual projections are often done with phase-only approximations to these. 
arbitrary and as such we will choose the OAM degree of freedom to illustrate the tomographic measurements. We will work in a generic OAM subspace with eigenstates $|-\ell\rangle$ and $|\ell\rangle$ (the same substitution rules apply as mentioned earlier should the reader wish to adapt the basis to another mode type)

We start by describing a general two-particle system with a density matrix $\rho$ as

$$
\rho=\sum_{m} c_{m}\left|\psi_{m}\right\rangle\left\langle\psi_{m}\right|
$$

where the pure states $\left|\psi_{m}\right\rangle$ are now two particle qubit states, described by

$$
\left|\psi_{m}\right\rangle=\sum_{i, j} \alpha_{m}^{i j}|i\rangle_{A} \otimes|j\rangle_{B}
$$

The states $|i\rangle$ and $|j\rangle$ are eigenstates of the systems $A$ and $B$, respectively, with associated complex coefficients $\alpha_{m}^{i j}$ that define the $m$ th state $\left|\psi_{m}\right\rangle$. The symbol $\otimes$ is a tensor product operation that, in essence, is a way to multiply two state spaces of dimensions $d_{1}$ and $d_{2}$, respectively, to form a new larger state space with dimension $d_{1} \times d_{2}$. In the case of a two-qubit state, the joint system is four dimensional. It is worth spending some time describing the computation of the tensor product operation.

Given two matrices $S$ and $T$ (we will assume without loss of generality that these are square matrices), the tensor product $S \otimes T$ produces a new matrix $M$ computed by

$$
M=S \otimes T=\left(\begin{array}{ccc}
S_{1,1} \times T & \cdots & S_{1, N} \times T \\
\vdots & \ddots & \vdots \\
S_{N, 1} \times T & \cdots & S_{N, N} \times T
\end{array}\right) .
$$

Using this procedure we can deduce the basis vectors for the two-qubit states of interest, $\left|\psi_{m}\right\rangle$, by considering all the tensor product combinations $|i\rangle \otimes|j\rangle$. In the spatial mode basis of interest here, these are the OAM eigenstates $| \pm \ell\rangle$. We then obtain the basis states for the joint system as follows:

$$
\begin{aligned}
& |\ell\rangle \otimes|\ell\rangle=\left(\begin{array}{l}
1 \\
0
\end{array}\right) \otimes\left(\begin{array}{l}
1 \\
0
\end{array}\right)=\left(\begin{array}{l}
1\left(\begin{array}{l}
1 \\
0
\end{array}\right) \\
0\left(\begin{array}{l}
1 \\
0
\end{array}\right)
\end{array}\right)=\left(\begin{array}{l}
1 \\
0 \\
0 \\
0
\end{array}\right), \\
& |\ell\rangle \otimes|-\ell\rangle=\left(\begin{array}{l}
1 \\
0
\end{array}\right) \otimes\left(\begin{array}{l}
0 \\
1
\end{array}\right)=\left(\begin{array}{l}
1\left(\begin{array}{l}
0 \\
1
\end{array}\right) \\
0\left(\begin{array}{l}
0 \\
1
\end{array}\right)
\end{array}\right)=\left(\begin{array}{l}
0 \\
1 \\
0 \\
0
\end{array}\right), \\
& |-\ell\rangle \otimes|\ell\rangle=\left(\begin{array}{l}
0 \\
1
\end{array}\right) \otimes\left(\begin{array}{l}
1 \\
0
\end{array}\right)=\left(\begin{array}{l}
0\left(\begin{array}{l}
1 \\
0
\end{array}\right) \\
1\left(\begin{array}{l}
1 \\
0
\end{array}\right)
\end{array}\right)=\left(\begin{array}{l}
0 \\
0 \\
1 \\
0
\end{array}\right),
\end{aligned}
$$




$$
|-\ell\rangle \otimes|-\ell\rangle=\left(\begin{array}{l}
0 \\
1
\end{array}\right) \otimes\left(\begin{array}{l}
0 \\
1
\end{array}\right)=\left(\begin{array}{l}
0\left(\begin{array}{l}
0 \\
1
\end{array}\right) \\
1\left(\begin{array}{l}
0 \\
1
\end{array}\right)
\end{array}\right)=\left(\begin{array}{l}
0 \\
0 \\
0 \\
1
\end{array}\right)
$$

Now that we have an understanding of the tensor product operation, the state-tomography measurement of the two-qubit state can be intuitively understood. The state of each qubit can be characterized with the set of rotation matrices (Pauli matrices) plus the identity matrix. The joint state, therefore, follows a tensor product construction as follows:

$$
\rho=\left(\frac{1}{2} \sum_{m=0}^{3} \rho_{m} \sigma_{m}\right)_{A} \otimes\left(\frac{1}{2} \sum_{n=0}^{3} \rho_{n} \sigma_{n}\right)_{B}=\frac{1}{4} \sum_{m, n=0}^{3} \rho_{m n} \sigma_{m} \otimes \sigma_{n} .
$$

The above construction naturally leads to the description of the qubit pair on the surface of a higher order Bloch sphere, as shown in Fig. 13. From Eq. (33), the state of the qubit pair is defined by a set of single-qubit rotations, together with single-qubit identity operators. Similar to the case of a single qubit, these rotations occur on the surface of a sphere, a higher order Bloch sphere. In this description, the states on the sphere follow the same tensor product construction as that in Eq. (27), as shown in Fig. 13. In the case of OAM states, the poles correspond to the tensor product of single-qubit OAM states. While there are many tensor product combinations of single-photon qubit states, there is only one rule for constructing the higher order Bloch sphere: each single qubit must have orthogonal states on the poles of the sphere. For example, one cannot construct a higher order Bloch sphere with the states $|\ell\rangle|-\ell\rangle$ and $|\ell\rangle|\ell\rangle$ on the poles. This is because any two-qubit pair formed as a linear superposition of these two-qubit states factorizes with respect to each subsystem. This simply means that one can write the two-qubit state as $|\psi\rangle=(\cdot)_{A} \otimes(\cdot)_{B}$. This can be shown as follows:

\section{Figure 13}

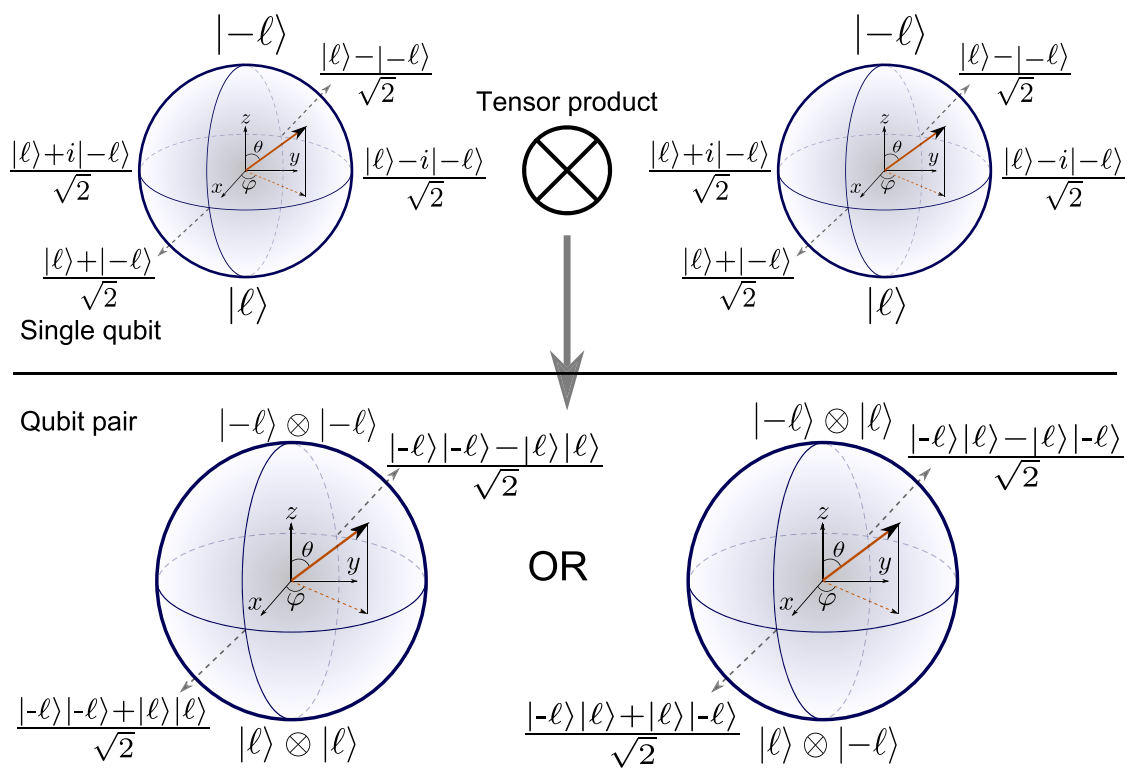

Description of qubit pairs on the higher order Bloch sphere. States on the surface of the higher order Bloch sphere are constructed from the tensor product of qubit states from two Bloch spheres, each describing a subsystem (photon). The entire space is four dimensional, shown here as two-dimensional subspaces for visualization purposes. 


$$
\begin{aligned}
|\psi\rangle & =\cos (\theta / 2)|\ell\rangle_{A}|-\ell\rangle_{B}+\exp (i \varphi) \sin (\theta / 2)|\ell\rangle_{A}|\ell\rangle_{B} \\
\Rightarrow|\psi\rangle & =|\ell\rangle_{A} \otimes(\cos (\theta / 2)|-\ell\rangle+\exp (i \varphi) \sin (\theta / 2)|\ell\rangle)_{B}
\end{aligned}
$$

Observe that the two-qubit state is parameterized by $\theta$ and $\varphi$, and can be entirely characterized by a single qubit rotation on subsystem B alone. We thus say that the two subsystems factorize, or equivalently, that they are separable. The notion of separability will be treated in more detail a little later.

Note that an arbitrary state on the surface of the higher order Bloch sphere cannot be described by a single qubit rotation on one subsystem alone. In other words, the higher order Bloch sphere describes a set of both separable and non-separable states. In the case of two-qubit states, this non-separability is what we traditionally refer to as "quantum entanglement." In general, arbitrary states on the higher order Bloch spheres in Fig. 13 are represented as

$$
|\psi\rangle=\cos (\theta / 2)\left|\ell_{1}\right\rangle\left|\ell_{2}\right\rangle+\exp (i \varphi) \sin (\theta / 2)\left|-\ell_{1}\right\rangle\left|-\ell_{2}\right\rangle \neq(\cdot)_{A} \otimes(\cdot)_{B} .
$$

Fortunately, the separability of the state does not affect the reconstruction procedure through QST. In what follows, we will outline the steps to perform a two-qubit QST for an arbitrary state represented by the density matrix, $\rho$.

Once again, the task of a QST is to realize direct measurements to compute the expectation values $\rho_{m n}$. We follow the same tensor product construction of the expectation value to express $\rho_{m n}$ in terms of eigenvalues and eigenvectors of the basis operators for the joint system; these are $\sigma_{m} \otimes \sigma_{n}$ and can be expressed as follows:

$$
\sigma_{m} \otimes \sigma_{n}=\left(\alpha_{m}^{0}\left|\lambda_{m}^{0}\right\rangle\left\langle\lambda_{m}^{0}\left|+\alpha_{m}^{1}\right| \lambda_{m}^{1}\right\rangle\left\langle\lambda_{m}^{1}\right|\right) \otimes\left(\alpha_{n}^{0}\left|\lambda_{n}^{0}\right\rangle\left\langle\lambda_{n}^{0}\left|+\alpha_{n}^{1}\right| \lambda_{n}^{1}\right\rangle\left\langle\lambda_{n}^{1}\right|\right) .
$$

By expanding the expression above, the measurements required in a QST can be directly read as

$$
\begin{aligned}
\sigma_{m} \otimes \sigma_{n}= & \alpha_{m}^{0} \alpha_{n}^{0}\left|\lambda_{m}^{0}\right\rangle\left\langle\lambda_{m}^{0}|\otimes| \lambda_{n}^{0}\right\rangle\left\langle\lambda_{n}^{0}\left|+\alpha_{m}^{1} \alpha_{n}^{1}\right| \lambda_{m}^{1}\right\rangle\left\langle\lambda_{m}^{1}|\otimes| \lambda_{n}^{1}\right\rangle\left\langle\lambda_{n}^{1}\right| \\
& +\alpha_{m}^{1} \alpha_{n}^{0}\left|\lambda_{m}^{1}\right\rangle\left\langle\lambda_{m}^{1}|\otimes| \lambda_{n}^{0}\right\rangle\left\langle\lambda_{n}^{0}\left|+\alpha_{m}^{0} \alpha_{n}^{1}\right| \lambda_{m}^{0}\right\rangle\left\langle\lambda_{m}^{0}|\otimes| \lambda_{n}^{1}\right\rangle\left\langle\lambda_{n}^{1}\right| .
\end{aligned}
$$

The projectors $\left|\lambda_{m}\right\rangle\left\langle\lambda_{m}\right|$ have been discussed in the previous section and we have shown that they can be realized through direct measurement on the quantum state. In the case of two qubits, the tensor product $\left|\lambda_{m}\right\rangle\left\langle\lambda_{m}|\otimes| \lambda_{n}\right\rangle\left\langle\lambda_{n}\right|$ means that one must perform joint projective measurements on both photons. Practically, this implies that the projective measurements must be done in coincidence. This can be done using single photon detectors and a counting module. The expectation values $\rho_{m n}$ then take the following form:

$$
\begin{aligned}
\rho_{m n}= & \alpha_{m}^{0} \alpha_{n}^{0}\left\langle\lambda_{m}^{0} \lambda_{n}^{0}|\rho| \lambda_{m}^{0} \lambda_{n}^{0}\right\rangle+\alpha_{m}^{1} \alpha_{n}^{1}\left\langle\lambda_{m}^{1} \lambda_{n}^{1}|\rho| \lambda_{m}^{1} \lambda_{n}^{1}\right\rangle \\
& +\alpha_{m}^{1} \alpha_{n}^{0}\left\langle\lambda_{m}^{1} \lambda_{n}^{0}|\rho| \lambda_{m}^{1} \lambda_{n}^{0}\right\rangle+\alpha_{m}^{0} \alpha_{n}^{1}\left\langle\lambda_{m}^{0} \lambda_{n}^{1}|\rho| \lambda_{m}^{0} \lambda_{n}^{1}\right\rangle .
\end{aligned}
$$

In the above, we have used a compact notation for the projective measurement, where $\left|\lambda_{m}^{0} \lambda_{n}^{0}\right\rangle \equiv\left|\lambda_{m}^{0}\right\rangle \otimes\left|\lambda_{n}^{0}\right\rangle$. Note the order of the eigenstate in both the bra and ket in the expression of the expectation value: the first state refers a measurement on particle $\mathrm{A}$, while the second state refers to a measurement on particle B. For example, the expectation value $\left\langle\lambda_{m}^{0} \lambda_{n}^{1}|\rho| \lambda_{m}^{0} \lambda_{n}^{1}\right\rangle$ is obtained by projecting photons $\mathrm{A}$ and $\mathrm{B}$ on the state $\left|\lambda_{m}^{0}\right\rangle$ and $\left|\lambda_{n}^{1}\right\rangle$, respectively, and measuring in coincidence. The eigenvalues $\alpha_{m}$ and eigenvectors $\left|\lambda_{m}\right\rangle$ are known from the single-qubit QST; we can thus write the expression of the expectation values $\rho_{m n}$ in terms of direct projective measurements. With four 
measurements per expectation value, one would in principle perform a total of 64 measurements. However, upon close examination of the expectation values, one realizes that a total of only 36 measurements is necessary. This is because some of the expectation values share a common set of measurements. Recall from single-qubit QST that the projectors of the identity matrix are the same as that of any single Pauli matrix. Therefore, by measuring the expectation value $\rho_{m n}$ for $m, n>0$, one can compute $\rho_{00}, \rho_{m 0}$, and $\rho_{0 n}$. This is what reduces the number of necessary projective measurements from 64 to 36, the value given in the introduction for a biphoton system as $[d(d+1)]^{2}$, which for $d=2$ yields 36 .

Experimental implementation is, however, not without its own set of challenges. Engineering quantum states is a probabilistic process. A standard method of generating two-photon states that are correlated is through spontaneous parametric down conversion (SPDC), where a nonlinear crystal is pumped with photons from a laser, as shown in Fig. 14(a). An entangled pair is produced with a certain probability depending on the type of nonlinear crystal used. This results in fluctuations in photon number measured by the single-photon detectors, resulting in experimental errors that affect the reconstruction of the quantum state. Statistical techniques can be employed to mitigate these errors, one being the maximum likelihood estimation [142]. The principles of the method are as follows:

\section{Figure 14}

(a)

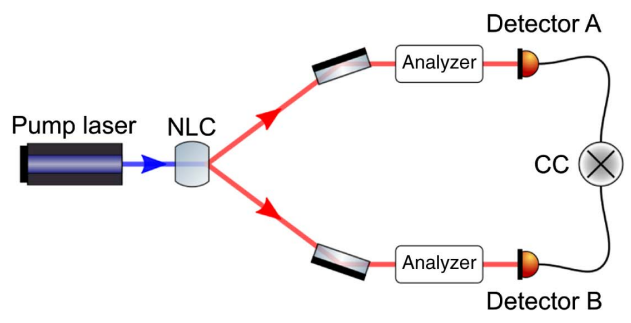

(c)

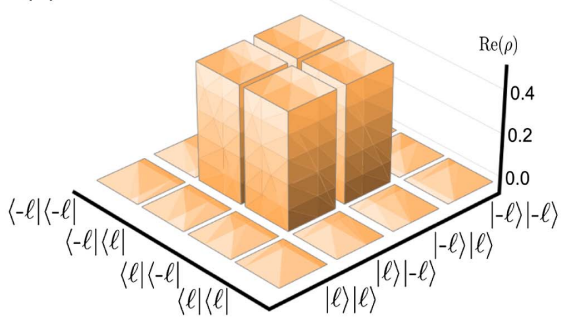

(b)

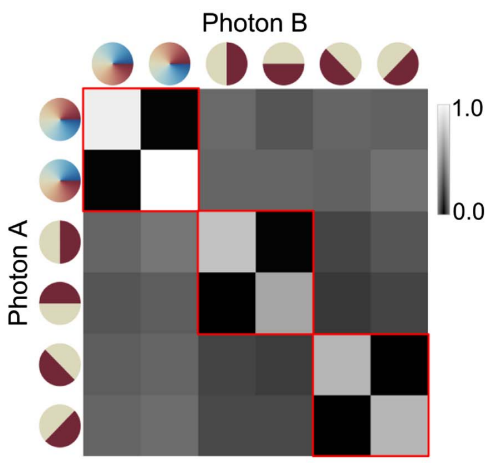

(d)

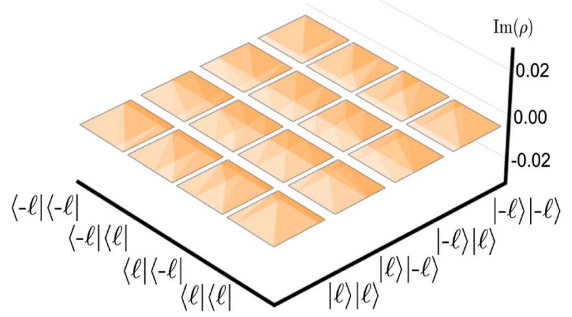

Quantum-state tomographic measurement of a two-photon state. (a) An experimental setup to generate entangled two-photon states through spontaneous parametric downconversion in a nonlinear crystal (NLC). The downconverted photons travel to two OAM analyzers, and their quantum states are measured in coincidence. (b) Shows a two-qubit QST where the projected state of each photon in the entangled pair is indicated by its phase map. The color of each box represents the normalized coincidence counts for a given set of projection on the two-photon state. Using the tomographic data, the density matrix is computed, and its real and imaginary components are shown in (c) and (d). The sum of the measurements enclosed by the red squares defines the expectation value of the identity (probability conservation). 
1. Perform the tomographic measurements on the quantum state.

2. Guess a physical density matrix for the state (Hermitian with unit trace and nonnegative eigenvalues)

3. Computationally perform a tomographic measurement of the state based on the guessed density matrix.

4. Compute the difference between the guessed and experimental tomographic measurements: $\chi^{2}=\left(\sum_{i=1}^{36} \frac{C_{\text {exp }}^{i}-C_{\text {guess }}^{i}}{\sqrt{C_{\text {exp }}^{i}+1}}\right)^{2}$, where the subscript $i$ refers to the $i$ th tomographic projections. The experimental tomographic measurements are labeled $C_{\text {exp }}^{i}$, while those simulated based on the guessed density matrix are labeled $C_{\text {guess }}^{i}$.

5. Update the guessed density matrix such that it minimizes the $\chi^{2}$ difference.

This procedure ensures that the reconstructed density matrices meet the criteria required to be physical; i.e., the density matrix is Hermitian with non-negative eigenvalues. An example of a tomographic measurement of the state of OAM entangled photon pairs is shown in Fig. 14(b). Using the maximum likelihood estimation, one reconstructs the real and imaginary components of the density matrix shown in Figs. 14(c) and 14(d), respectively.

But how to actually perform the QST? A summary of the measurement settings for a biphoton QST and the estimation of a Bell parameter (to be discussed later) is shown in Fig. 15. For QST, a total of 36 projective measurements are required (highlighted

\section{Figure 15}

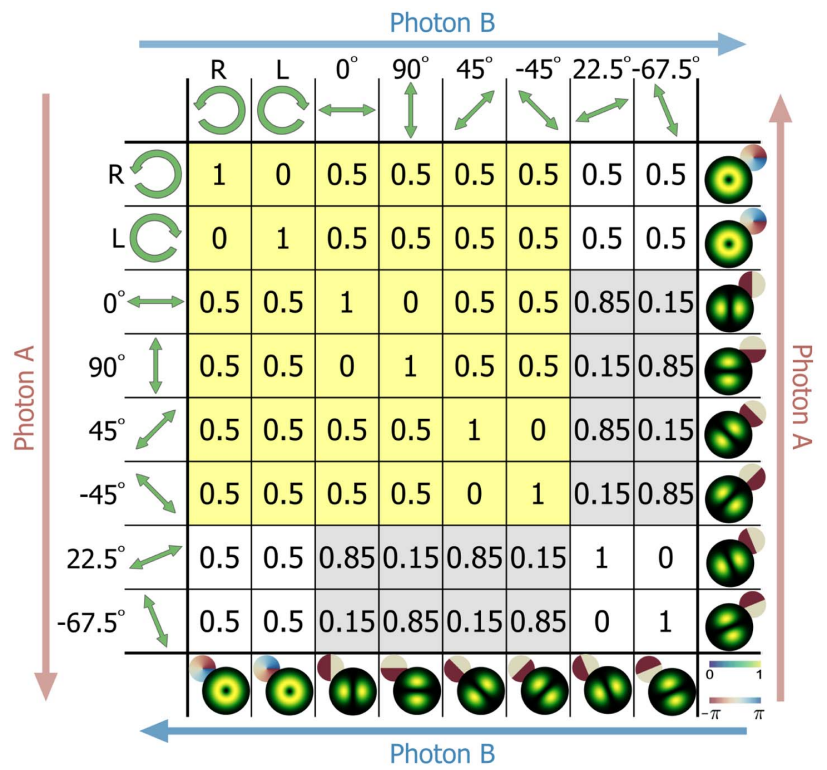

Concept diagram illustrating the measurements required on two photons, $\mathrm{A}$ and $\mathrm{B}$, to perform an over-complete QST (highlighted in yellow) and a Bell violation measurement (highlighted in gray) for polarization and spatial mode correlations. The values show the expected outcomes for a biphoton maximally entangled state. By following the rows/columns through from one end to the other, one finds the equivalent measurement in the alternative basis, i.e., from polarization to spatial mode and vice versa. Measurements on hybrid states can be deduced by selecting a row of one degree of freedom and a column of another, i.e., photon A from the polarization and photon B from the OAM space. The spatial modes are shown with their phases as insets, the latter actually used in the measurement process. 
yellow). The rows and columns in Fig. 15 are labeled with the eigenstates of the degree of freedom onto which the projections are performed; these can, for example, be the polarization or OAM of two entangled photons, or a hybrid polarization-OAM combination (one photon from each degree of freedom). Similarly, the highlighted gray areas show the required measurements for which the Bell parameter is maximized. These are the optimum measurements used to show possible violation of the Clauser-Horne-Shimony-Holt (CHSH) inequality (see later). To translate this to spatial modes, say OAM, one follows the same approach as one would with polarization but with polarization measurements replaced by spatial mode measurements, as illustrated in Fig. 15 (for $|\ell|= \pm 1$ ), with the relation to an actual experiment shown in Fig. 16 (for $|\ell|= \pm 3$ ). As with polarization, 36 measurements are needed, consisting of holograms that depict the two orthogonal modes as well as the four mutually unbiased modes (superpositions of the orthogonal modes), for photon A and B, displayed on holograms A and B. A QST on OAM modes is shown in Fig. 17, with varying degrees of entanglement. It is also possible to mix two degrees of freedom, to form so-called hybrid entangled states [94,104,150,151]. An example of a maximally entangled hybrid state in polarization and spatial (OAM) modes may be expressed as

$$
|\psi\rangle=\frac{1}{\sqrt{2}}\left(|\ell\rangle_{A}|R\rangle_{B}+|-\ell\rangle_{A}|L\rangle_{B}\right)
$$

where we have labeled the states as photon A and photon B. The above state can be described as a point on the surface of a new sphere, the high-order Poincare sphere

\section{Figure 16}
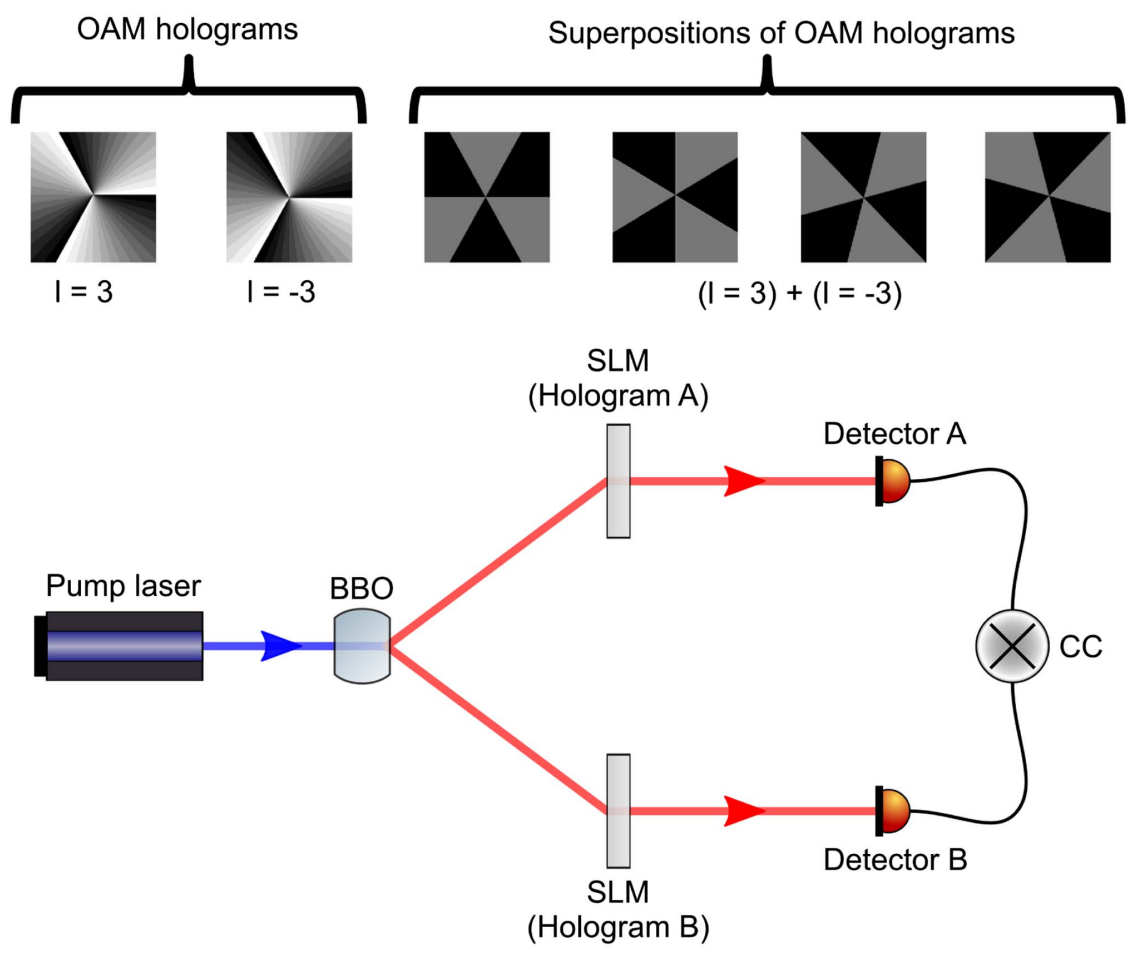

Each entangled photon in the pair is directed to a SLM that displays a hologram. The hologram is programmed with appropriate phase patterns to detect spatial modes. In this example, the holograms are shown for OAM modes of $|\ell|= \pm 3$ as well as the superpositions thereof, all six required for over-complete tomographic reconstruction. Running through six holograms on each SLM produces the 36 measurements required. 
(HOPS) [123-125]. The sphere is constructed from the tensor product of the eigenstates of the individual subsystems, as shown in Fig. 18.

We can express the density matrix of this system analogously to before as

$$
\rho=\left(\frac{1}{2} \sum_{m=0}^{3} \rho_{m} \sigma_{m}\right)^{\text {orbit }} \otimes\left(\frac{1}{2} \sum_{n=0}^{3} \rho_{n} \sigma_{n}\right)^{\text {spin }}=\frac{1}{4} \sum_{m, n=0}^{3} \rho_{m n} \sigma_{m}^{\text {orbit }} \otimes \sigma_{n}^{\text {spin }},
$$

\section{Figure 17}
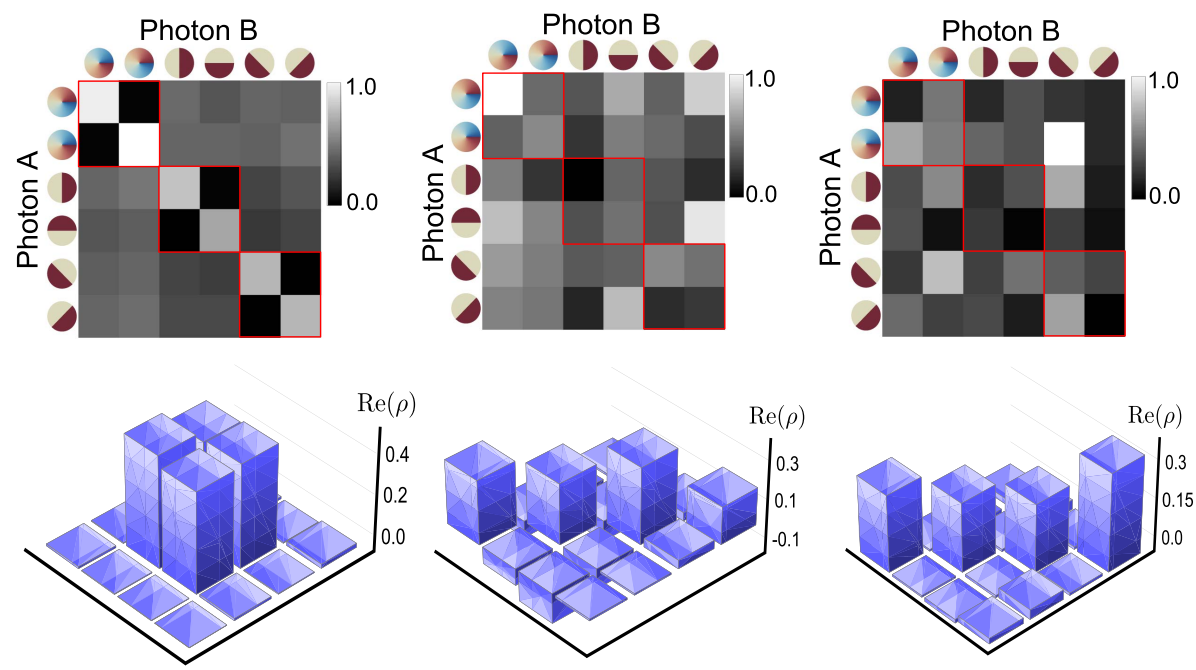

Examples of QST measurements and the resulting density matrices for hybrid (left) and OAM (middle and right) biphoton states, with varying degrees of entanglement.

\section{Figure 18}

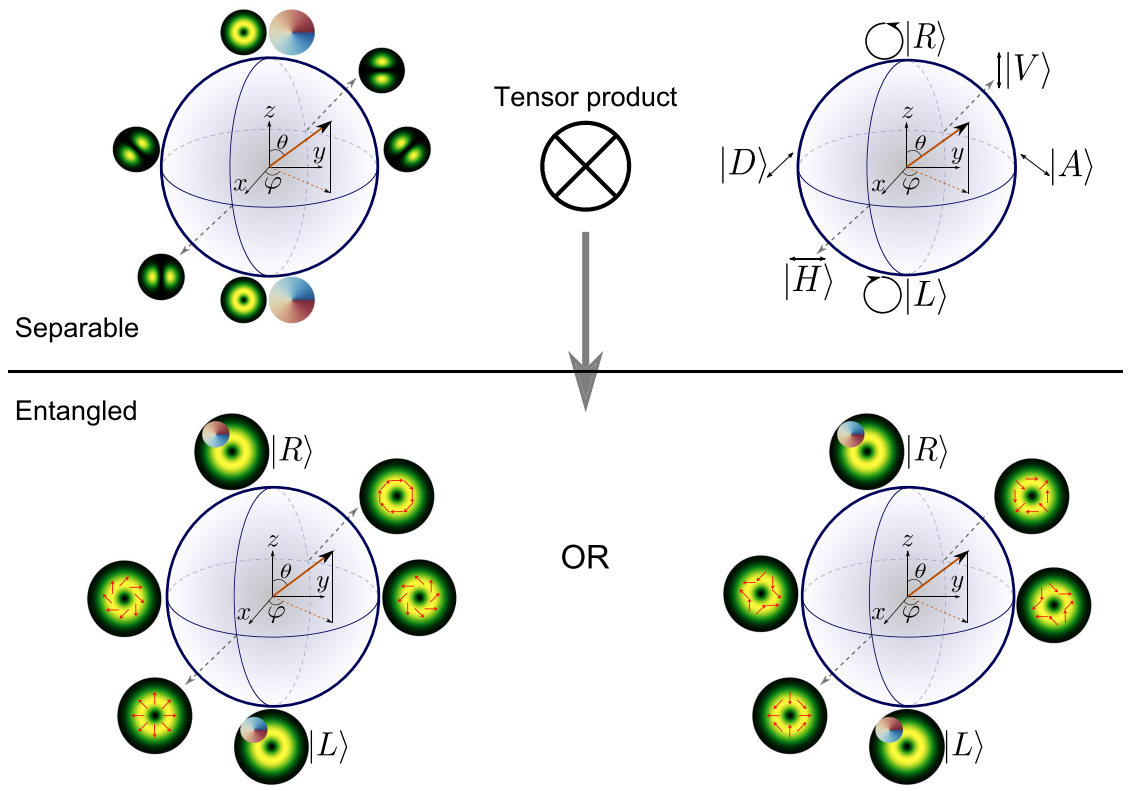

Description of hybrid states on the HOPS. States on the surface of the HOPS are constructed from the tensor product of OAM states from the Bloch sphere and polarization states from the Poincare sphere. The entire space is four dimensional but illustrated here by spheres representing the subspaces. The equator represents maximally entangled states, whereas the poles represent non-entangled states. 
where $\sigma_{m}^{\text {orbit }}$ and $\sigma_{m}^{\text {spin }}$ refer to the Pauli and identity matrices, whose eigenstates correspond to OAM and polarization states, respectively. To see how to perform the QST we return to Fig. 15; we see that we should select projections from each degree of freedom: spatial mode projections on photon A and polarization measurements on photon $\mathrm{B}$, to return the required data in the yellow block, following the same procedures outlined for biphoton qubits in a single degree of freedom. An example of such a measurement is shown in Fig. 19 for both maximally entangled and non-entangled hybrid states.

It is worth noting that the principles of QSTs presented here can be extended to higher dimensions. Beyond the qubit, one requires a generalization of the Pauli matrices to higher dimensions: these are the Gell-Mann matrices. However, the physical interpretations are not as straightforward as with the Pauli matrices and require more acrobatics. A recipe to construct these matrices is presented in Ref. [30] and has been used to perform state tomography of higher dimensional states [19]. In this tutorial we wish to demonstrate how to perform a QST with classical light, and, as we shall see, it is convenient to do so directly with two degrees of freedom, restricting us to qubit states.

\subsection{Extracting Information from QST Measurements}

There are many ways to characterize the purity and quality of quantum states once the density matrix has been reconstructed from a QST. Here we introduce some of the widely known measures, namely, the fidelity, linear entropy, and concurrence [149]. Importantly, one can analogously apply these measures to classically entangled states to test for non-separability between internal degrees of freedom of photons $[112,152,153]$.

First we introduce the fidelity that quantifies the equivalence or similarity between the reconstructed and target density matrices. It can be expressed as [154]

$$
F=\operatorname{Tr}\left(\sqrt{\sqrt{\rho_{1}} \rho_{2} \sqrt{\rho_{1}}}\right)^{2}
$$

where $\rho_{1}$ is the density matrix representing a target state and $\rho_{2}$ is the predicted (or reconstructed) density matrix. If the matrices are identical, then $F=1$; conversely, if they have no similarity, then $F=0$. Also note that this definition is generalized for both pure and mixed density matrices. For a target state that is pure, say $\rho_{1}=\left|\psi_{1}\right\rangle\left\langle\psi_{1}\right|$, Eq. (42) can be easily expressed as

\section{Figure 19}

(a)

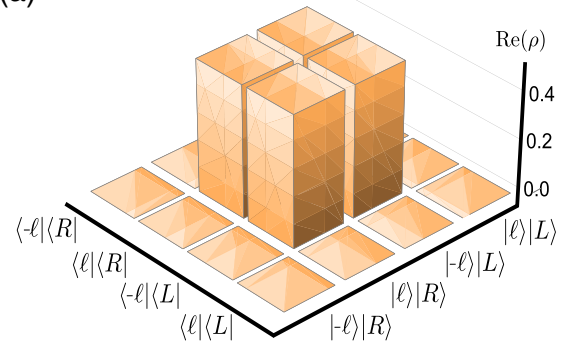

(b)

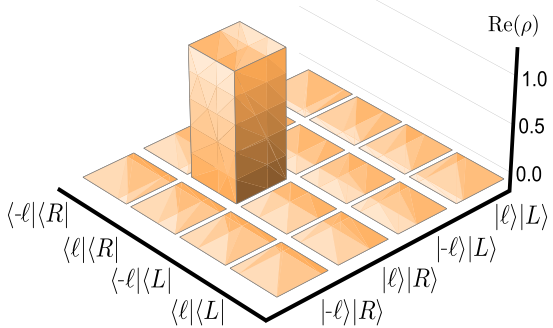

(a) Density matrix representation for a maximally entangled biphoton hybrid state and similarly in (b) for a hybrid state that is not entangled. 


$$
F=\operatorname{Tr}\left[\rho_{1} \rho_{2}\right]=\left\langle\psi_{1}\left|\rho_{2}\right| \psi_{1}\right\rangle
$$

reducing to a simple inner product between the two states. This then asks the question: is what I measured $\left(\rho_{2}\right)$ the same as $\rho_{1}$ ? Often the comparison is made to maximally entangled states so that the fidelity answers the question: is my state maximally entangled?

To illustrate how the fidelity is computed, suppose we have the density matrix shown in Fig. 19(a), which in matrix notation reads

$$
\rho_{1}=\frac{1}{2}\left(\begin{array}{llll}
0 & 0 & 0 & 0 \\
0 & 1 & 1 & 0 \\
0 & 1 & 1 & 0 \\
0 & 0 & 0 & 0
\end{array}\right)
$$

while Fig. 19(b) corresponds to the matrix

$$
\rho_{2}=\left(\begin{array}{llll}
0 & 0 & 0 & 0 \\
0 & 1 & 0 & 0 \\
0 & 0 & 0 & 0 \\
0 & 0 & 0 & 0
\end{array}\right)
$$

By applying Eq. (42) we obtain $F\left(\rho_{1}, \rho_{2}\right)=0.5$. Equivalently, the result can be interpreted as $\rho_{1}$ and $\rho_{2}$ having a 50\% overlap. We will use this measure as figure of merit for determining the overlap between density matrices.

Next we present the linear entropy. For any given density matrix, $\rho$, the linear entropy is expressed as

$$
\mathrm{S}_{L}=\left(1-\operatorname{Tr}\left(\rho^{2}\right)\right)
$$

We see that $S_{L}=0$ for the density matrices $\rho_{1,2}$ in Eqs. (44) and (45) since they satisfy $\operatorname{Tr}\left(\rho^{2}\right)=\operatorname{Tr}(\rho)=1$. This is generally true for pure states (entangled or separable). However, mixed states are not idempotent, i.e., $\rho^{2} \neq \rho$ and $0<\operatorname{Tr}\left(\rho^{2}\right)<1$. For a completely mixed state, that is, a sum of equally weighted pure state density matrices, the purity attains a minimum bound of $1 / d$, where $d$ is the dimension of the density matrix. Consequently, the linear entropy can be as large as $(d-1) / d$ for a maximally mixed density matrix. Moreover, $S_{L} \rightarrow 1$ as $d \rightarrow \infty$ for maximally mixed states.

Next, we present a measure for the degree of entanglement, namely, the concurrence, $C$. It can be expressed as [155]

$$
\mathrm{C}(\rho)=\max \left\{0, \sqrt{\lambda_{1}}-\sum_{i=2} \sqrt{\lambda_{i}}\right\},
$$

where $\rho$ is the density matrix of the system being studied (mixed or pure); $\lambda_{i}$ are the eigenvalues of the operator $R=\sqrt{\rho} \sqrt{\tilde{\rho}}$ in descending order, with $\tilde{\rho}=\Theta \rho^{*} \Theta$ where $*$ denotes complex conjugation. The operator $\Theta$ represents an anti-unitary operator satisfying $\langle\psi|\Theta| \phi\rangle=\left\langle\phi\left|\Theta^{-1}\right| \psi\right\rangle$ for any states $|\phi\rangle$ and $|\psi\rangle$ [149]. The concurrence can take values from 0 to 1 ; with no entanglement corresponding to a value of 0 , while an increasing degree of entanglement reaches values up to 1 .

As an example, we compute the concurrence of the density matrices from Eqs. (44) and (45) We use the following anti-unitary operator for a two-qubit state given by 


$$
\Theta=\sigma_{2} \otimes \sigma_{2}=\left(\begin{array}{cccc}
0 & 0 & 0 & -1 \\
0 & 0 & 1 & 0 \\
0 & 1 & 0 & 0 \\
-1 & 0 & 0 & 0
\end{array}\right)
$$

where $\sigma_{2}$ is the spin-flip Pauli matrix with $\Theta$ satisfying $\Theta^{-1}=\Theta^{\dagger}$. Accordingly, we compute $\tilde{\rho}$

$$
\tilde{\rho}_{1}=\frac{1}{2}\left(\begin{array}{cccc}
0 & 0 & 0 & 0 \\
0 & 1 & 1 & 0 \\
0 & 1 & 1 & 0 \\
0 & 0 & 0 & 0
\end{array}\right), \quad \tilde{\rho}_{2}=\left(\begin{array}{cccc}
0 & 0 & 0 & 0 \\
0 & 0 & 0 & 0 \\
0 & 0 & 1 & 0 \\
0 & 0 & 0 & 0
\end{array}\right)
$$

for matrices $\rho_{1,2}$ in Eqs. (44) and (45) [see Figs. 19(a) and 19(b) for illustrations], respectively. Note that $\tilde{\rho}_{1}=\rho_{1}$ but $\tilde{\rho}_{2} \neq \rho_{2}$. The $R$ matrix can thus be computed as

$$
R_{1}=\frac{1}{2}\left(\begin{array}{cccc}
0 & 0 & 0 & 0 \\
0 & 1 & 1 & 0 \\
0 & 1 & 1 & 0 \\
0 & 0 & 0 & 0
\end{array}\right), \quad R_{2}=\left(\begin{array}{cccc}
0 & 0 & 0 & 0 \\
0 & 0 & 0 & 0 \\
0 & 0 & 0 & 0 \\
0 & 0 & 0 & 0
\end{array}\right),
$$

each with eigenvalues $\{1,0,0,0\}$ and $\{0,0,0,0\}$ respectively. Subsequently the concurrence is computed from Eq. (47) for both states, yielding $C\left(\rho_{1}\right)=1$ and $C\left(\rho_{2}\right)=0$. Therefore, the density matrix $\rho_{1}$ corresponds is a maximally entangled state while $\rho_{2}$ corresponds to a separable state, as expected. Thus the concurrence suffices as figure of merit for the measure of the amount of entanglement in a quantum system. Furthermore, the concurrence can be simplified to $C(\rho)=\sqrt{2\left(1-\operatorname{Tr}\left(\rho_{B}^{2}\right)\right)}$, where $\rho_{B}$ is the reduced state in the case of pure states.

\section{SIMULATING QST MEASUREMENTS WITH SCALAR LIGHT}

The aforementioned examples of a QST were all performed on suitably entangled quantum states from a SPDC source. To understand how entanglement arises in the SPDC process, Klyshko put forward the idea of "time reversal" [122]. To see the implication of this rather abstract notion, consider the illustrations in Fig. 20. In the top panel we have the traditional quantum experiment: a noncollinear, degenerate SPDC process produces two photons (our biphotons), one in arm A and one in arm B. Each of the two entangled photons travels in a particular direction, here arms A and $\mathrm{B}$, until they are measured by some projection on the spatial light modulator (SLM), collected at the single-mode fiber (SMF), and resulting in a particular coincidence rate with the outcome of a similar process in arm B. Because of the phasematching condition of our SPDC crystal, the ejection angles of the biphotons at the crystal are equal and opposite, forming a ring-like structure of SPDC light. For our entanglement studies, we collect photons from diametrically opposite sides of the ring with suitably sized and placed apertures. Klyshko argued that to understand this process one could imagine one of the photons, say that in arm A, traveling backward in time, interacting with the crystal, and resulting in a new photon traveling in arm B. In this scheme the crystal is treated as a mirror. This works because mirrors reflect light at an angle equal to the angle of incidence, mimicking the SPDC phasematching condition for momentum. To realize this concept in the laboratory, one merely needs to replace the detector in one arm, say arm A, with a source of photons-a laser at the same wavelength as the biphotons. The bright laser beam will pass backward through arm A, reflect off the crystal surface (if flat and normal, otherwise a pop-up mirror), and then continue in arm B as if it were photon B through to the 
detector. Rather than two measurements on spatially separated photons (A and B), here we prepare the intense light in arm A to be in some state, and then detect the state in arm B.

This tool is often exploited for alignment of quantum experiments, but recently has been demonstrated as a versatile tool for actually predicting the outcome of quantum experiments, including full QSTs. It has been used to understand QSTs using spatial modes [118,156], to mimic pumping shaping of SPDC sources [121,157], to study losses in high-dimensional quantum key distribution systems [74], and to

\section{Figure 20}
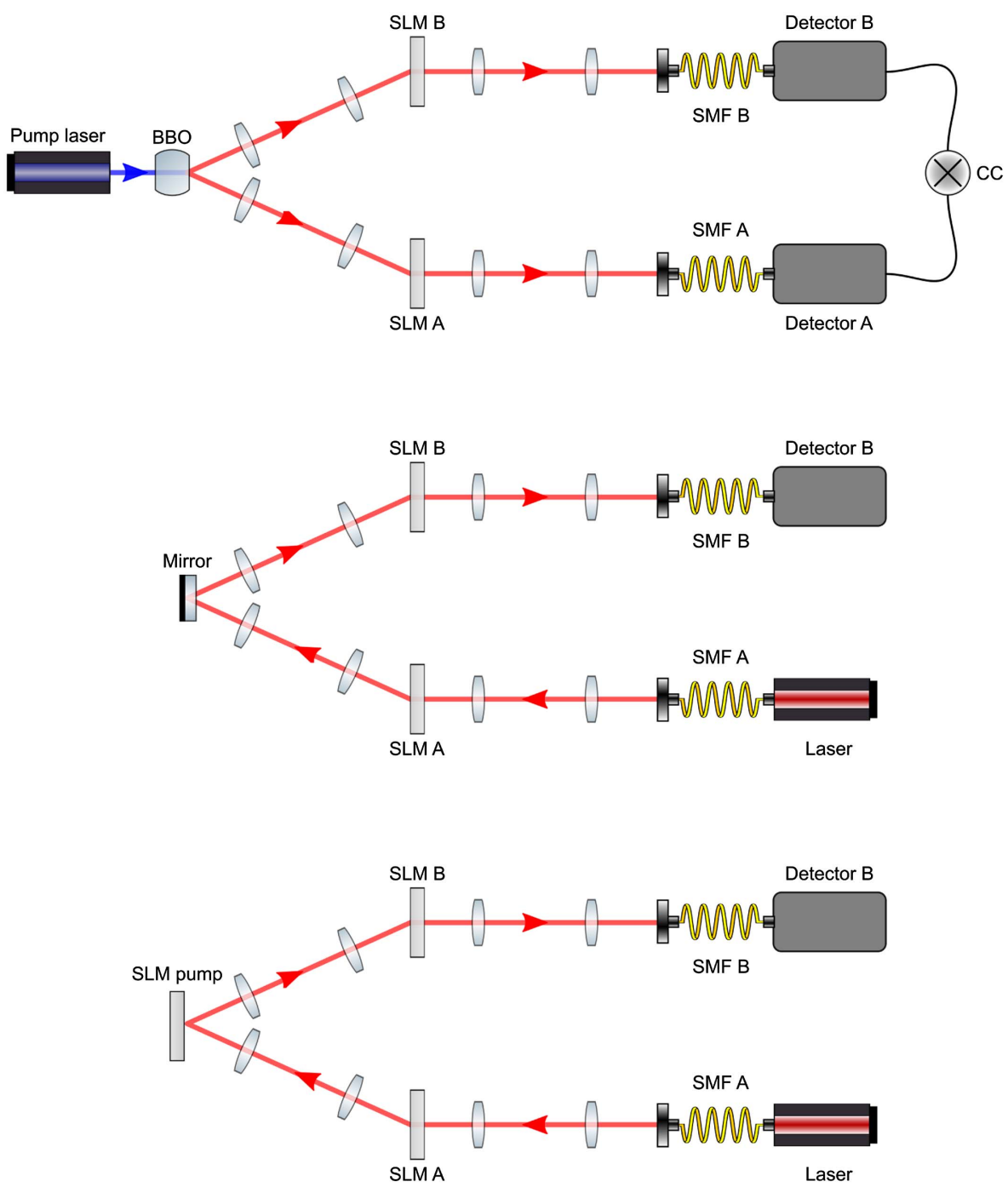

Top: conventional quantum experiment with biphotons using an SPDC source and projections using SLMs to explore spatial mode entanglement. Here two photons are produced at the crystal and travel in equal but opposite directions due to the crystal phase-matching condition. Middle: the detector in arm A is replaced with a source of bright light. The light travels backward through the system, bouncing off the crystal and passing through arm B to the detector. Because the angle of incidence equals the angle of reflection, the light in arm B has the correct properties to mimic the SPDC photon in this arm. Bottom: this concept can be further extended to simulate different SPDC processes by replacing the mirror by a third SLM, i.e., to simulate the mode of the pump beam. 
understand ghost imaging [117,119,158-162]. This approach is sometimes called "back-projection" [118].

To see why the QST is accurately mimicked, consider the example of OAM shown schematically in Fig. 21. The biphotons are created from a SPDC process that is excited with an $\ell=0$ pump, so that $\ell_{A}+\ell_{B}=0$. Here, if $\ell_{A}=1$ is the measurement in $\operatorname{arm} \mathrm{A}$, then a coincidence occurs only if the hologram in $\operatorname{arm} \mathrm{B}$ is given by $\ell_{B}=-1$. Now consider the classical light equivalent. The laser beam from source A passes through the same hologram A but backward, so that after the hologram the OAM state of the light is given by $\ell_{A}=-1$. After reflection from the mirror the helicity inverts, so the light is now $\ell_{A}=1$ and traveling toward hologram $\mathrm{B}$. This light now encounters the detection in arm $\mathrm{B}$, programmed as $\ell_{B}=-1$. Modulating an incoming OAM of $\ell_{A}=1$ with a hologram of $\ell_{B}=-1$ results in zero OAM since the helicity is removed. Since the SMF couples in light of $\ell=0$ (Gaussian beams with no OAM), the result is detection of the light. If the hologram in arm A was changed to $\ell_{A}=10$, then at the SMF the helicity of the beam would be $\ell=9$, which would result in no "click." One can run this thought experiment for all projections required for a QST and find that in all cases the classical experiment implements the process as it would be seen in the quantum case. In Fig. 22(a) we show results for OAM correlations performed on quantum states and on bright classical light, respectively, with full QSTs for the hybrid state shown in Fig. 22(b). It is clear that this is a very powerful tool to study QSTs with all the advantages of intense light, e.g., strong signals for fast and accurate QSTs. Further examples of higher dimensions and ghost imaging are shown in Figs. 23 and 24.

\section{Figure 21}

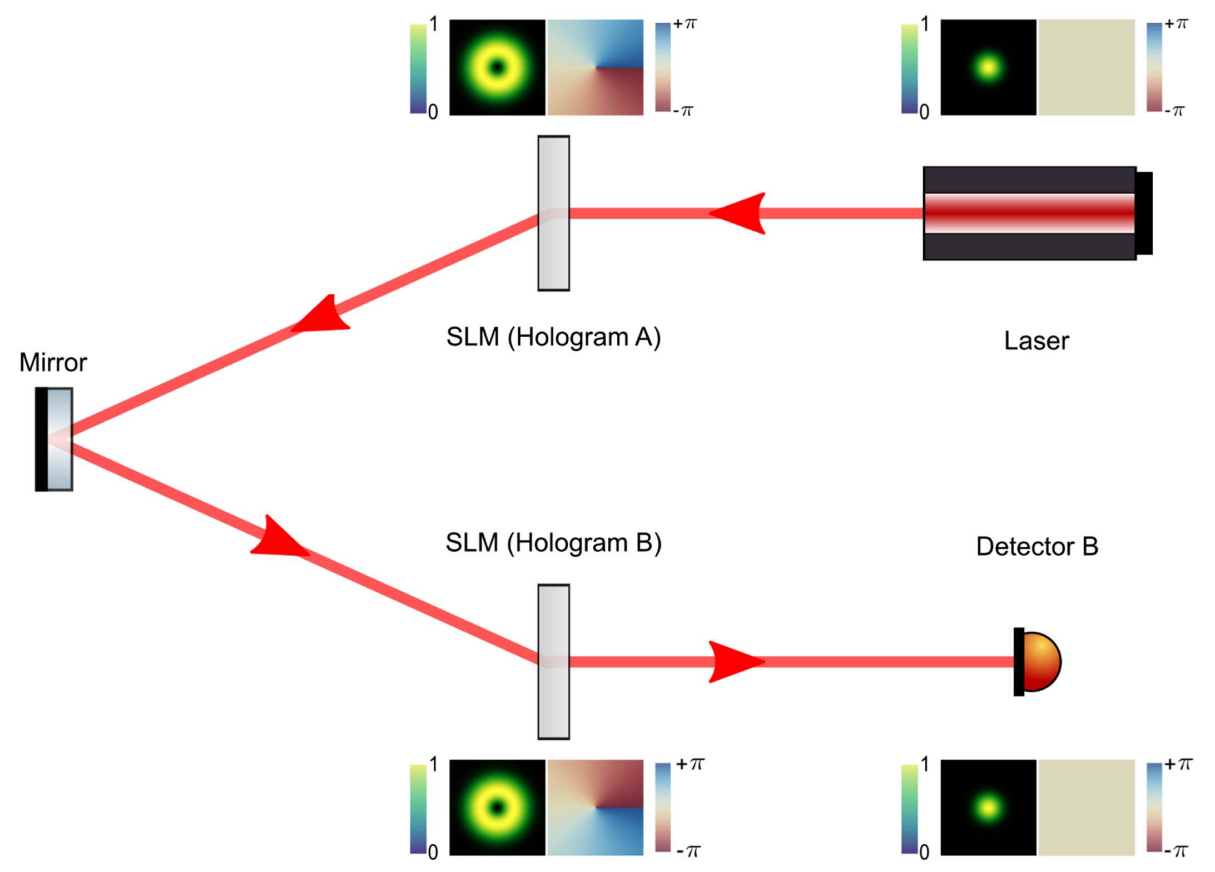

In the usual SPDC process the OAM modes in arms A and B have opposite sign due to conservation of OAM (assuming a Gaussian pump mode). In the classical experiment with one detector replaced by a laser, the extra reflection off the mirror suffices to flip the sign of the OAM that travels to arm B, thus mimicking the physics correctly for a QST. 


\section{QSTS WITH CLASSICALLY ENTANGLED LIGHT}

The hallmark of the biphoton quantum states that we have written in the earlier sections is their non-separability. This gives rise to the concept of entanglement: that a measurement on one photon affects the outcome of a measurement on the other. Now we will show that non-separability is not unique to quantum mechanics, and that this

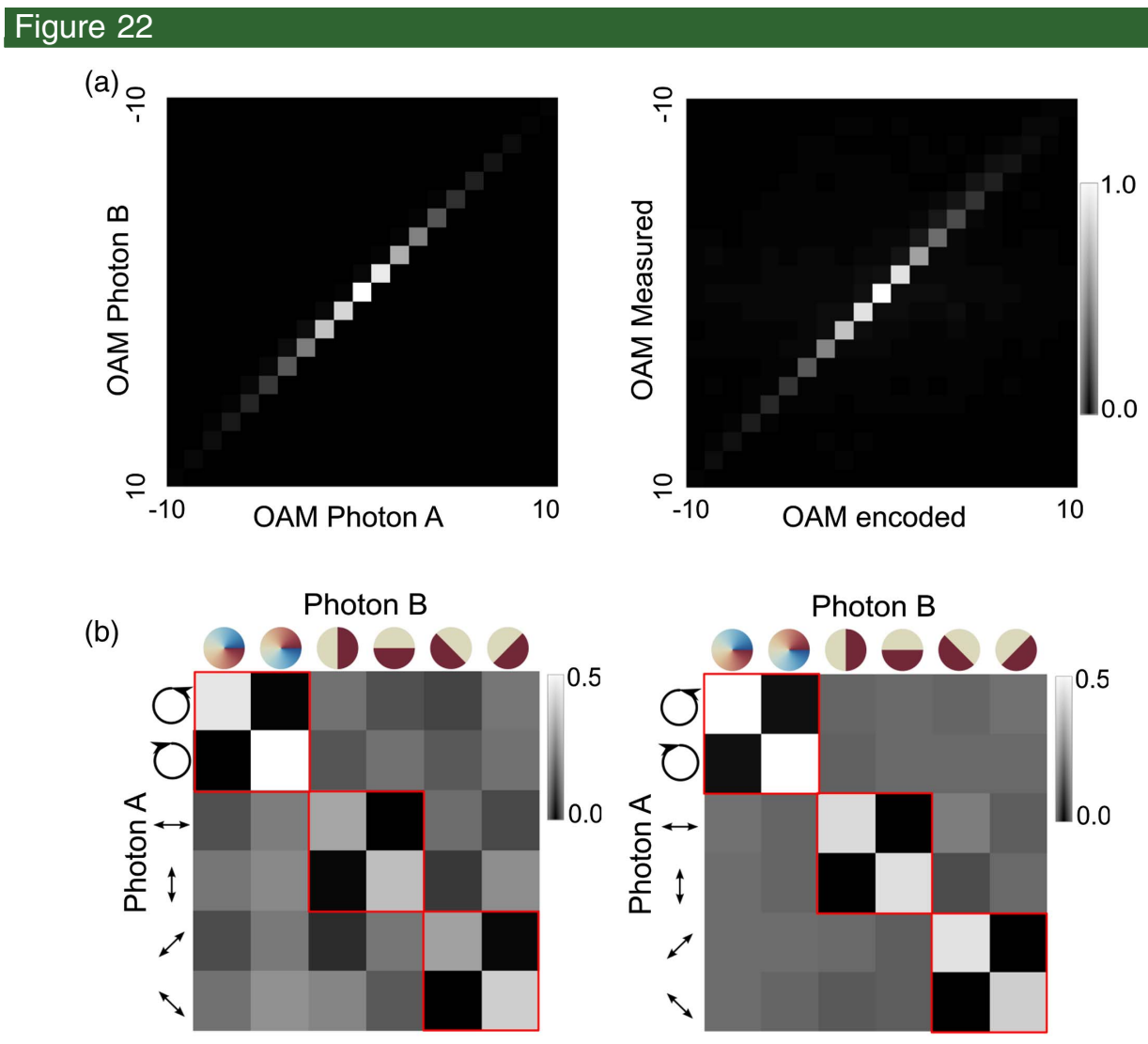

OAM spiral bandwidth shown for (a) the SPDC experiment with single photons (left) and the classical backprojection experiment (right). In (b) we show the outcome of a full QST (with differing degrees of freedom), again with quantum on the left and classical on the right.

\section{Figure 23}
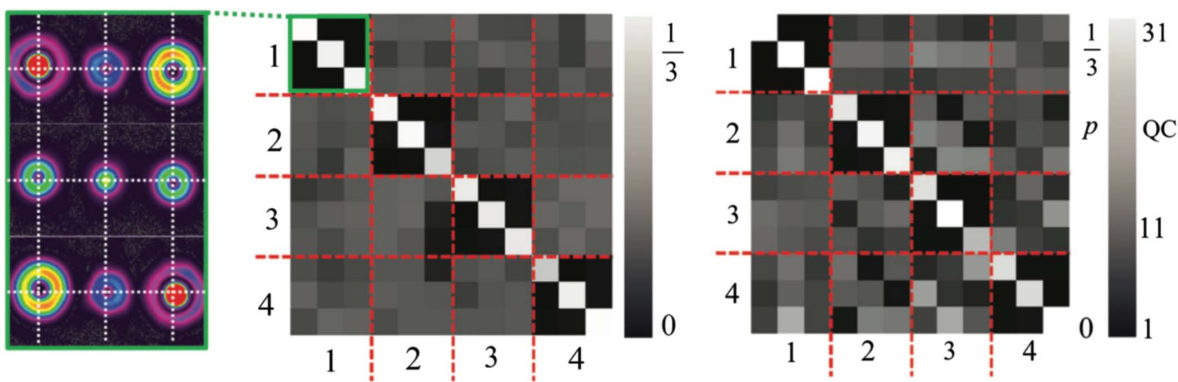

Example of actual classical signals at the detector (left) together with the resulting full QST on the classical beam (middle) and the corresponding quantum case (right). The agreement is excellent. Here the QST was performed on a $d=3$ Hilbert space. 
fact can be exploited to perform "quantum" measurements on purely classical light, observing all the salient features as if the state under study were really quantum. This facilitates a pedagogical approach to teaching QSTs with easy implementation for experimental demonstration.

Previously, we have used the density matrix approach to describe a quantum system of one and two qubits. However, this description is not in itself quantum; it is simply a mathematical tool to arrive at a physical description of reality. As such, it should also be applicable to non-quantum states. How does a single-qubit description vary from that of a classical state? The answer is not much, since photons are elementary excitation of the electromagnetic field. More interesting is the case of two entangled photons: can this be described classically?

Through "spooky action at a distance," the states of two systems can be coupled in a non-separable manner, such that they cannot be described independently from each other, regardless of how far apart they are located. One way to test the "quantumness" of correlations arising from entanglement is through the CHSH inequality, and this has successfully been demonstrated experimentally [163]. However, how does the nature of the entangled parties come into the description of entanglement? We have described entanglement correlation as existing between two distinct systems that can be physically separated. What is the physical reason behind such a requirement? Can nonquantum systems exhibit entanglement correlations? These questions have become topical recently $[105,106,108,109,164,165]$. In this section we will present our view

\section{Figure 24}

Position configuration
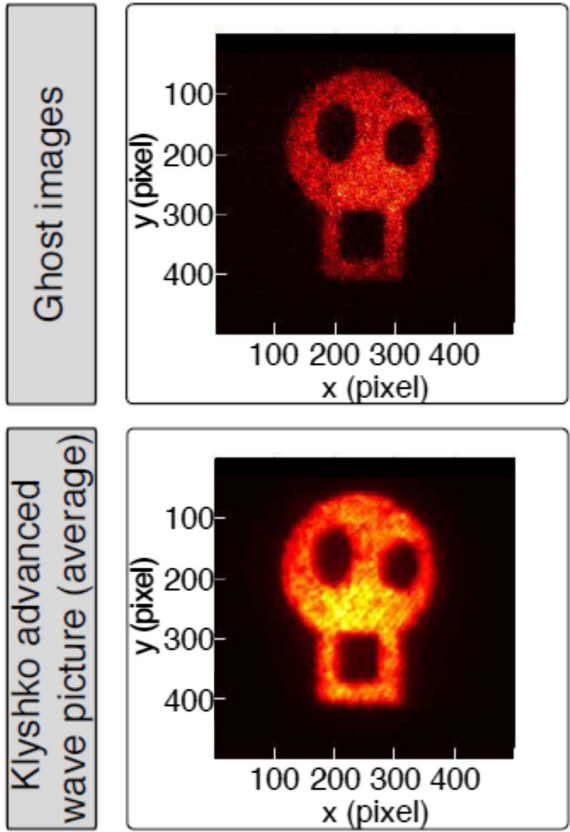

\section{Momentum configuration}
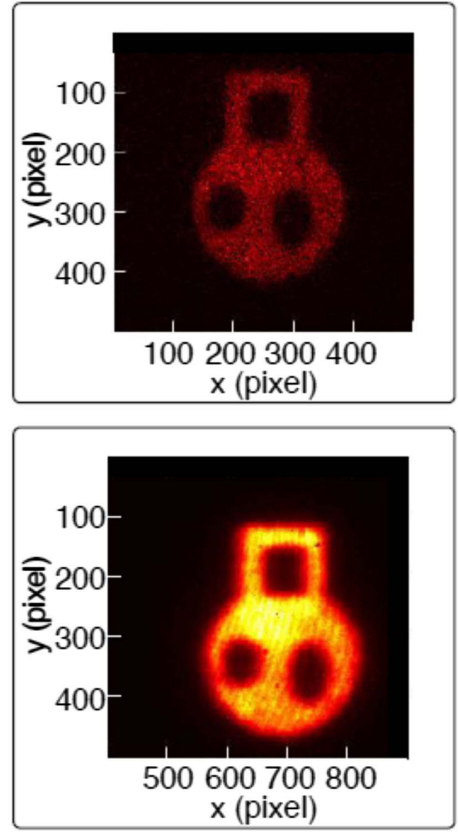

The backprojection approach can also be used to demonstrate other quantum experiments, for example, ghost imaging. Here we illustrated examples of ghost imaging with position and momentum correlations using SPDC photons as well as a classically equivalent backprojection experiment. Copyright 2014 from "Experimental demonstration of Klyshko's advanced-wave picture using a coincidence-count based, camera-enabled imaging system," Aspden et al. [119]. Reproduced by permission of Taylor and Francis Group, LLC, a division of Informapic. 
on the issue and, more practically, show how to exploit so-called classical entanglement for research and teaching purposes.

\subsection{What is Entanglement?}

The quintessential property of entanglement is non-separability, the notion that two systems cannot be described independently from each other, or put another way, that a measurement on one system is strongly (i.e., beyond classical correlations) linked to the outcome of the measurement of the other. Mathematically we say that the joint state does not factorize; that is, given two systems $A$ and $B$, the joint density matrix $\rho_{A B}$ cannot be expressed as the tensor product of the individual density matrices $\rho_{A}$ and $\rho_{B}$. Thus any state that is not separable is said to be entangled. Note at this point that we have not specified anything about the nature of the systems $A$ and $B$ (whether they are classical or quantum). Yet, we have logically arrived at a condition for separability and entanglement. So where does the "quantumness" of entanglement originate? The reason is none other than context. We believe the issue lies in understanding what is entangled.

Suppose you were to deliver pairs of identically prepared entangled photons to two experimentalists, Alice and Bob, who are both tasked to test for violation of the $\mathrm{CHSH}$ inequality. In our picture, Alice and Bob cannot agree on what to measure: Alice wishes to perform her measurement in the polarization basis, while Bob prefers to do it in the spatial degree of freedom basis. They both proceed and return their results to a third party, Eve, as one of two possibilities: "entangled" or "not entangled." Eve can draw the following conclusions:

(1) Both or none of the outcomes handed by Alice and Bob show the violation of the $\mathrm{CHSH}$ inequality. In this case, both experimentalists agree and Eve can logically confirm or rebuke the presence of entanglement.

(2) Either one of the outcomes handed by Alice or Bob show the violation of the CHSH inequality. In this case, Eve is in a difficult position: by not knowing whether Alice and Bob performed an entanglement test using the same basis (i.e., whether their measurement was made using different degrees of freedom), she is faced with ambiguous and potentially conflicting results, and thus cannot establish the presence (or absence) of entanglement.

Therefore, it is imperative that one specifies the degree of freedom in which the photons are entangled. This brings us back to the question of what it is that is entangled: are the photons entangled? Or are the states of the photons entangled? Based on our imagined experiment involving Bob, Alice and Eve, it is clear that it is the latter: the states are entangled and the objects that happen to carry those states are not! This is an important distinction because it brings us to a current topical issue, that of classical entanglement: can a classical state of light be entangled? We can now reformulate the question in terms of our criterion for separability, namely, "Is it possible to construct a classical state of light that is non-separable?" If the answer to that question is yes, then the classical state can be said to be entangled in the non-separable degree(s) of freedom, which in the case of vector modes would be polarization and OAM, as discussed in the next section.

\subsection{Non-Separability, Vector Beams, and Classical Entanglement}

Optical fields may be scalar or vector, with the latter now topical classical states of light $[166,167]$. For a thorough and up-to-date discussion of vector beams, the reader is directed to the review article by Rosales-Guzmán et al. [168], and all references therein. The vector nature of these beams stems from the fact that they possess 
spatially varying polarization in the transverse plane, i.e., inhomogeneous polarization states of light. This coupling of space to polarization can be expressed as a non-separable superposition of spatial mode and polarization. To see what this means, consider the example vector beams shown in Fig. 25(b). If one of them, say the first on the left (radially polarized light), were passed through a polarizer, the intensity pattern observed by a detector (for example a camera) would change, shown in Fig. 25(c). We understand this as filtering out only particular polarization directions from the field. Put into the language of quantum mechanics: a measurement on one system affects the outcome of a measurement on the other. In other words, the measurement on the spatial mode (as observed by the camera) was affected by the prior choice of a measurement on the polarization (as performed by the polarizer). In contrast, this description does not apply to scalar beams, which are completely separable (i.e., the spatial properties are not affected by a change in polarization). For instance, inserting a polarizer in the path of a scalar beam will cause the intensity detected by the camera to globally increase or decrease according to the angle of the polarizer, but the spatial pattern of light as detected by the camera will not be affected. We therefore say that the spatial and polarization states of scalar modes are completely separable, as one does not affect the other.

\section{Figure 25}

(a)

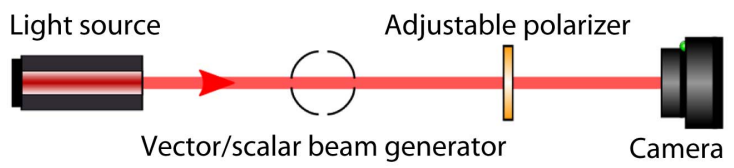

(b)

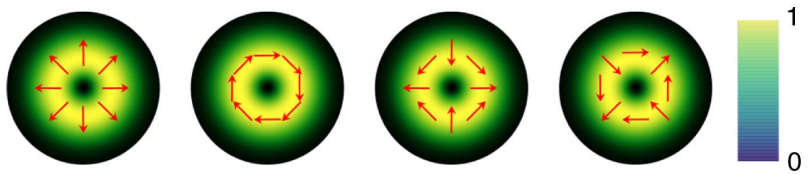

(c)

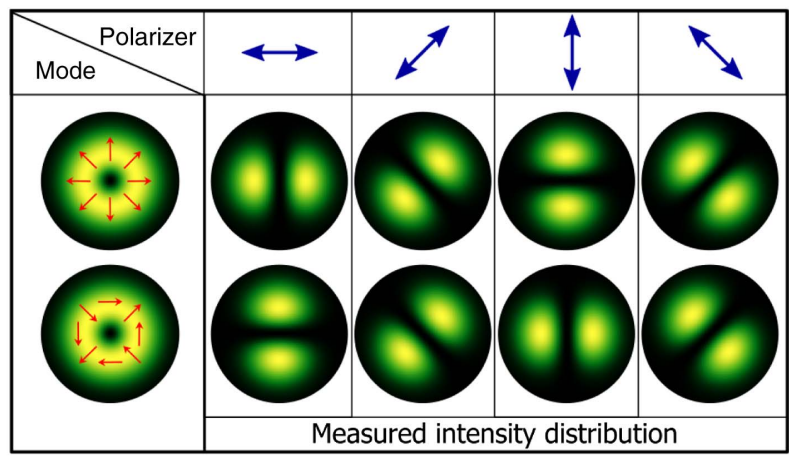

(a) Illustrative experiment able to reveal the separability and non-separability of vector and scalar modes, consisting of a light source, a vector/scalar beam generator, an adjustable polarizer, and a spatially resolved camera detector. (b) Intensity and polarization map of a few vector beams. The non-separability of the space and polarization degrees of freedom is reflected in the space variant (inhomogeneous) polarization. (c) The non-separability of the two degrees of freedom manifests itself if one passes the beam through an adjustable polarizer and measures the resulting spatial mode on a camera as the polarizer is rotated, as represented here for two vector beams. 
The vector beams shown in Fig. 25(b) can be conveniently expressed using a notation borrowed from quantum mechanics as follows:

$$
|\psi\rangle=\cos (\theta / 2)|\ell\rangle_{A}|R\rangle_{B}+\exp (i \varphi) \sin (\theta / 2)|-\ell\rangle_{A}|L\rangle_{B}
$$

Note that this expression of a vector beam is reminiscent of that in Eq. (36), which we used to describe an arbitrary state on the surface of the higher order Bloch sphere. One of the conclusions we drew was that such a state is non-separable, and we can now call it an entangled state. In Eq. (40) we saw that the non-separability of a hybrid entangled state was expressed across two photons, A and B. Here we have only one intense beam of light that is non-separable in two degrees of freedom: A and B. Once again we highlight that entanglement is the presence of a strong correlation between states. Thus we can conclude that a vector beam also carries a form of entanglement in its two degrees of freedom, albeit local. We call this "classical entanglement."

Entanglement between the quantum states of two particles exhibits non-locality: it persists even when the particles are space-like separated; that is, the particles are sufficiently far apart that in the event of a joint measurement, a field would need to propagate faster than light from one detector to the other to influence the measurement outcome. It is because of this non-locality that entanglement cannot be classified as exchange of information between the correlated particles; measuring the OAM of one photon in the OAM entangled pair does not result in a signal traveling to the other photon that tells it to assume a particular state. Such non-locality does not exist in the classically entangled fields we are describing here.

The degrees of freedom of the vector beam are defined locally and thus cannot exhibit non-local correlations. So can one still maintain that vector beams are entangled states? Yes, because the definition of entanglement was linked to the separability of the state and made no mention of non-locality. The issue of non-locality arose only when we added additional constraints to the description of the system: we specified the nature of the carriers of the entangled state. It is only when the entangled states are given properties of particles that the issue of non-locality emerges. When considering an inherently local system there is naturally no chance of non-local correlations. This being said, correlations do still exist in both systems. It is thus useful to make a distinction between these two "flavors" of entanglement: i.e., quantum and classical entanglement. More specifically, since non-local correlations are inherently quantum, we can logically refer to the entanglement of quantum particles as quantum entanglement and that of local classical systems, such as vector beams, as classical entanglement.

\subsection{Controlled Classical Entanglement by Spin-Orbit Coupling}

How should we produce such controlled "classically entangled" vector beams? The task is to do so in a manner that is tunable, moving freely around the HOPS, from separable modes on the poles to non-separable modes on the equator. Further, we wish to do so with intense light fields. It is possible to do so by employing the tools of structured light in general [134], for example, with dynamic phase using SLMs [169], directly from custom lasers [170], and by the use of spin-orbit coupling [171-173]. Devices called spin-to-orbital angular momentum converters (SOCs) allow to convert a Gaussian beam into a helical beam with an OAM related to the spin state of the input light, as illustrated in Fig. 26(a). This is possible in optically inhomogeneous and anisotropic media, such as oriented liquid crystals [91,174,175-178].

A completely different approach consists in using metasurfaces [179-181]. The general idea of metasurfaces is to have a nanostructured interface to shape the light 
wavefront at will [182,183], being able to implement not only SOCs but also lenses, axicons, and even ultrathin gratings $[184,185]$. For mid-IR light, metallic nanostructures can be used [186]. However, metals are too lossy in the visible, where they must be replaced by dielectric materials. A possible design approach for a dielectric metasurface in the visible is based on nanoposts made of a dielectric material [187] that has high refractive index and low losses in the visible. Later in this tutorial we will demonstrate how to perform a QST with intense laser light in the visible. With this in mind, our material of choice is titanium dioxide $\left(\mathrm{TiO}_{2}\right)$ with a refractive index of $\sim 2.4$ at green wavelengths $(\lambda=532 \mathrm{~nm})$ and negligible losses in the whole visible range [180]. When the dimensions of such dielectric nanoposts are smaller than the light wavelength, each post behaves like a truncated waveguide with most of the light just passing through, with negligible reflection at the interfaces. For a fixed height of the

\section{Figure 26}

(a)
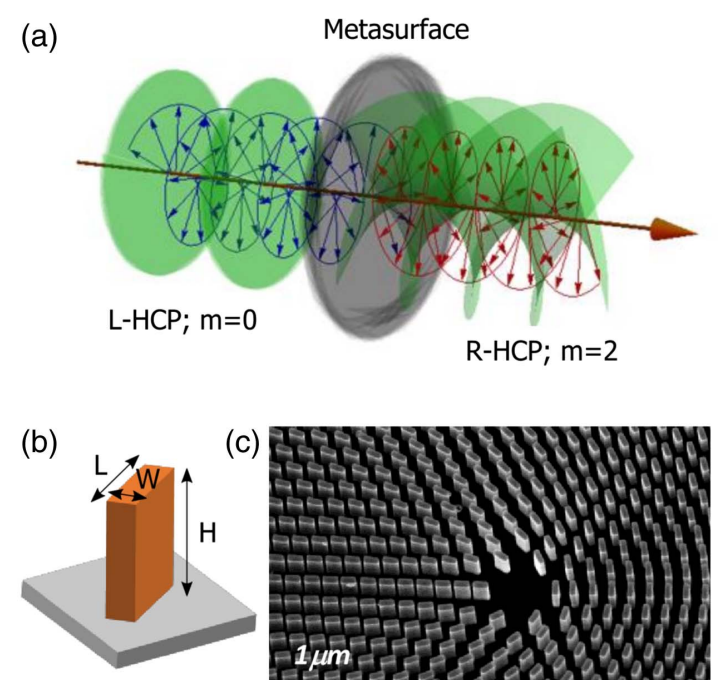

(c)
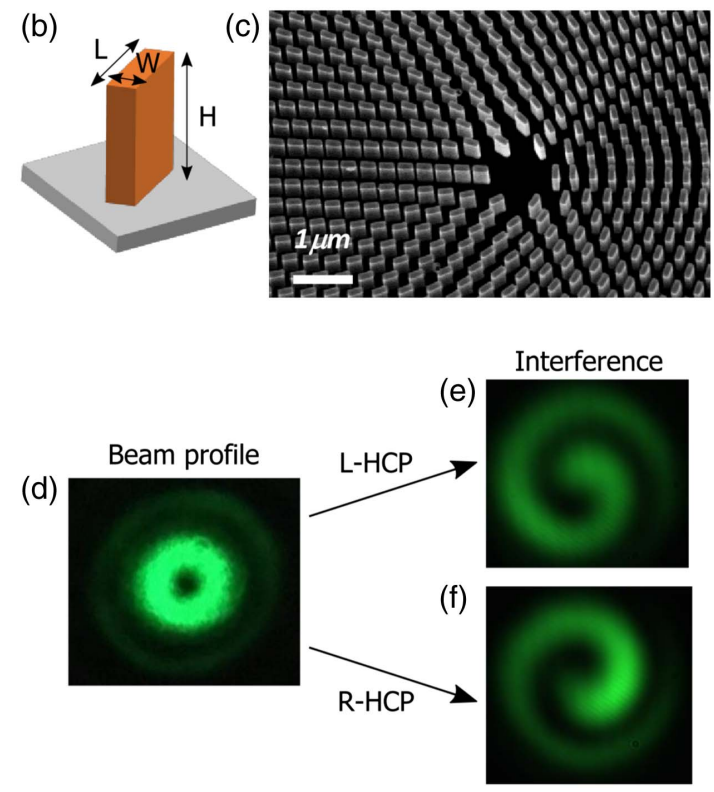

(a) Representation of a SOC based on a dielectric metasurface for visible light. This device converts a left-circularly polarized light Gaussian beam into a right-circularly polarized helical beam with OAM $m=2$. (b) Design of a rectangular-section dielectric nanopost (nanofin). If the material is $\mathrm{TiO}_{2}$, and the height of the post $(H)$ is $600 \mathrm{~nm}$, in order to achieve structural birefringence value of $\pi$ at $532 \mathrm{~nm}$ incident wavelength, the width (W) and the length (L) must be, respectively, 90 and $250 \mathrm{~nm}$. (c) SEM micrograph of a $\mathrm{TiO}_{2}$ metasurface $\mathrm{SOC}$ that produces helical beams of OAM \pm 1 . (d) Beam profile generated by the device in (c). (e) and (f) Interference of the helical beam in (d) with a reference beam. The spiral interference handedness depends on the sign of the helical beam OAM. 
post, the width and length can be then adjusted to impose a certain phase delay during the propagation. Let us call this propagation phase $(\Psi)$. In the case that the transverse section of the nanopost is not cylindrically symmetric (width and length are different), as shown in Fig. 26(b), also a phase delay $(\Delta \Phi)$ can be accumulated between the field components along the axes of the post. If this is the case, the nanopost behaves like a waveplate with an effective birefringence $(\Delta \Phi)$, called form or structural birefringence. The effect of a nanopost oriented by the angle $\alpha$ to the propagating light can be described by the Jones formalism as

$$
R(-\alpha)\left(\begin{array}{cc}
e^{i \psi} & 0 \\
0 & e^{i(\psi+\Delta \Phi)}
\end{array}\right) R(\alpha)
$$

If circularly polarized light, left or right, passes through each of such elements and the structural birefringence is fixed at a value $\Delta \Phi=\pi$, the output fields become

$$
\begin{gathered}
\mathbf{E}_{\text {in }}=\left[\begin{array}{l}
1 \\
i
\end{array}\right] \rightarrow \mathbf{E}_{\text {out }}=e^{i \psi} e^{-i 2 \alpha}\left[\begin{array}{c}
1 \\
-\mathrm{i}
\end{array}\right], \\
\mathbf{E}_{\text {in }}=\left[\begin{array}{c}
1 \\
-\mathrm{i}
\end{array}\right] \rightarrow \mathbf{E}_{\text {out }}=e^{i \psi} e^{i 2 \alpha}\left[\begin{array}{l}
1 \\
i
\end{array}\right] .
\end{gathered}
$$

The output light is then still circularly polarized but with opposite handedness with respect to the input field. Moreover, the output beam has accumulated an overall phase that depends not only by the propagation phase $(\Psi)$ but also by the orientation of the element $( \pm 2 \alpha)$. If we now place on a surface all equal nanoposts but differently oriented [Fig. 26(c)], the wavelets emerging from each nanopost are dephased only by means of the orientations $2 \alpha$. This phase term is a particular manifestation of the Pancharatman-Berry (PB) phase or geometric phase (see Ref. [188] for a popular review). Note that here, $\alpha \equiv \alpha(x, y)$, i.e., it is a spatially variant function according to the desired phase change. For example, to generate a helical beam with OAM $|m|$, the azimuthal phase gradient $m \phi$ must be imposed:

$$
2 \alpha(\phi)=m \phi .
$$

This results in a metasurface made of identical nanoposts, each with an orientation given by the equation above. Figure 26(c) shows a scanning electron microscope (SEM) image of a device that converts right- and left-circularly polarized light into left- and right-circularly polarized light and angular momentum +1 and -1 , respectively. Such a metasurface-based device has several advantages with respect to the equivalent liquid crystal devices in terms of reproducibility, degradation, and accuracy in encoding the azimuthal phase profile as well as the complexity of the possible designs. These devices are fabricated by means of electron beam lithography followed by atomic layer deposition (see Ref. [180] for further details on the fabrication process)

The metasurface approach also allows more exotic conversions with a single device. In fact, the propagation phase can also be made a of the azimuthal coordinate $\Psi \equiv \Psi(\phi)$. With the metasurface design there is then an extra "knob" to adjust to make more complex transformations. For example, a device can be produced to convert two circularly polarized Gaussian beams into helical beams of opposite spin momentum and arbitrary values of OAM. For instance, Fig. 27(a) shows the SEM micrograph of part of a device that converts left-circularly polarized light into a right-circularly polarized helical beam with OAM 3, while it converts right-circularly polarized light into 
a left-circularly polarized helical beam with OAM 6. In this case, since both the PB phase and the propagation phase have to change in the transverse plane, the nanoposts are not just differently oriented, but they also have different widths and lengths. Such device allows then transforming the spin base into an arbitrary subspace of the total angular momentum $J$ [93]. For this reason, these recently demonstrated devices are called $J$-plates. An example of a transformation allowed by a $J$-plate designed for circularly polarized input states is generated when such device is illuminated with linearly polarized light. In this case in the spin base, the input beam polarization state

\section{Figure 27}
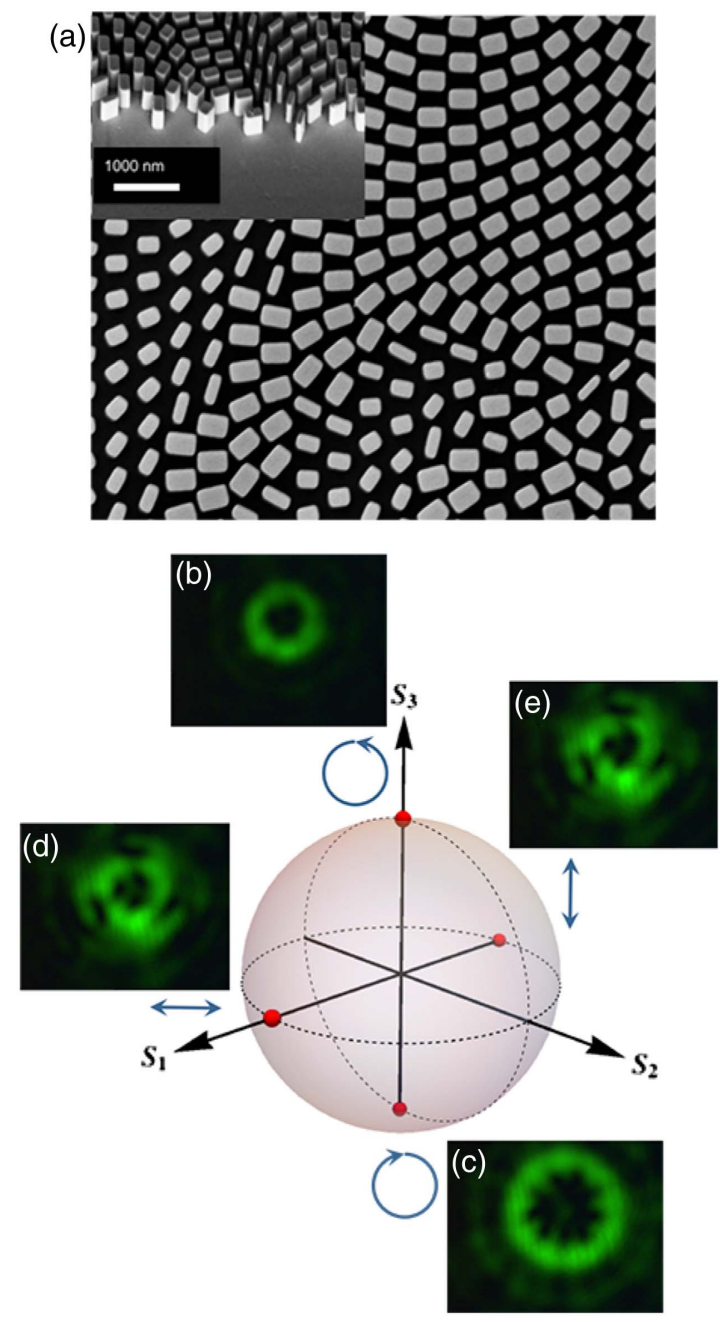

(a) SEM image of a $J$-plate. This dielectric metasurface device is made of different and differently oriented nanoposts with the same height. This allows to control both the propagation phase and the geometrical phase to map the spin base into an arbitrary subspace of the OAM. For instance, as represented on the HOPS, left-circularly polarized light is converted into right-circularly polarized light with $\mathrm{OAM}+3$, while right-circularly polarized light is converted into left-circularly polarized beam with OAM of +6 , shown in (b) and (c). When the input polarization state is not a pure state, both helical beams are generated. These two beams are in orthogonal polarization states and do not interfere. However, when they are both projected into the same polarization state by means of a polarizer, they produce complex interference pictures. (d) The interference picture obtained from incident horizontal polarization state. (e) The same as (d) when the incident light is vertically polarized. 
has complex weights that depend on the orientation of the polarization direction [Eq. (2)]. The output beam is then a complex superposition of two helical beams with different OAM, as shown in Figs. 27(d) and 27(e).

The design of a $J$-plate can be further generalized to accept any pair of orthogonal polarization states as input states, not necessarily the spin base. A $J$-plate can be designed to work, for instance, with input linear polarization states as well as with two orthogonal elliptical polarization states. However, to obtain such general mapping, making both the PB phase and the propagation phase function of the nano-element position on the surface is not enough. Such degree of conversion needs to also eventually change the birefringence of each nano-element [93]. Such condition in fact means that the wavelets from each of the nano-elements are potentially in different polarization states. Considering that each nanopost has subwavelength transverse dimensions, i.e., millions of them are illuminated by the incident beam simultaneously, a $J$-plate allows to generate complex polarization patterns in the output beam, i.e., complex vector beams.

The result of such devices is the ability to access arbitrary HOPS of a more general form. First, having shown the ability to create arbitrary intense beams with variable "entanglement," we need to complete the tool-kit with the classically equivalent detection of such beams.

\subsection{Exploiting the Mathematical Similarity}

Given the mathematical similarities between quantum entangled states and vector beams, there has been interest in using classical states of light to model local quantum entanglement dynamics $[104,189,190,191]$. This requires some commonality between the quantum and classical systems in the way they are analyzed to make a fair comparison. In the previous section we have identified a QST as the tool of choice for quantum state reconstruction. Fortunately, the procedure behind a QST of a biphoton state can be directly applied to vector beams [152,153,192,193] following the same approach as for the hybrid entangled states. This is because of the mathematical equivalence of the two functions. The only adaptation is in terms of the projective measurements required: now in series on one classical beam rather than on two photons. So let us go through the procedure of the tomographic measurement of the state of a vector beam. Recall the expression of a vector beam:

$$
|\psi\rangle=\cos (\theta / 2)|\ell\rangle|R\rangle+\exp (i \varphi) \sin (\theta / 2)|-\ell\rangle|L\rangle \text {. }
$$

We have shown previously that the above state can be described as a point on the surface of a sphere. We have demonstrated that the sphere is constructed from the tensor product of the eigenstates of the individual subsystems. Here, the two systems correspond to degrees of freedom of a classical beam; these are hybrid states of OAM and polarization. Previously we expressed the density matrix of a two-photon hybrid state as

$$
\rho=\left(\frac{1}{2} \sum_{m=0}^{3} \rho_{m} \sigma_{m}\right)^{\text {orbit }} \otimes\left(\frac{1}{2} \sum_{n=0}^{3} \rho_{n} \sigma_{n}\right)^{\text {spin }}=\frac{1}{4} \sum_{m, n=0}^{3} \rho_{m n} \sigma_{m}^{\text {orbit }} \otimes \sigma_{n}^{\text {spin }}
$$

and now we see that this must work for our vector beam, too, except that we have two degrees of freedom rather than two photons. Similar to the biphoton case, one can place the vector beam on the surface of one of two distinct spheres, known as HOPS $[123,125,194]$. The states on the poles are separable eigenstates of OAM and polarization and can be of the form 


$$
\begin{aligned}
& \left|S_{1}\right\rangle=|\ell\rangle|R\rangle, \\
& \left|S_{2}\right\rangle=|\ell\rangle|L\rangle, \\
& \left|S_{3}\right\rangle=|-\ell\rangle|R\rangle, \\
& \left|S_{4}\right\rangle=|-\ell\rangle|L\rangle,
\end{aligned}
$$

spanning a four-dimensional space. The states on the equator are of particular interest, as they correspond to maximally entangled vector beams, otherwise known as the cylindrical vector vortex beams, of which four common examples are the fiber modes:

$$
\begin{aligned}
|\mathrm{TM}\rangle & =\frac{1}{\sqrt{2}}(|\ell\rangle|R\rangle+|-\ell\rangle|L\rangle), \\
|\mathrm{TE}\rangle & =\frac{-i}{\sqrt{2}}(|\ell\rangle|R\rangle-|-\ell\rangle|L\rangle), \\
\left|\mathrm{HE}_{o}\right\rangle & =\frac{1}{\sqrt{2}}(|\ell\rangle|L\rangle+|-\ell\rangle|R\rangle), \\
\left|\mathrm{HE}_{e}\right\rangle & =\frac{i}{\sqrt{2}}(|\ell\rangle|L\rangle-|-\ell\rangle|R\rangle) .
\end{aligned}
$$

These spatial modes have been used in various applications, such as quantum metrology and communication [89,103,104,138,195-200].

The projective measurements necessary for a QST of the classical vector beam are the same as those of the biphoton case, with the exception that one set of eigenstates corresponds to polarization and the other to the spatial mode, as depicted in Figs. 28(a) and 28(b). This is the same approach as was explained earlier with

\section{Figure 28}

(a)
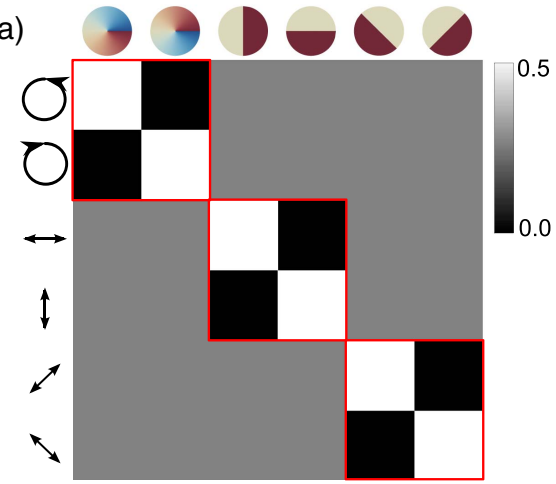

(b) 0000

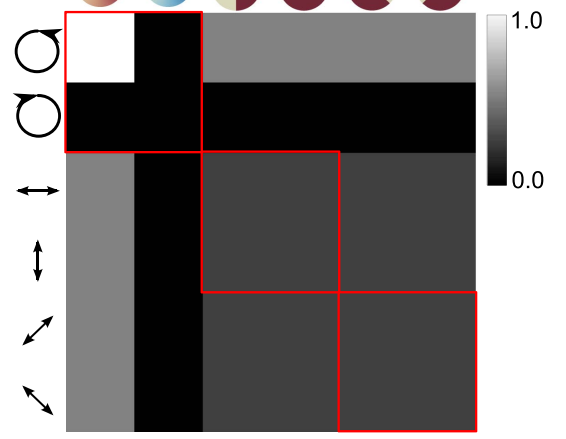

(c)

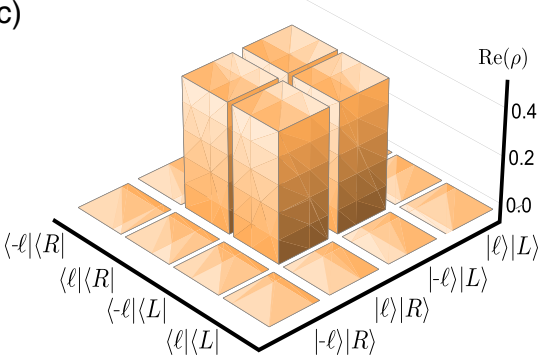

(d)

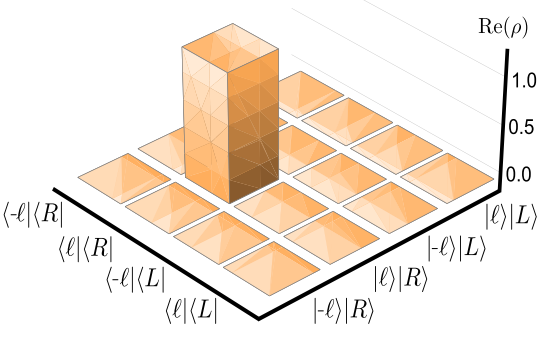

Graphical representation of the tomographic projections onto both eigenstates of the OAM and polarization Pauli matrices for (a) a maximally non-separable vector mode and (b) a left-circularly polarized OAM mode with $\ell=1$ (scalar mode). (c) and (d) show the reconstructed density matrices for the classical vector and scalar modes in (a) and (b), respectively. 
the aid of Fig. 15. Rather than two photons, A and B, we replace "photons" with "degrees of freedom." Then the measurement of the hybrid quantum state and the classically entangled vector beam are identical. Let us return to the usual entanglement case, say biphotons entangled in OAM: we have one degree of freedom and two photons. The QST is then based on projections in one degree of freedom on each of the two photons, following Fig. 15. In the classically entangled case we have two measurements on one intense beam of light. In the former, the measurements are in general non-local, while in the latter they are local. The measurements again follow the rules of Fig. 15.

Consider the outcome of this process, shown in Fig. 28(a). The set of measurements from the eigenvalues of the identity operator $\sigma_{0} \otimes \sigma_{0}$ are shown to be enclosed by one of the red squares; this is a consequence of the completeness of projectors that ensure conservation of probabilities. From these measurements, one computes the density matrix, whose real part is shown in Fig. 28(c).

In the context of QST, the joint measurements $\left\langle\lambda_{i}^{m} \lambda_{j}^{n}|\Omega| \lambda_{i}^{m} \lambda_{j}^{n}\right\rangle$ on the subsystems A and B translate into joint measurement of spatial modes shown on the OAM Bloch sphere and polarization states shown on the Poincaré sphere. These measurements are graphically depicted in Fig. 28(a) for a vector mode with $\ell_{1}=-\ell_{2}=1, \alpha=1 / 2$, and $\varphi=0$ in Eq. (51). Similarly, Fig. 28(b) shows graphically the outcomes of the 36 measurements for a scalar beam with $\ell_{2}=-1, \alpha=0$, and $\varphi=0$. One then computes the expectation values $\Omega_{m n}$ and obtains the density matrices shown in Figs. 28(c) and 28(d). Note that the reconstructed density matrices in Figs. 28(c) and 28(d) using intense laser beams are identical to those corresponding to the two-photon hybrid quantum states shown earlier in Fig. 19. It is owing to this resemblance to quantum entanglement that vector beams are coined nonquantum entangled states. With regards to a QST, nonquantum entangled states provide useful insights in the measurement procedure for the expectation values of the density matrix without having to work with single photons.

\subsection{Measurement in a Classical QST}

A QST requires the experimentalist to perform a series of tomographic measurements. We have shown in the quantum cases of single and bi-qubit states that these measurements can be realized using polarization optics or holographic filters. When transferring the principles of QST from the quantum to the classical world and applying them to vector modes, we have shown that the tomographic measurements now required projections on both space and polarization degrees of freedom [94,201]. It thus is interesting to examine how these are practically performed in the laboratory. Let us start with polarization projections.

Recall that measurements of the Pauli matrices $\sigma_{i}$ in the polarization basis require projections on various states on the Poincaré sphere. Naturally, one might ask what optical elements are required to perform such projections. Taking advantage of linear optics, one can turn the question around and ask: how can one generate an arbitrary state on the Poincaré sphere? This is because a linear transformation $\mathcal{M}$ that takes $|A\rangle$ to $|B\rangle$ through $\mathcal{M}|A\rangle=|B\rangle$ necessarily takes $|B\rangle$ back to $|A\rangle$ by simply reversing the process. Mathematically, this implies that such a map is unitary and can therefore be reversed by applying the adjoint transformation $\mathcal{M}^{\dagger}$ :

$$
\mathcal{M}^{\dagger}|B\rangle=\mathcal{M}^{\dagger} \mathcal{M}|A\rangle=|A\rangle,
$$


given that unitarity implies $\mathcal{M}^{\dagger} \mathcal{M}=\mathbb{1}$. Thus, if we know how to linearly map state $|A\rangle$ to $|B\rangle$ (preparation), reversing the transformation takes us from $|B\rangle$ to $|A\rangle$ : detection. Here is how this is used in the case of polarization.

First we need a state on the Poincare sphere. This will be our reference state. Let us take it to be the horizontally polarized state $|H\rangle$. Motion on the Poincaré sphere can be achieved through a series of rotations, as shown in Fig. 2. This is achieved using two polarizations optics: a quarter-wave plate $\left(\hat{R}_{\lambda / 4}\right)$ and a half-wave plate $\left(\hat{R}_{\lambda / 2}\right)$. Assuming a 0 angle along the horizontal direction, the matrix representations of these two optics are

$$
\begin{gathered}
\hat{R}_{\lambda / 4}(\xi)=\exp (-i \pi / 4)\left(\begin{array}{cc}
\cos ^{2}(\xi)+i \sin ^{2}(\xi) & (1-i) \sin (2 \xi) / 2 \\
(1-i) \sin (2 \xi) / 2 & i \cos ^{2}(\xi)+\sin ^{2}(\xi)
\end{array}\right), \\
\hat{R}_{\lambda / 2}(\xi)=\exp (-i \pi / 2)\left(\begin{array}{cc}
\cos (2 \xi) & \sin (2 \xi) \\
\sin (2 \xi) & -\cos (2 \xi)
\end{array}\right) .
\end{gathered}
$$

By using the two waveplates in tandem, one can generate arbitrary states on the Poincaré sphere, some of which are shown in Table 5. Thus, one can produce arbitrary states on the surface of the Poincaré sphere using a polarizer to fix the reference (in our example, horizontally polarized light), and a combination of a quarter- and half-wave plates. Given that the generation setup is known from Table 5, namely, polarizer-quarter-wave plate-half-wave plate, the setup for the detection is deduced to simply be the reverse: half-wave plate-quarter-wave plate-polarizer.

We use a similar approach to perform projections on the spatial degree of freedom, extensively studied using liquid crystal based spin-orbit converters [202-205]. To manipulate the spatial degree of freedom in our case, we make use of spin-orbit coupling with a geometric phase element in the form of our metasurface, as discussed earlier [93]. As the name implies, the state produced has coupled spatial and polarization degrees of freedom, coupling that also depends on the initial polarization state. First, we need to decouple the two degrees of freedom; that means we need to generate/detect spatial modes independently of their polarization. Given that spin-orbit coupling changes the polarization state, we can fix a reference before and after the geometric phase element. Just as in the previous demonstration with polarization, this can be achieved by using a polarizer.

Next, we select our spatial mode by accounting for the transformation of our geometric phase element, for example: $|\ell\rangle|R\rangle \rightarrow|\ell-2 m\rangle|L\rangle$ and $|\ell\rangle|L\rangle \rightarrow|\ell+2 m\rangle|R\rangle$, where $m$ is the topological charge of the plate. Assume that we start with an arbitrarily polarized Gaussian beam $(\ell=0)$. The selection of spatial mode is as described in the following four steps.

1. Use the polarizer to set your desired reference (our choice in the example is horizontal):

$$
a|0\rangle|R\rangle+b|0\rangle|L\rangle \stackrel{\text { polariser }}{\longrightarrow}|0\rangle|R\rangle+|0\rangle|L\rangle=|0\rangle|H\rangle
$$

Table 5. Transformation of an Input Horizontally Polarized State on the Surface of the Poincaré Sphere Using Wave Plates

\begin{tabular}{ccccccc}
\hline$\hat{R}_{\lambda / 4}$ & $\xi_{1}=-\pi / 4$ & $\xi_{1}=-\pi / 4$ & $\xi_{1}=0$ & $\xi_{1}=0$ & $\xi_{1}=\pi / 4$ & $\xi_{1}=\pi / 4$ \\
\hline$\hat{R}_{\lambda / 2}$ & $\xi_{2}=0$ & $\xi_{2}=0$ & $\xi_{2}=0$ & $\xi_{2}=\pi / 4$ & $\xi_{2}=\pi / 8$ & $\xi_{2}=-\pi / 8$ \\
\hline State & $|R\rangle$ & $|L\rangle$ & $|H\rangle$ & $|V\rangle$ & $|D\rangle$ & $|A\rangle$ \\
\hline
\end{tabular}


2. Introduce spin-orbit coupling with the $J$-plate (or equivalent element):

$$
|0\rangle|R\rangle+|0\rangle|L\rangle \stackrel{J \text {-plate }}{\longrightarrow}|-2 m\rangle|L\rangle+|2 m\rangle|R\rangle .
$$

3. Use polarization rotators to control the coupled space-polarization basis states:

$$
|-2 m\rangle \hat{R}_{\lambda / 2}\left(\xi_{2}\right) \hat{R}_{\lambda / 4}\left(\xi_{1}\right)|L\rangle+|2 m\rangle \hat{R}_{\lambda / 2}\left(\xi_{2}\right) \hat{R}_{\lambda / 4}\left(\xi_{1}\right)|R\rangle .
$$

Examples of vector modes produced for different orientations of the half- and quarter-wave plates are shown in Table 6.

4. Select a particular state with a linear polarizer at the reference orientation (we use horizontal). In our case, the states selected after the polarizer are as shown in Table 7.

Exploiting the linearity of our wave plates and the fact that the reference states (horizontally polarized) at both ends of the transformations remain unchanged, the detection procedure is exactly as highlighted above, but in reverse. Note that in the above, we have purposely omitted normalization constants to avoid cluttering the description.

In the case of the tomographic measurement of a state of classically non-separable light, projections on the space and polarization degrees of freedom are performed in tandem using the optics described above. The reverse operations applied to perform the tomographic projections can be expressed as an inner product measurement between an input state $\psi(\mathbf{r})$ and a post-selected scalar projection with spatial pattern $u(r)$ and polarization $\hat{s}_{i}$. After passing through the various optics, the resulting field is given by the product $\psi(\mathbf{r}) \cdot u^{*}(r) \hat{s}_{i}^{\dagger}$. By Fourier transforming the output and probing the beam on the optical axis (that is, setting the spatial frequency to 0 ), one can express each tomographic projection as an inner product measurement between the incident

Table 6. Spin-Orbit States Produced through Polarization Control

\begin{tabular}{llr}
\hline$\hat{R}_{\lambda / 2}$ & \multicolumn{1}{c}{$\hat{R}_{\lambda / 4}$} & State Produced \\
\hline$\xi_{2}=0$ & $\xi_{1}=\pi / 4$ & $|-2 m\rangle|H\rangle+|2 m\rangle|V\rangle$ \\
\hline$\xi_{2}=0$ & $\xi_{1}=-\pi / 4$ & $|-2 m\rangle|V\rangle+|2 m\rangle|H\rangle$ \\
\hline$\xi_{2}=0$ & $\xi_{1}=0$ & $|-2 m\rangle|L\rangle+|2 m\rangle|R\rangle$ \\
\hline$\xi_{2}=\pi / 4$ & $\xi_{1}=0$ & $|-2 m\rangle|L\rangle-|2 m\rangle|R\rangle$ \\
\hline$\xi_{2}=\pi / 8$ & $\xi_{1}=-\pi / 4$ & $|-2 m\rangle|D\rangle-i|2 m\rangle|A\rangle$ \\
\hline$\xi_{2}=-\pi / 8$ & $\xi_{1}=-\pi / 4$ & $|-2 m\rangle|D\rangle+i|2 m\rangle|A\rangle$ \\
\hline
\end{tabular}

Table 7. Post-Selected Horizontally Polarized State

\begin{tabular}{lc}
\hline State before Polarizer & State after Polarizer \\
\hline$|-2 m\rangle|H\rangle+|2 m\rangle|V\rangle$ & $|-2 m\rangle$ \\
\hline$|-2 m\rangle|V\rangle+|2 m\rangle|H\rangle$ & $|2 m\rangle$ \\
\hline$|-2 m\rangle|L\rangle+|2 m\rangle|R\rangle$ & $|-2 m\rangle+|2 m\rangle$ \\
\hline$|-2 m\rangle|L\rangle-|2 m\rangle|R\rangle$ & $|-2 m\rangle-|2 m\rangle$ \\
\hline$|-2 m\rangle|D\rangle-i|2 m\rangle|A\rangle$ & $|-2 m\rangle-i|2 m\rangle$ \\
\hline$|-2 m\rangle|D\rangle+i|2 m\rangle|A\rangle$ & $|-2 m\rangle+i|2 m\rangle$ \\
\hline
\end{tabular}


beam and the post-selected state (see Refs. [130,143,148,206] for an overview of this approach):

$$
\left|\int \mathrm{d} r \psi(\mathbf{r}) \cdot u^{*}(r) \hat{s}_{i}^{\dagger}\right|^{2}=\left|\left\langle u, s_{i} \mid \psi\right\rangle\right|^{2}
$$

\subsection{Bell Measurement with Classical Light}

One test of "quantumness" is a violation of a Bell's inequality [207], in optics the adapted CHSH inequality [163], devised to test for local hidden variables that would underpin the seemingly counterintuitive predictions of quantum mechanics. One of the main assertions of quantum mechanics is that correlations between two entangled particles persist, irrespective of the physical separation between the particles. This statement is rather profound as it rules out entanglement as an interaction, but rather embraces Einstein's description of "spooky action at a distance." It is to rule out (or confirm) a theory based on local hidden variables that the Bell parameter, $S$, places a bound on local realism. In the case of two-qubit entanglement, $S$ is defined as

$$
S=E\left(\theta_{1}, \theta_{2}\right)-E\left(\theta_{1}, \theta_{2}^{\prime}\right)+E\left(\theta_{1}^{\prime}, \theta_{2}\right)+E\left(\theta_{1}^{\prime}, \theta_{2}^{\prime}\right),
$$

where $\theta_{i}$ and $\theta_{i}^{\prime}$ are different angle projections and admits an upper bound $|S| \leq 2$ for any classical hidden variable theory. However, quantum entangled states are allowed to violate this bound, with maximally entangled states reaching a maximum value of $|S|=2 \sqrt{2}$ in two dimensions.

The adapted CHSH inequality was originally devised for quantum states entangled in polarization, considering a source emitting pairs of entangled photon in opposite directions. To perform the measurement, photon $\mathrm{A}$ is passed through a rotating polarizer with transmission axis at $\theta_{1}$, while photon $\mathrm{B}$ is passed through a similar polarizer with transmission axis at $\theta_{2}$. The photon correlation functions $E\left(\theta_{1}, \theta_{2}\right)$ are given by

$$
E\left(\theta_{1}, \theta_{2}\right)=\frac{I\left(\theta_{1}, \theta_{2}\right)+I\left(\theta_{1}^{\perp}, \theta_{2}^{\perp}\right)-I\left(\theta_{1}^{\perp}, \theta_{2}\right)-I\left(\theta_{1}, \theta_{2}^{\perp}\right)}{I\left(\theta_{1}, \theta_{2}\right)+I\left(\theta_{1}^{\perp}, \theta_{2}^{\perp}\right)+I\left(\theta_{1}^{\perp}, \theta_{2}\right)+I\left(\theta_{1}, \theta_{2}^{\perp}\right)},
$$

where $\theta_{i}^{\perp}=\theta_{i}+\pi / 2$, and $I\left(\theta_{1}, \theta_{2}\right)$ is the probability of measuring the two photons in coincidence, when polarizers $\mathrm{A}$ and $\mathrm{B}$ have their transmission axes at $\theta_{1}$ and $\theta_{2}$, respectively. The violation is indicative of how entangled the state is, as dictated by the non-separability of the degrees of freedom in which the entanglement is expressed. In Fig. 29 we show the result of such a measurement on biphotons entangled in OAM.

An analogous measurement can be performed on the non-separable vector beams. Here, $I\left(\theta_{1}, \theta_{2}\right)$ does not represent a probability of coincidence measurements, but rather intensity measurements on the classical beam. Recall the analogy between the Poincaré and Bloch spheres. A vector beam is a hybrid state of polarization and OAM. By mapping polarization states on the Poincare sphere to OAM states on the Bloch sphere, one deduces the classical analogy of $I\left(\theta_{1}, \theta_{2}\right)$; namely, it is the joint probability of projecting the vector mode onto a linear polarization state on the equator of the Poincare sphere, $\left|\theta_{1}\right\rangle$, parameterized as follows: $\left|\theta_{1}\right\rangle=$ $\left(|R\rangle+\exp \left(-2 i \theta_{1}\right)|L\rangle\right) / \sqrt{2}$. The angle $\theta_{2}$ can be viewed as describing a state $\left|\theta_{2}\right\rangle$ on the equator of the Bloch sphere, such that $\left|\theta_{2}\right\rangle=\left(|\ell\rangle+\exp \left(2 i \theta_{2}\right)|-\ell\rangle\right) / \sqrt{2}$. For an arbitrary vector vortex mode described by Eq. (56), one then obtains

$$
I\left(\theta_{1}, \theta_{2}\right)=\left|\left\langle\theta_{1}, \theta_{2} \mid \Psi\right\rangle\right|^{2}=\frac{1+\sin \theta \cos \left(2 \theta_{1}-2 \theta_{2}+\phi\right)}{4} .
$$


From Eqs. (65) and (66), one can show that the Bell parameter reaches a maximum values of $2 \sqrt{2}$ for $\theta_{1}=0, \theta_{2}=\pi / 8$ and $\theta_{i}^{\prime}=\theta_{i}+\pi / 4$. Theoretical simulations of $I\left(\theta_{1}, \theta_{2}\right)$ for our "classically entangled" vector beams are shown in Fig. 30. This works because we are measuring the non-separability of the state, which is not an intrinsically quantum property.

What we have shown in this and the previous sections is that there is no difference between the classical non-separable state and a quantum entangled state insofar as a QST or Bell violation is concerned, so long as we replace the concept of two photons with two degrees of freedom. This means that all biphoton qubit QSTs can be demonstrated pedagogically with vector beams, the core message of this tutorial. We will now demonstrate how to actually do this in the laboratory in the next section.

\subsection{Experimental Demonstration}

In this section we wish to set up an experiment and perform the required measurements to demonstrate the theory we have covered so far. Importantly, we wish to do it on purely classical light. We wish to proceed as if we had two photons entangled in some degree of freedom, but rather than a quantum experiment we will perform the tests with a much simpler classical experiment. Both quantum-state tomographic measurements and the CHSH Bell-like inequality measurements can be achieved with the experimental configuration detailed in Fig. 31, dividing the process into two core parts: the generation of the non-separable classical states and projection of these states into the proper bases for QST and for violation of a Bell-type inequality. We wish to compute the density matrix, degree of entanglement, fidelity, and the $S$ parameter. Further, we wish to control the mode at the generation step to illustrate that the entanglement can be varied very easily, as well as changed from one subspace to another with simple optics. Here we will use the term entanglement, whereas it should

\section{Figure 29}

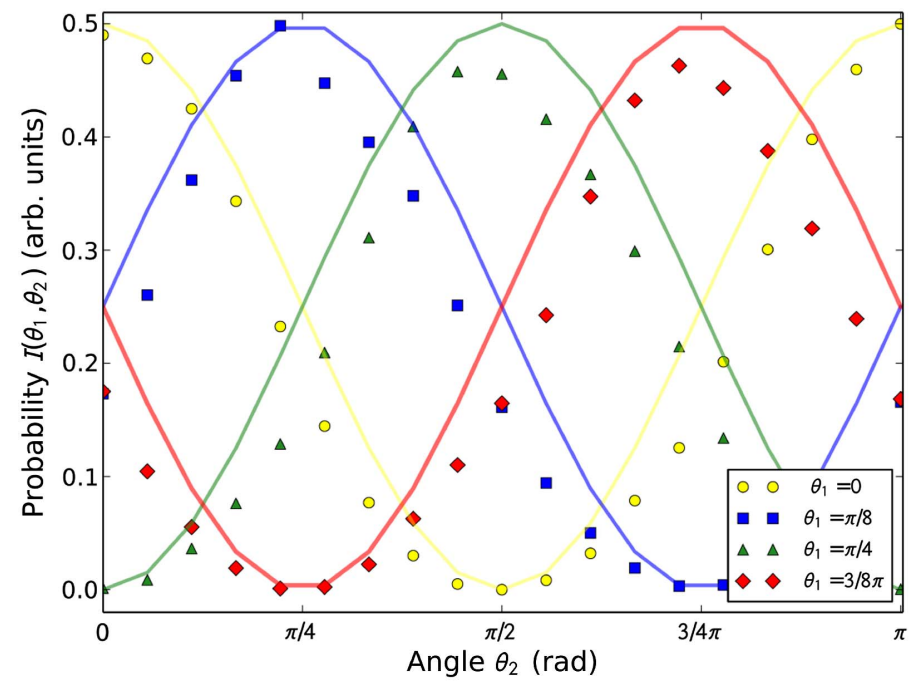

Bell measurement with spatially separated photon pairs generated from a spontaneous parametric downconversion source. Local measurements were performed using spatial analyzers that project each photon onto superposition states defined on the equator of the Bloch sphere. The projections were mapped onto the states $\left(|\ell\rangle+\exp \left(-2 i \theta_{1}\right) \mid-\right.$ $\ell\rangle) / \sqrt{2}$ and $\left(|\ell\rangle+\exp \left(2 i \theta_{2}\right)|-\ell\rangle\right) / \sqrt{2}$. Subsequently, the signals of the projected photons were measured in coincidence with an amplitude proportional to $I\left(\theta_{1}, \theta_{2}\right)$. The high visibility of the amplitude variation is indicative of non-local interactions between the spatially separated photons [208]. 
now be understood that we are referring to non-separability of the vector state, a local entanglement, as a proxy for what one would see with true quantum entanglement between two photons.

In the generation step we need to prepare vector beams of the form given by Eq. (51). We will control the parameters to control the degree of non-separability, moving freely around the HOPS. Moreover, we will switch from one HOPS to another by use of geometric phase optics, as explained earlier. The essential task is that the outcome is some desired state on a HOPS that can then be analyzed.

In our example setup we used a green laser ( $\lambda=532 \mathrm{~nm}$, Verdi G5, Coherent) that was directed to the setup through the fiber and collimated with a collimation package (F220 HPC-532, Thorlabs), yielding a Gaussian beam $(\ell=0)$ of radius $1.05 \mathrm{~mm}$. A demagnification of the beam radius is needed to keep the beam within the dimensions of the geometric optic, $J_{1}$. The new beam radius of $175 \mu \mathrm{m}$ was obtained with a telescope formed by lenses $\mathrm{L}_{1}(f=300 \mathrm{~mm})$ and $\mathrm{L}_{2}(f=300 \mathrm{~mm})$. The output was then vertically polarized with $P_{0}$. Waveplates $\mathrm{HWP}_{0}$ and $\mathrm{QWP}_{0}$ allowed manipulation of the polarization state incident on $J_{1}$, generating the desired state on the HOPS.

The HOPS states were generated by using a nano-structured dielectric spin-to-orbital angular momentum converter of radius $250 \mu \mathrm{m}$ and designed for green light. This optical device, $J_{1}$, correlates the OAM of the vortex beam with the polarization of the incoming light, being able to flip the sign of the OAM charge contribution by illuminating the device with a particular circular polarization state (see section on spin-orbit coupling). The devices used in our tests performed the transformations

$$
\begin{aligned}
& |0\rangle|R\rangle \Rightarrow|-1\rangle|L\rangle, \\
& |0\rangle|L\rangle \Rightarrow|+1\rangle|R\rangle .
\end{aligned}
$$

That is to say, the handedness of the incoming circularly polarized light induces a handedness on the topological charge $\ell$ in the vortex beam at the output. For example, incident with a superposition of circular polarization states results in the generation of

\section{Figure 30}

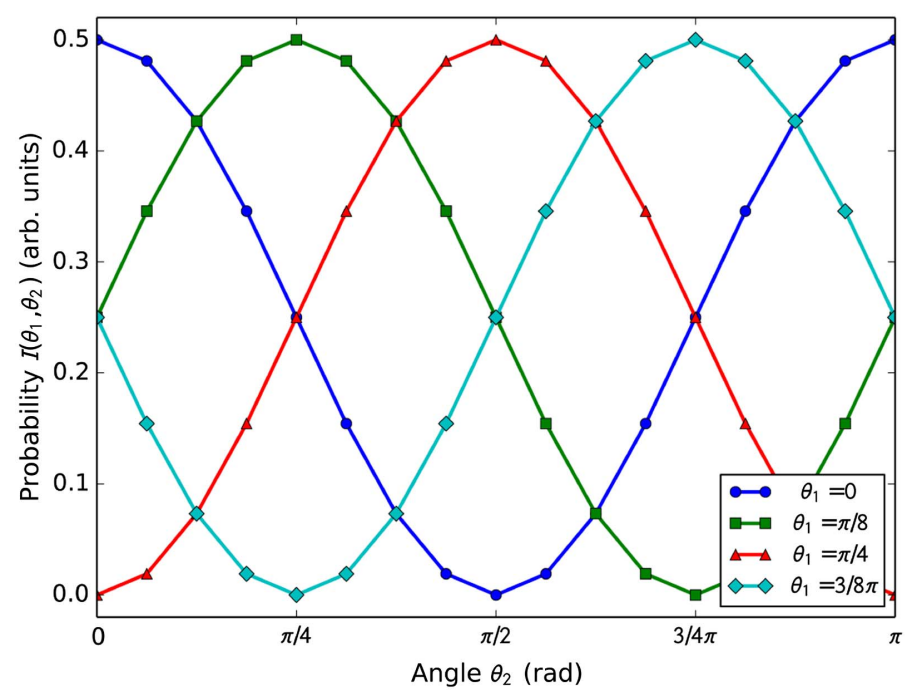

Simulation of Bell measurements on an input vector vortex beam. The angles $\theta_{1}$ and $\theta_{2}$ parameterize the states used to compute the projection. These are polarization and OAM superposition states on the equator of the Poincaré and Bloch spheres, $\left(|R\rangle+\exp \left(-2 i \theta_{1}\right)|L\rangle\right) / \sqrt{2}$ and $\left(|\ell\rangle+\exp \left(2 i \theta_{2}\right)|-\ell\rangle\right) / \sqrt{2}$. 
vector modes as given by Eq. (56) with $\theta=\frac{\pi}{2}$ and $\phi=0$. The degree of non-separability may consequently be made to vary from purely scalar to maximally non-separable. It follows that generation of the HOPS states may be accomplished simply by manipulating the circular polarization weightings of the beam being directed through the geometric phase plate. For example, in the experiment, the laser beam polarization was set to horizontal, using $P_{0}$, allowing full control of the polarization being passed to the phase plate by manipulation of waveplates before $J_{1}$, as shown in the generation step of Fig. 31(a). Accordingly, for the generation of a scalar state, $\mathrm{QWP}_{0}$ placed at $45^{\circ}$ resulted in circularly polarized Gaussian input, which was then converted to a single OAM beam with opposite circular polarization. In the case of generating a classically entangled state, adjusting the angle of the $\mathrm{HWP}_{0}$ (with $\mathrm{QWP}_{0}$ removed) allowed one to tailor the phase difference between the circular polarization superposition comprising the linear states chosen. This circular-polarization superposition thus results in the generation of an OAM superposition once passed through $J_{1}$.

For state projection, we performed a QST and Bell measurement as described earlier. The 36 configurations of the optics required for an over-complete tomographic measurement of the state are listed in Fig. 32. The capital letters R, L, H, V, D, and A refer to right-circular, left-circular, horizontal, vertical, diagonal, and anti-diagonal

\section{Figure 31}
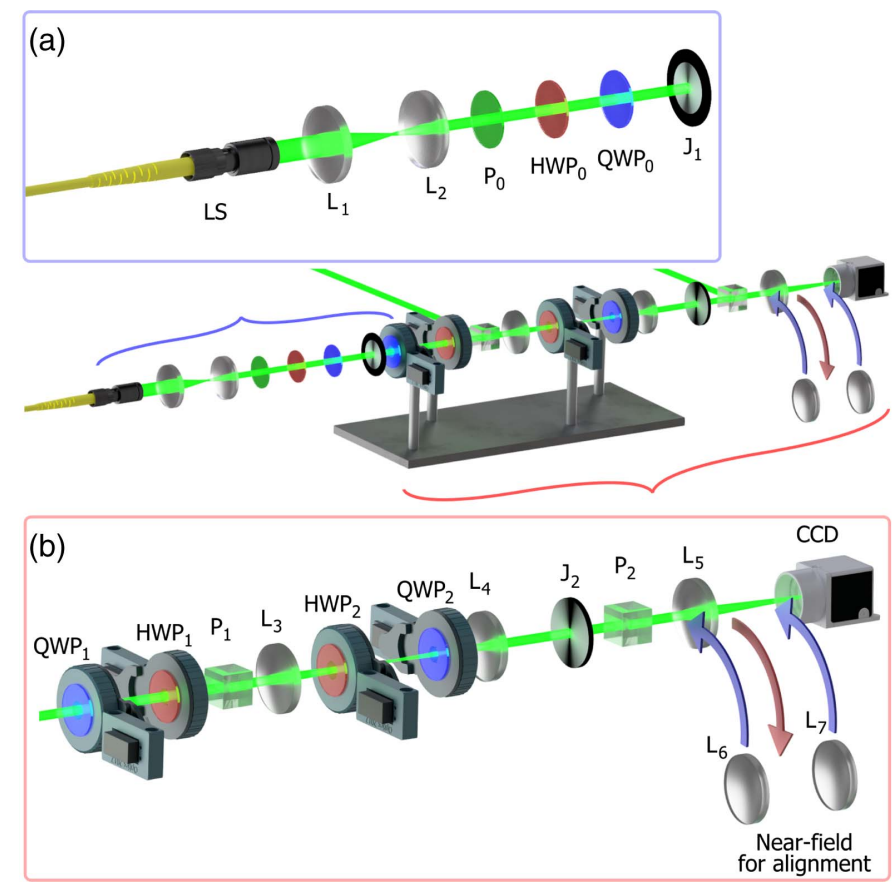

Experimental setup scheme: (a) state generation and (b) automated state projection. LS, laser source (532 nm fiber-coupled light from Verdi G5, Coherent); $\mathrm{L}_{i}$, Fourier lenses; $P_{0,1 \& 2}$, polarizers; $\mathrm{HWP}_{0,1 \& 2}$, half-waveplates; $\mathrm{QWP}_{0,1 \& 2}$, quarter-waveplates; CCD, Chameleon3 CCD camera (Point Grey, FLIR). The waveplates $\mathrm{HWP}_{1 \& 2}$ and $\mathrm{QWP}_{1 \& 2}$ are mounted on roto-flip mounts. The lenses $\mathrm{L}_{1}(f=300 \mathrm{~mm})$ and $\mathrm{L}_{2}(f=50 \mathrm{~mm})$ demagnify the laser beam to match the size of $J_{1}, \mathrm{~L}_{3 \& 4}$ $(f=300 \mathrm{~mm})$ relay the plane of $J_{1}$ onto $J_{2}, \mathrm{~L}_{5}(f=150 \mathrm{~mm})$ Fourier transforms the $J_{2}$ plane to spatially project into the Gaussian mode $(\ell=0)$ or, replacing it by $\mathrm{L}_{6 \& 7}(f=75 \mathrm{~mm})$, the plane of $J_{2}$ is relayed onto the CCD for alignment purposes. Polarizers $P_{1 \& 2}$ are polarizing beam splitters, providing extra output from port orthogonal to the optical axis for convenience during alignment. 
polarizations, respectively. In our experimental realization, the polarization states and OAM states shown in Fig. 32 are related to the angular positions of optical components $\mathrm{QWP}_{1}, \mathrm{QWP}_{1}$ and $\mathrm{HWP}_{2}, \mathrm{QWP}_{2}$, as shown in Fig. 33.

In our experiment we used 3D printed and home-automated optics to make the entire experiment inexpensive and DIY (see the section to follow for the resources on how to do this). First, the combination of waveplates $\mathrm{QWP}_{1}$ and $\mathrm{HWP}_{1}$ (both automated), and the polarizer $P_{1}$ is used to project only the polarization degree of freedom from the input state without affecting the OAM. Accordingly, one is able to select each polarization state shown alongside the rows in Fig. 28(b) just by clicking a button. For example, consider the first row of Fig. 28(b), which corresponds to the first row of Fig. 32. Here the selection of $|R\rangle$ is achieved by placing these waveplates at the angles specified by $R_{1} .|R\rangle$ is then converted to horizontal polarization, resulting in the $|R\rangle$ component of the generated beam being isolated by $P_{1}$ and carrying the unaffected OAM information through.

Projection of the chosen polarization state onto the OAM states illustrated alongside the columns of Fig. 28(b) was then obtained through the use of a second geometric phase optic, $J_{2}$. As the incoming polarization controls the OAM states generated [see Eq. (68)], reversing the process can result in a projective measurement.

\section{Figure 32}

\begin{tabular}{c|c|c|c|c|c|c} 
& $\left|\mathrm{R}_{2}\right\rangle$ & $\left|\mathrm{L}_{2}\right\rangle$ & $\left|\mathrm{H}_{2}\right\rangle$ & $\left|\mathrm{V}_{2}\right\rangle$ & $\left|\mathrm{D}_{2}\right\rangle$ & $\left|\mathrm{A}_{2}\right\rangle$ \\
\hline$\left|\bigcirc \mathrm{R}_{1}\right\rangle$ & $R_{1} R_{2}$ & $R_{1} L_{2}$ & $R_{1} H_{2}$ & $R_{1} V_{2}$ & $R_{1} D_{2}$ & $R_{1} A_{2}$ \\
\hline$\left|\bigcirc \mathrm{L}_{1}\right\rangle$ & $L_{1} R_{2}$ & $L_{1} L_{2}$ & $L_{1} H_{2}$ & $L_{1} V_{2}$ & $L_{1} D_{2}$ & $L_{1} A_{2}$ \\
\hline$\left.\longleftrightarrow \mathrm{H}_{1}\right\rangle$ & $H_{1} R_{2}$ & $H_{1} L_{2}$ & $H_{1} H_{2}$ & $H_{1} V_{2}$ & $H_{1} D_{2}$ & $H_{1} A_{2}$ \\
\hline$\left|\downarrow V_{1}\right\rangle$ & $V_{1} R_{2}$ & $V_{1} L_{2}$ & $V_{1} H_{2}$ & $V_{1} V_{2}$ & $V_{1} D_{2}$ & $V_{1} A_{2}$ \\
\hline$\left|\nearrow \mathrm{D}_{1}\right\rangle$ & $D_{1} R_{2}$ & $D_{1} L_{2}$ & $D_{1} H_{2}$ & $D_{1} V_{2}$ & $D_{1} D_{2}$ & $D_{1} A_{2}$ \\
\hline$\left|\backslash \mathrm{A}_{1}\right\rangle$ & $A_{1} R_{2}$ & $A_{1} L_{2}$ & $A_{1} H_{2}$ & $A_{1} V_{2}$ & $A_{1} D_{2}$ & $A_{1} A_{2}$
\end{tabular}

Thirty-six angular arrangements of the polarization optics. The subscript- 1 and subscript-2 terms indicate the polarization states and the OAM states created with the first and second pairs of QWPs and HWPs, respectively.

\section{Figure 33}

\begin{tabular}{c|c||c|c|c|c|c} 
& $\left|\bigcirc \mathrm{R}_{1}\right\rangle$ & $\left|\bigcirc \mathrm{L}_{1}\right\rangle$ & $\left|\longrightarrow \mathrm{H}_{1}\right\rangle$ & || $\left.\mathrm{V}_{1}\right\rangle$ & $\left|\mathrm{D}_{1}\right\rangle$ & $\left|\mathrm{A}_{1}\right\rangle$ \\
\hline $\mathbf{Q W P}_{\mathbf{1}}$ & $-\pi / 4$ & $\pi / 4$ & 0 & 0 & $\pi / 4$ & $\pi / 4$ \\
\hline $\mathbf{H W P}$ & 0 & 0 & 0 & $\pi / 4$ & $\pi / 8$ & $-\pi / 8$ \\
& $\left|\mathrm{R}_{2}\right\rangle$ & $\left|\mathrm{L}_{2}\right\rangle$ & $\left|\mathrm{H}_{2}\right\rangle$ & $\left|\mathrm{V}_{2}\right\rangle$ & $\left|\mathrm{D}_{2}\right\rangle$ & $\left|\mathrm{A}_{2}\right\rangle$ \\
\hline $\mathbf{H W P}_{2}$ & 0 & 0 & 0 & $\pi / 4$ & $\pi / 8$ & $-\pi / 8$ \\
\hline $\mathbf{Q W P}_{2}$ & $\pi / 4$ & $-\pi / 4$ & 0 & 0 & $-\pi / 4$ & $-\pi / 4$
\end{tabular}

In our experimental realization, the indicated angular positions in radians of $\mathrm{QWP}_{1}$, $\mathrm{QWP}_{1}$ and $\mathrm{QWP}_{2}, \mathrm{HWP}_{2}$ allow to generate the desired polarization states. The subscript-1 and subscript-2 terms indicate the polarization states and the OAM states created with the first and second pairs of QWPs and HWPs, respectively. 
Specifically, by transforming the polarization state after $P_{1}$ with the automated rotation of $\mathrm{HWP}_{2}$ and $\mathrm{QWP}_{2}$, the OAM mode experienced by the polarization state traversing the second geometric phase optic may also be altered as governed by the selection rules in Eq. (68). With only $J_{2}$, however, the mode experienced is vectoral in nature and, thus, by placing $P_{2}$ afterward, the correct projective OAM mode superposition may be construed as seen by the spatial modes in the HOPS. Capturing the outcome with the $\mathrm{CCD}$ camera in the Fourier plane after $\mathrm{L}_{5}$ $(f=150 \mathrm{~mm})$ and measuring the on-axis intensity then allows the weighting for each projection to be measured according to the rotational angles listed in Fig. 32.

For example, if we consider a scalar mode at the input of our automated state projection section, such as $|\ell=-1\rangle|L\rangle$, we will have a horizontally polarized state $|\ell=-1\rangle|H\rangle$, with the OAM contribution still intact after $P_{1}$, independently of the chosen polarization projection. This state is transformed by $\mathrm{HWP}_{2}$ and $\mathrm{QWP}_{2}$ with the angles at the configurations described as $V_{2}$, converting it into vertical polarization $\frac{1}{\sqrt{2}}|\ell=-1\rangle\{|R\rangle-|L\rangle\}$. It is then trivial to see that, by directing $|V\rangle$ through $J_{2}$ and $P_{2}$, the OAM information carried by the initial $|R\rangle$ subsequently experiences OAM transformation $(|\ell=-1\rangle \rightarrow|\ell=0\rangle$ ), following the selection rules of Eq. (68), retaining only the Gaussian mode contribution after spatially filtering the resulting intensity profile at the Fourier plane.

Alignment of the system was simplified by imaging $J_{1}$ onto $J_{2}$. Replacement of $\mathrm{L}_{5}$ with $\mathrm{L}_{6}$ and $\mathrm{L}_{7}$ allowed the geometric phase optics to be further imaged onto the $\mathrm{CCD}$ such that the positions may be adjusted to achieve a centered superposition of the singularities. Figures 34-37 show the experimental data of example QST measurements and the resulting density matrices when choosing various input states on the HOPS. In the QST data, each row corresponds to a particular polarization

\section{Figure 34}

(a)

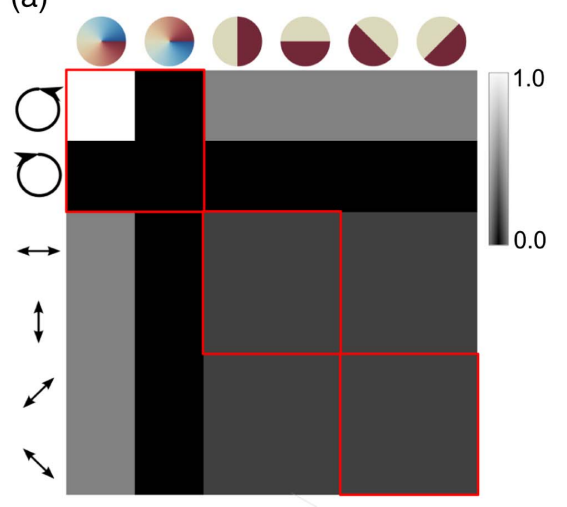

(c)

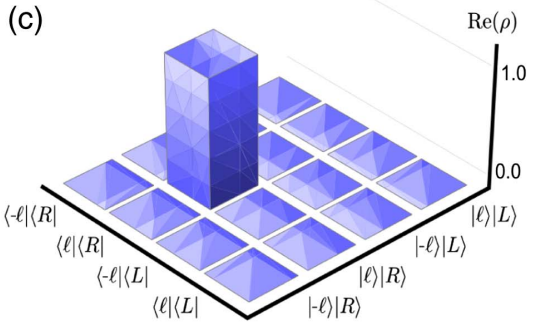

(b)

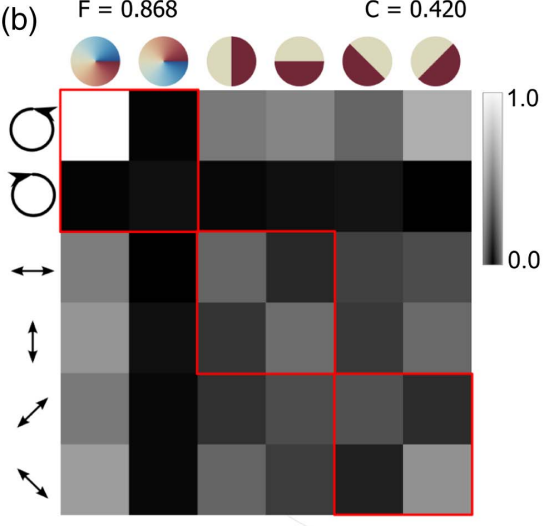

(d)

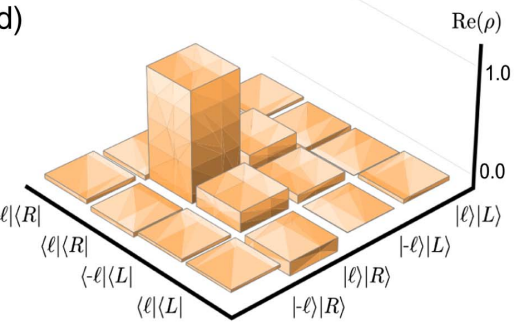

Quantum-state tomography measurements (a) theory and (b) experiment, with resulting density matrices in (c) and (d), respectively, for a scalar mode of the form $|\psi\rangle_{0}=|\ell=1\rangle|R\rangle$. 


\section{Figure 35}

(a)

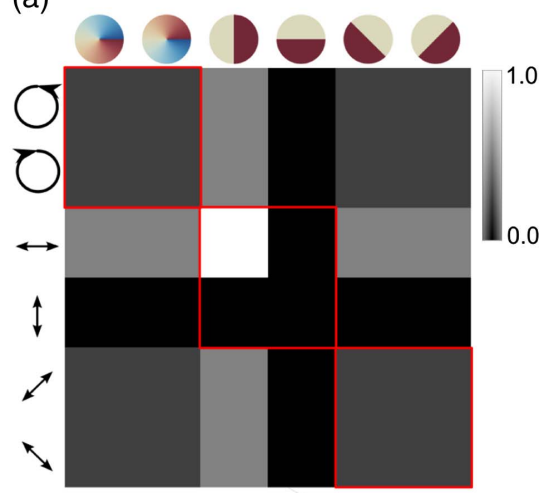

(c)

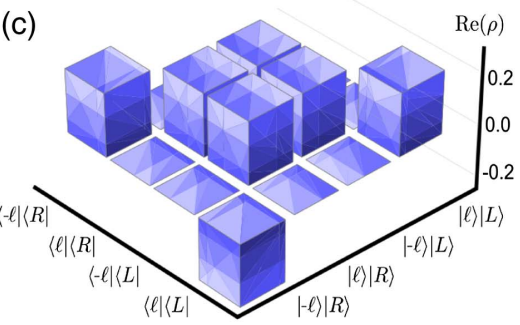

(b)

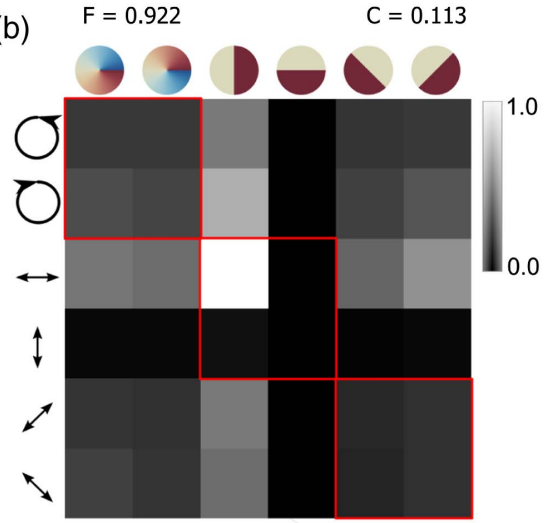

(d)

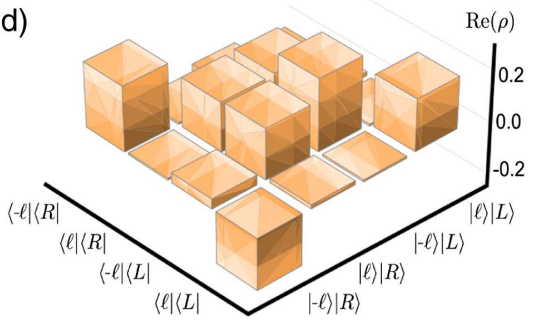

Quantum-state tomography measurements (a) theory and (b) experiment, with resulting density matrices in (c) and (d), respectively, for a horizontally polarized mode of the form $|\psi\rangle_{0}=(|\ell=1\rangle+|\ell=-1\rangle)|H\rangle$.

\section{Figure 36}

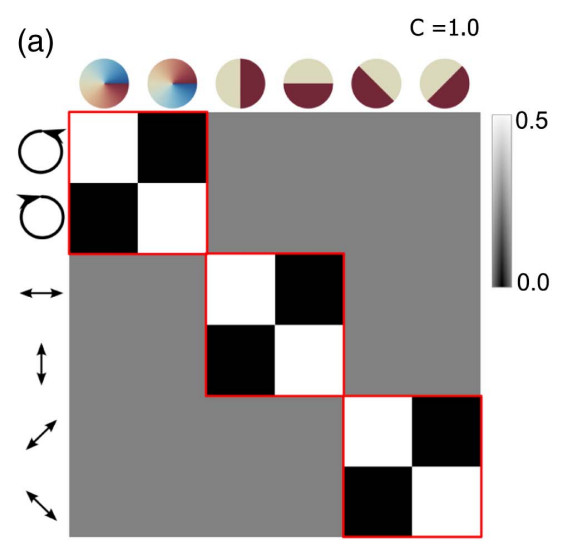

(b)

$\mathrm{F}=0.928$

$C=0.929$

(b)
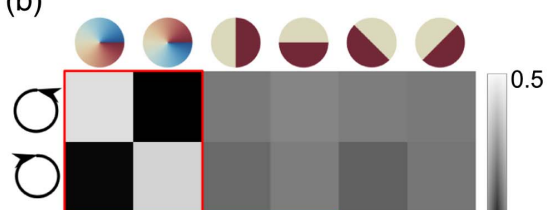

(c)
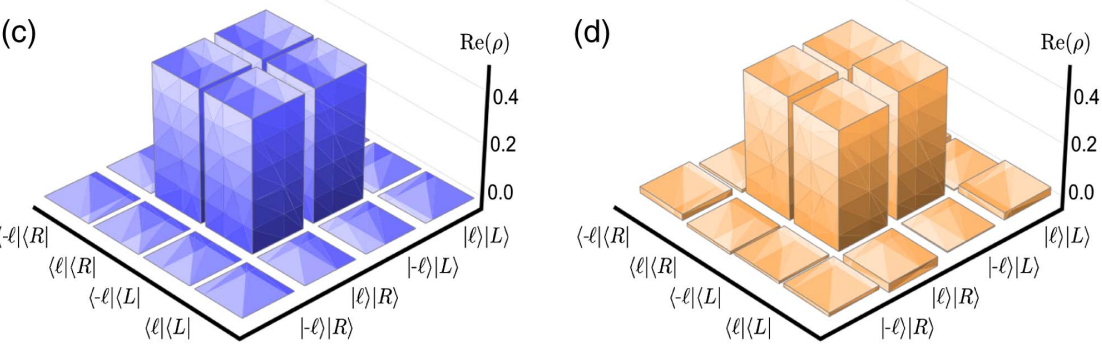

Quantum-state tomography (a) theory and (b) experiment, with resulting density matrices in (c) and (d), respectively, for a vector mode of the form $|\psi\rangle_{0}=\frac{1}{\sqrt{2}}(|\ell=1\rangle|R\rangle+|\ell=-1\rangle|L\rangle)$. 
projection, and each column to the OAM degree of freedom projection. For example, the results shown in Figs. 34 and 35 are the reconstructed graphical representations of tomographic projections for a separable scalar mode [Eq. (69)], while the results shown in Figs. 36 and 37 are the reconstructed graphical representation of the tomographic projections for a maximally non-separable vector mode [Eq. (70)], both in agreement with the theoretical simulation. The states are given below:

$$
\begin{gathered}
|\psi\rangle_{\text {scalar }}=|\ell=-1\rangle|L\rangle, \\
|\psi\rangle_{\text {vector }}=\frac{1}{\sqrt{2}}(|\ell=1\rangle|R\rangle+|\ell=-1\rangle|L\rangle) .
\end{gathered}
$$

The CHSH inequality measurement for our hybrid input state requires selecting two polarization projections, $\theta_{1}$ and $\theta_{1}^{\prime}$ of Eq. (65), and two OAM projections, $\theta_{2}$ and $\theta_{2}^{\prime}$, also considering all their orthogonal angle combinations as detailed in Eq. (66). The first degree of freedom that is projected is the spin, and it is performed by fixing $\mathrm{QWP}_{1}$ and rotating $\mathrm{HWP}_{1}$, selecting the proper polarization angle, i.e., $\theta_{1}$ of Eq. (66). Then, the OAM degree of freedom is projected, but, in this case, also by using polarization control elements (fixing $\mathrm{QWP}_{2}$ and rotating $\mathrm{HWP}_{2}$ ), and decoding its spatial degree of freedom with $J_{2}$. In this case, the OAM state is correlated with the previously selected polarization state, being able to project it after the second polarizer $\left(P_{2}\right)$, by measuring also the Gaussian mode $(\ell=0)$ intensity with a CCD camera in the Fourier plane.

The relation between the projection angles $(\theta)$ from Eq. (65) is not arbitrary, and must always fulfill the following condition:

$$
\theta \equiv \theta_{2}-\theta_{1}=\theta_{2}^{\prime}+\theta_{1}^{\prime}=-\theta_{2}-\theta_{1}^{\prime},
$$

\section{Figure 37}

(a)

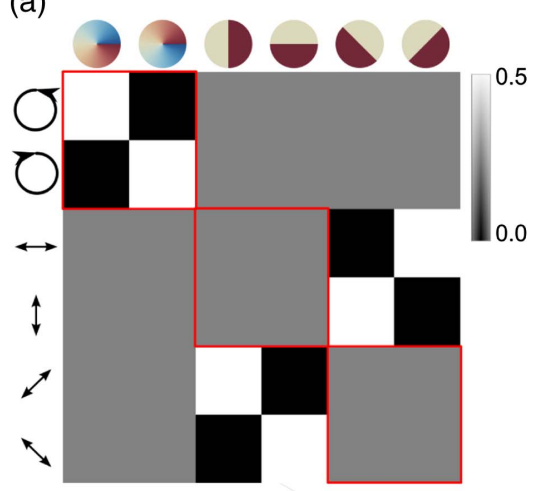

(c)

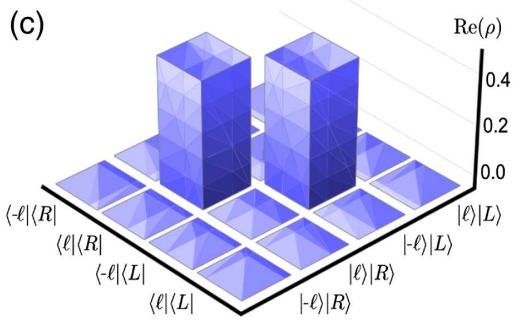

(b)

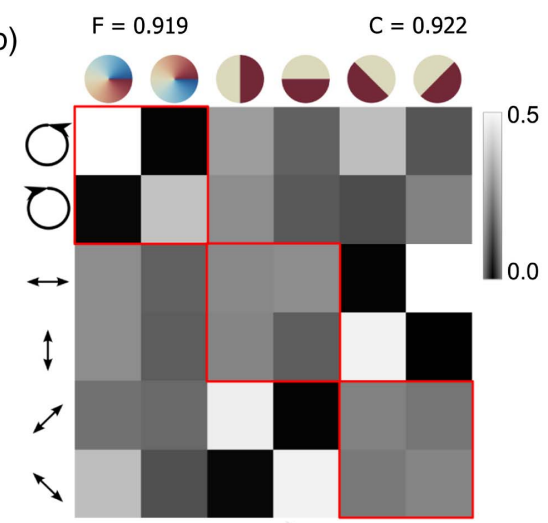

(d)

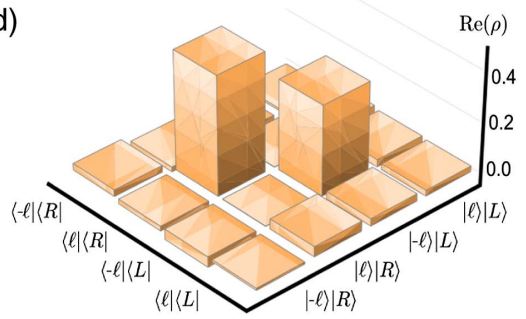

Quantum-state tomography (a) theory and (b) experiment, with resulting density matrices in (c) and (d), respectively, for a vector mode $|\psi\rangle_{0}=\frac{1}{\sqrt{2}}(|\ell=1\rangle|R\rangle+$ $i|\ell=-1\rangle|L\rangle)$. 
giving a maximum value of $S=2 \sqrt{2}$ when choosing $\theta=22.5^{\circ}$ and having a maximally non-separable state, but the angle $\theta$ at which to find the maximum of $S$ increases if the degree of non-separability of the input state decreases [150]. The angles usually chosen to violate the $\mathrm{CHSH}$ inequality are $\theta_{1}=0^{\circ}, \theta_{1}^{\prime}=-45^{\circ}$, $\theta_{2}=22.5^{\circ}$, and $\theta_{2}^{\prime}=67.5^{\circ}$. The high number of projections when considering the orthogonal angles makes the measurement tedious to perform. We show all the curves containing all the necessary values to extract the $S$ parameter value.

As can be seen in Fig. 38, the results of the projection curves used to extract the $S$ parameter of Eq. (65) mimic closely the simulated curves. The normalized intensity is plotted as a function of OAM projection $\theta_{2}$, having different angles of spin projections $\theta_{1}$ under consideration. Experimentally, we performed a full -tomographic measurement for two vector modes input states (i.e., "classically entangled"), as shown in Figs. 38(a) and 38(b), and two scalar modes (i.e., "non-entangled") input states, as shown in Figs. 38(c) and 38(d). As expected, for the two classically entangled states we computed $S=2.60 \pm 0.08$ and $S=2.57 \pm 0.09$, violating the CHSH inequality by more than 7 and 6 standard deviations, respectively. In the case of the two nonentangled input scalar modes, we computed $S=1.42$ and $S=0.10$, respectively, leaving the CHSH inequality unscathed.

In the case of classical light, the experimental error associated to the measured intensity values is not subject to shot noise, as the system operates with a very large number of photons. One would therefore expect a very small standard deviation in the estimated Bell parameters. This was indeed the case in our implementation. However, the $S$ parameter reported above is shown to beat the CHSH inequality by only 7 standard deviations. The reason for this is that we decided to relate the experimental error to the

\section{Figure 38}
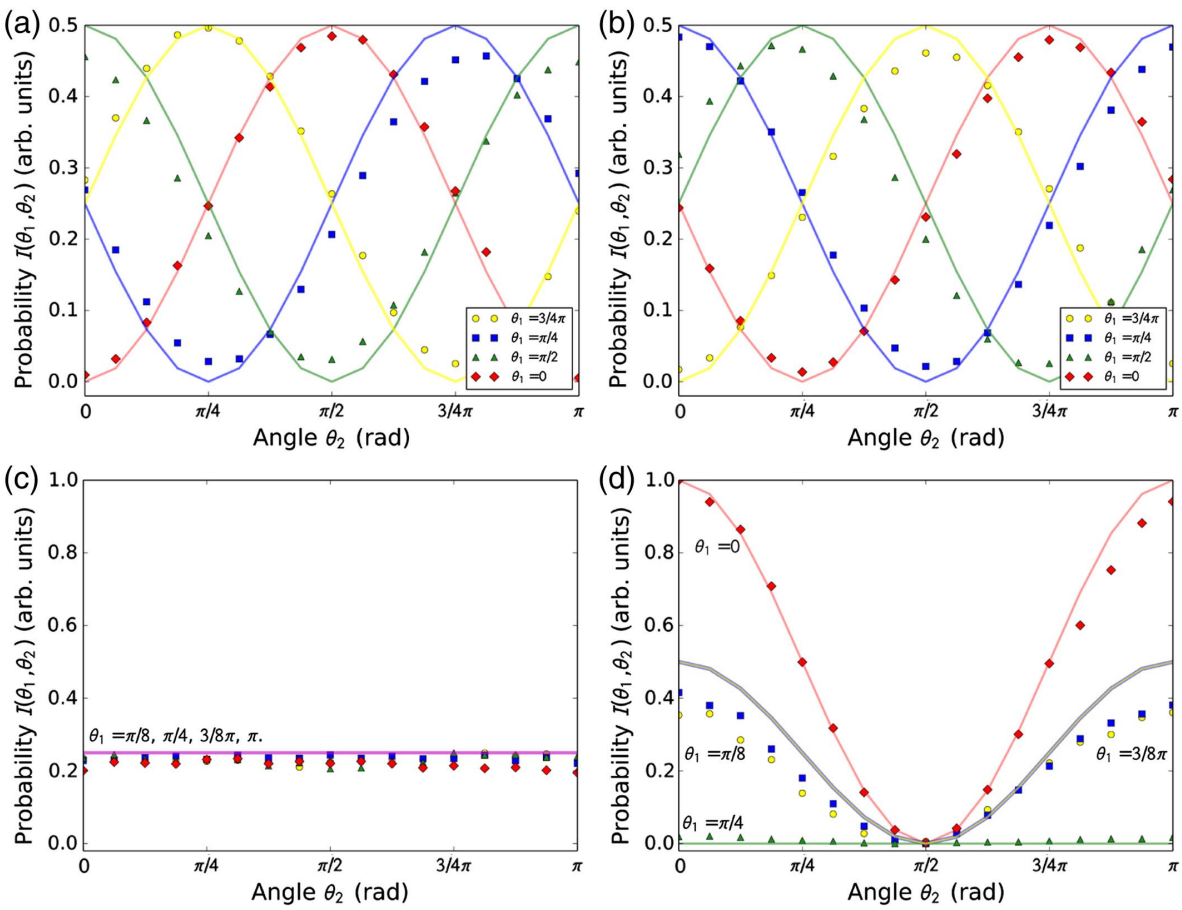

Experimental Bell measurement curves for (a), (b) two "classically entangled" vector modes, and (c), (d) two "non-entangled" scalar modes. The solid lines represent the expected theoretical values, whereas the scattered data points represent the experimentally measured values. 
ability of the roto-flip stages to repeatedly reproduce the full set of angular positions required for a Bell measurement. Consequently, instead of computing the errors based on multiple frames acquired for each angular position of the polarization optics, we allowed instead for only one frame to be acquired at each angular position, repeating the whole procedure for a number of times. This is why the error associated to our estimation of the $S$ parameter is still appreciable, as it now depends on the combined ability of the four roto-flip stages to reproducibly position themselves over multiple runs of the experiment, instead of depending on the small readout noise of the a CCD detector operating with a bright light source and hence at an optimal dynamic range (in the case of our 10 bit CCD camera, the read noise was $\approx 5$ counts, for a full dynamic range of 4096 counts)

The conclusion from these results is that it is possible to perform a QST and associated measurements as if one was operating on a biphoton quantum state. The mathematical formalism means that the process requires no amendments, while physically the results are likewise equivalent: we are measuring the non-separability of the state. This allows the user to perform all tests on intense light beams, a useful approach both for teaching and for research, e.g., initial tests prior to experimenting on single photons.

Perhaps a comment on the limitations of the approach is necessary. While our classical approach accurately mimics the quantum world, it does not replace it. There are many quantum processes for which classical light is not suitable. For example, while the measurement process and probabilities of a quantum key distribution experiment can be simulated (say with the backprojection approach as shown earlier), it is clearly not possible to guarantee the security without true quantum entanglement or true single photons. There are many such examples and it is not necessary to dwell on them all; suffice it to say that what we offer here is an additional resource when performing quantum experiments rather than a replacement for existing quantum approaches.

In the experimental realization outlined in this section we have used home-built 3D printed optical components to allow for easy implementation. As the final part of the tutorial, we now provide the resources for this to be implemented by others.

\section{DIY LABORATORY IMPLEMENTATION}

State-tomography experiments can be a tedious experimental task, involving the painstaking collection of several measurements in order to reconstruct the density matrix of an input state. It may be possible to choose a suitable set of states to conveniently automate these experiments, for instance by using a digital SLM to display computer-generated holograms of an OAM basis. However, SLMs may not be available or may not be suited to the experiment at hand. The alternative is to revert to polarization optics and the meticulous tuning of the angular positions of half-waveplates and quarter-waveplates. In this case, the mere process of manually adjusting each component for each iteration of the experiment becomes an uncomfortable hindrance, which, unless plenty of time is at hand, either prevents the busy experimentalist from obtaining the best set of data, or it makes it hard to appreciate and enjoy the physics, which ends up being diluted over hours and hours of repetitive procedure.

In this section we describe how to produce with a 3D printer inexpensive electromechanical roto-flip stages that allow automation of the angular positioning of polarization optics, and to fully automate the state projection measurements required to reconstruct the density matrix of an unknown input state.

\subsection{D-Printed Roto-Flip Stages}

An obvious advantage of automating an experiment is the resulting speed and reliability of the results, as well as the ability to perform a larger number of incremental 
fine adjustments. To fully automate our polarization-based state-projection measurement, four motorized roto-flip stages were designed, 3D printed, and linked to an automated data-acquisition program written in LabVIEW (available for download, together with the Arduino code and data processing LabVIEW code (Code 1, Ref. [126]; Code 2, Ref. [126]; and Code 3, Ref. [126]), as well as the 3D schematics at Ref. [126]). As the name suggests, each roto-flip stage can both rotate an optical component along the transverse plane, and also act as a flip mount, pivoting $90^{\circ}$ away from the beam path. The rotary motion was achieved with a combination of 3Dprinted spur gears with a 4:1 gear ratio and a stepper motor, obtaining a maximum angular resolution of $0.17^{\circ}$. The pivoting motion was achieved by using a servo. The combination of four motorized roto-flip stages allowed to quickly automate the positioning of two HWPs and two QWPs over 36 angular configurations, performing a full state reconstruction in approximately $2 \mathrm{~min}$, as shown in the video demonstration of our automated tomography system in action, accessible from Ref. [126].

Excellent angular resolution as well as a sturdy and compact design were the main mechanical requirements for the rapid-prototyped angular stages. These requirements can be easily fulfilled by plastic 3D-printed parts and cheap electronics components [209-213]. We used an Ultimaker 2+ 3D printer, loaded with polylactic acid (PLA) filament to print the motion components. The 3D models were designed with Autodesk Inventor. Each stage employed an Arduino Nano microcontroller, to actuate

\section{Figure 39}

(a)
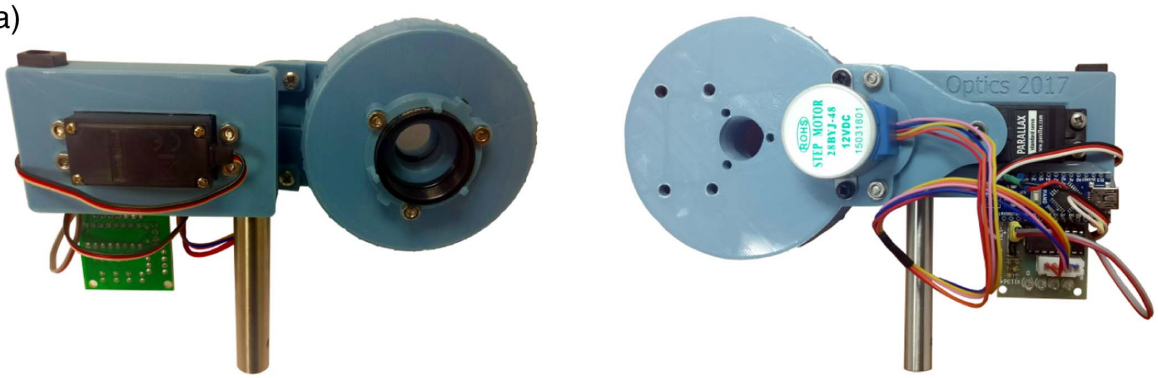

(b)

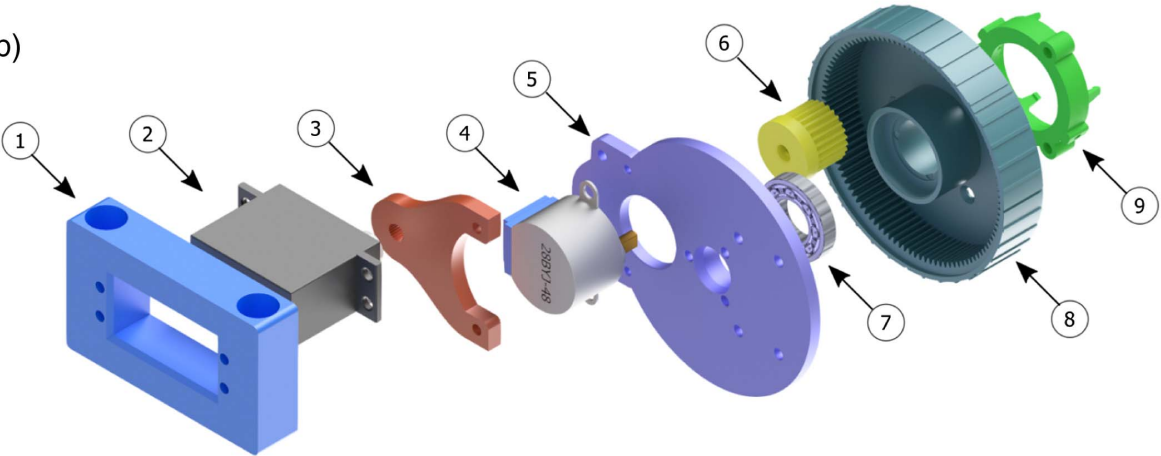

Detailed description of the 3D-printed roto-flip stage. (a) Front and back views; (b) 3D-printed parts assembly. The numbers in the figure indicate the following: (1) roto-flip stage support board; (2) parallax standard servo; (3) servo connection board; (4) 28BYJ-48 stepper motor; (5) back-support board for stepper with ball-bearing slot; (6) small spur gear with 26 teeth; (7) ball bearing (17 $\mathrm{mm}$ inside diameter, $26 \mathrm{~mm}$ outside diameter, $5 \mathrm{~mm}$ race width); (8) big spur gear with 104 teeth and ballbearing slot; (9) clip for SM1 optical components. The various 3D printed parts are assembled by both pressure fitting and by using common M4 screws and nuts. (4), (5), (6), (7), and (8) can be pressure fitted. The screw holes in (1) and (5) were threaded with a tapping tool to complete the assembly. 
the driver (ULN200xx chip) of the stepper motor (28BYJ-48) and operate the servo (Parallax Standard Servo), as well as handling communication with the LabVIEW automation program via the serial port. A detailed representation of the motorized roto-flip mounts is shown in Fig. 39, and the electronics component description is in Fig. 40.

\subsection{Video Demonstration of the Automated State-Tomography System in Action}

A video demonstration of our automated tomography system in action is provided. Both the projected states, as acquired by the CCD camera, and the angular positions of each roto-flip stage are highlighted, as schematically represented by a few extracted frames in Fig. 41. In the video, the system can be seen iterating over the 36 angular positions of the polarization optics (two pairs of quarter- and half-waveplates), which are required to identify an unknown input state. We hope that this video demonstration may facilitate the assimilation of the concepts covered in this tutorial and help the reader appreciate what, from a practical point of view, the tomographic measurement of a state involves.

\section{Figure 40}

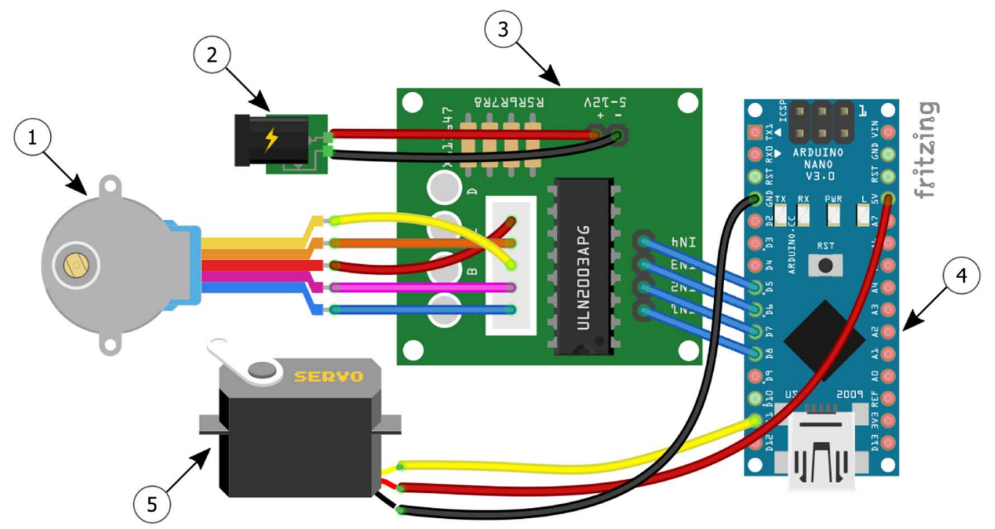

Electronics schematic diagram. The numbers in the figure indicate the following: (1) 28BYJ-48 stepper motor; (2) 1 A, 12 V power supply socket; (3) ULN200xx chip stepper-driver board; (4) Arduino Nano microcontroller; (5) parallax standard servo.

\section{Figure 41}

(a)

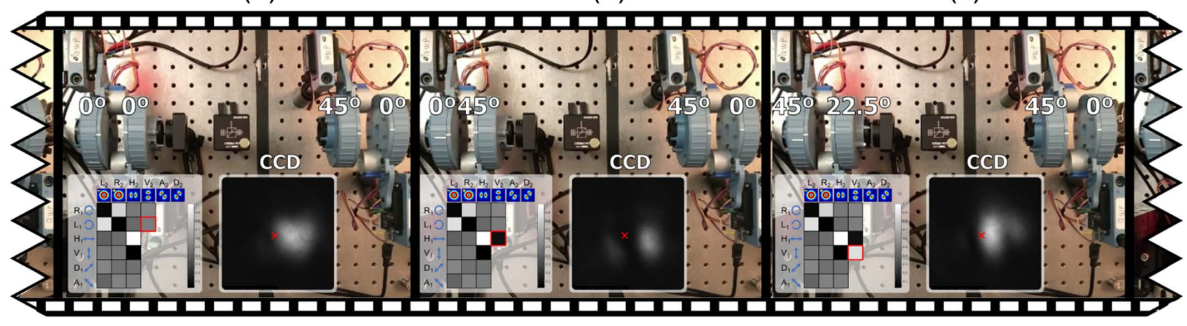

Video demonstration of the automated state-tomography system. (a)-(c) Three angular arrangements of the polarization optics as performed during a tomographic measurement are shown. In the video (see Visualization 1 [126]), we show our automated tomography system in action: both the projected states, as acquired by the CCD camera, and the extracted intensities are displayed, as the system iterates through the 36 programmed angular positions of the polarization optics. 


\section{CONCLUSION AND OUTLOOK}

In this tutorial we have outlined the basic concepts of a quantum state tomography, an essential tool in any quantum laboratory for inferring information on quantum states. We have outlined the ideas using polarization and spatial modes of light as bases, as well as hybrid states of the two. Importantly, we have emphasized that a QST may be simulated with bright classical light in two ways: first, using scalar light in a backprojection approach, in which one detector in the quantum setup is replaced with an intensity laser beam. This allows the entire QST to be mimicked very accurately. Second, we have shown that a QST may be performed without any procedural adjustments with vector beams - classically entangled bright laser beams. In this approach, all the essential features of a QST may be demonstrated, which we believe will be an invaluable tool in teaching experimental quantum science without the complexity of handling single photons. We have 3D printed the core components and automated them with home-built systems, and provide all the necessary detail (designs and code) for this to be repeated by others. We hope that this will inspire the introduction of experimental tomographic measurements in undergraduate quantum courses, while the core of the tutorial will be useful to researchers.

\section{FUNDING}

Engineering and Physical Sciences Research Council (EPSRC) (EP/L016753/1); Claude Leon Foundation.

\section{ACKNOWLEDGMENT}

E. T. thanks Dr. Graham Gibson for useful discussions about rapid prototyping and for logistical support.

These authors contributed equally to this work.

\section{REFERENCES}

1. A. I. Lvovsky and M. G. Raymer, "Continuous-variable optical quantum-state tomography," Rev. Mod. Phys. 81, 299-332 (2009).

2. M. Paris and J. Řeháček, eds., Quantum State Estimation, Vol. 649 of Lecture Notes in Physics (Springer, 2004).

3. G. M. D'Ariano, M. G. Paris, and M. F. Sacchi, "Quantum tomography," Adv. Imaging Electron Phys. 128, 206-309 (2003).

4. D. F. James, P. G. Kwiat, W. J. Munro, and A. G. White, "On the measurement of qubits," in Asymptotic Theory of Quantum Statistical Inference: Selected Papers (World Scientific, 2005), pp. 509-538.

5. M. Schlosshauer, "Decoherence, the measurement problem, and interpretations of quantum mechanics," Rev. Mod. Phys. 76, 1267-1305 (2005).

6. S. Luo, "Using measurement-induced disturbance to characterize correlations as classical or quantum," Phys. Rev. A 77, 022301 (2008).

7. G. M. D'Ariano and H. Yuen, "Impossibility of measuring the wave function of a single quantum system," Phys. Rev. Lett. 76, 2832-2835 (1996).

8. W. K. Wootters and W. H. Zurek, "A single quantum cannot be cloned," Nature 299, 802-803 (1982).

9. A. G. White, D. F. James, P. H. Eberhard, and P. G. Kwiat, "Nonmaximally entangled states: production, characterization, and utilization," Phys. Rev. Lett. 83, 3103-3107 (1999).

10. N. Brunner, S. Pironio, A. Acin, N. Gisin, A. A. Méthot, and V. Scarani, "Testing the dimension of Hilbert spaces," Phys. Rev. Lett. 100, 210503 (2008). 
11. M. Hendrych, R. Gallego, M. Mičuda, N. Brunner, A. Acín, and J. P. Torres, "Experimental estimation of the dimension of classical and quantum systems," Nat. Phys. 8, 588-591 (2012).

12. J. Ahrens, P. Badziag, A. Cabello, and M. Bourennane, "Experimental deviceindependent tests of classical and quantum dimensions," Nat. Phys. 8, 592-595 (2012).

13. L. M. Johansen, "Quantum theory of successive projective measurements," Phys. Rev. A 76, 012119 (2007).

14. J. B. Altepeter, E. R. Jeffrey, and P. G. Kwiat, "Photonic state tomography," Adv. At. Mol. Opt. Phys. 52, 105-159 (2005).

15. L. P. Hughston, R. Jozsa, and W. K. Wootters, "A complete classification of quantum ensembles having a given density matrix," Phys. Lett. A 183, 14-18 (1993).

16. U. Fano, "Description of states in quantum mechanics by density matrix and operator techniques," Rev. Mod. Phys. 29, 74-93 (1957).

17. K. Banaszek, G. D'ariano, M. Paris, and M. Sacchi, "Maximum-likelihood estimation of the density matrix," Phys. Rev. A 61, 010304 (1999).

18. S. R. White, "Density matrix formulation for quantum renormalization groups," Phys. Rev. Lett. 69, 2863-2866 (1992).

19. M. Agnew, J. Leach, M. McLaren, F. S. Roux, and R. W. Boyd, "Tomography of the quantum state of photons entangled in high dimensions," Phys. Rev. A 84, 062101 (2011).

20. K. Banaszek, M. Cramer, and D. Gross, "Focus on quantum tomography," New J. Phys. 15, 125020 (2013).

21. C. H. Bennett, G. Brassard, S. Popescu, B. Schumacher, J. A. Smolin, and W. K. Wootters, "Purification of noisy entanglement and faithful teleportation via noisy channels," Phys. Rev. Lett. 76, 722-725 (1996).

22. E. Knill, R. Laflamme, and L. Viola, "Theory of quantum error correction for general noise," Phys. Rev. Lett. 84, 2525-2528 (2000).

23. P. Samuelsson and M. Büttiker, "Quantum state tomography with quantum shot noise," Phys. Rev. B 73, 041305 (2006).

24. T. Monz, P. Schindler, J. T. Barreiro, M. Chwalla, D. Nigg, W. A. Coish, M. Harlander, W. Hänsel, M. Hennrich, and R. Blatt, "14-qubit entanglement: creation and coherence," Phys. Rev. Lett. 106, 130506 (2011).

25. P. G. Kwiat, K. Mattle, H. Weinfurter, A. Zeilinger, A. V. Sergienko, and Y. Shih, "New high-intensity source of polarization-entangled photon pairs," Phys. Rev. Lett. 75, 4337-4341 (1995).

26. J. Lawrence, Č. Brukner, and A. Zeilinger, "Mutually unbiased binary observable sets on $n$ qubits," Phys. Rev. A 65, 032320 (2002).

27. M. Żukowski and Č. Brukner, "Bell's theorem for general $n$-qubit states," Phys. Rev. Lett. 88, 210401 (2002).

28. M. D. de Burgh, N. K. Langford, A. C. Doherty, and A. Gilchrist, "Choice of measurement sets in qubit tomography," Phys. Rev. A 78, 052122 (2008).

29. H. Zhu, "Quantum state estimation with informationally overcomplete measurements," Phys. Rev. A 90, 012115 (2014).

30. R. T. Thew, K. Nemoto, A. G. White, and W. J. Munro, "Qudit quantum-state tomography," Phys. Rev. A 66, 012303 (2002).

31. D. J. Lum, S. H. Knarr, and J. C. Howell, "Fast Hadamard transforms for compressive sensing of joint systems: measurement of a 3.2 million-dimensional bi-photon probability distribution," Opt. Express 23, 27636-27649 (2015).

32. S. S. Straupe, "Adaptive quantum tomography," JETP Lett. 104, 510-522 (2016). 
33. H. Sosa-Martinez, N. Lysne, C. Baldwin, A. Kalev, I. Deutsch, and P. Jessen, "Experimental study of optimal measurements for quantum state tomography," Phys. Rev. Lett. 119, 150401 (2017).

34. J. Bavaresco, N. H. Valencia, C. Klöckl, M. Pivoluska, P. Erker, N. Friis, M. Malik, and M. Huber, "Measurements in two bases are sufficient for certifying high-dimensional entanglement," Nat. Phys. 14, 1032-1037 (2018).

35. J. G. Titchener, M. Gräfe, R. Heilmann, A. S. Solntsev, A. Szameit, and A. A. Sukhorukov, "Scalable on-chip quantum state tomography," npj Quantum Inf. 4, 19 (2018).

36. K. Wang, J. G. Titchener, S. S. Kruk, L. Xu, H.-P. Chung, M. Parry, I. I. Kravchenko, Y.-H. Chen, A. S. Solntsev, Y. S. Kivshar, D. N. Neshev, and A. A. Sukhorukov, "Quantum metasurface for multi-photon interference and state reconstruction," Science 361, 1104-1108 (2018).

37. L. Banchi, W. S. Kolthammer, and M. Kim, "Multiphoton tomography with linear optics and photon counting," arXiv:1806.02436 (2018).

38. K. Vogel and H. Risken, "Determination of quasiprobability distributions in terms of probability distributions for the rotated quadrature phase," Phys. Rev. A 40, 2847-2849 (1989).

39. E. Wigner, "On the quantum correction for thermodynamic equilibrium," Phys. Rev. 40, 749-759 (1932).

40. M. Hillery, R. F. O'Connell, M. O. Scully, and E. P. Wigner, "Distribution functions in physics: fundamentals," Phys. Rep. 106, 121-167 (1984).

41. W. Band and J. L. Park, "The empirical determination of quantum states," Found. Phys. 1, 133-144 (1970).

42. A. Royer, "Measurement of quantum states and the Wigner function," Found. Phys. 19, 3-32 (1989).

43. G. Birkhoff and J. Von Neumann, "The logic of quantum mechanics," Ann. Math. 37, 823-843 (1936).

44. F. Mallet, M. Castellanos-Beltran, H. Ku, S. Glancy, E. Knill, K. Irwin, G. Hilton, L. Vale, and K. Lehnert, "Quantum state tomography of an itinerant squeezed microwave field," Phys. Rev. Lett. 106, 220502 (2011).

45. M. Crispino, G. Di Giuseppe, F. De Martini, P. Mataloni, and H. Kanatsoulis, "Towards a Fock-states tomographic reconstruction," Fortschr. Phys. 48, 589598 (2000).

46. A. Lvovsky and M. Raymer, "Continuous-variable quantum-state tomography of optical fields and photons," in Quantum Information with Continuous Variables of Atoms and Light (World Scientific, 2007), pp. 409-433.

47. D. Gross, Y.-K. Liu, S. T. Flammia, S. Becker, and J. Eisert, "Quantum state tomography via compressed sensing," Phys. Rev. Lett. 105, 150401 (2010).

48. M. Ghalaii, M. Afsary, S. Alipour, and A. Rezakhani, "Quantum imaging as an ancilla-assisted process tomography," Phys. Rev. A 94, 042102 (2016).

49. K. J. Resch, P. Walther, and A. Zeilinger, "Full characterization of a three-photon Greenberger-Horne-Zeilinger state using quantum state tomography," Phys. Rev. Lett. 94, 070402 (2005).

50. V. Man'ko and O. Man'ko, "Spin state tomography," J. Exp. Theor. Phys. 85, 430-434 (1997).

51. K. Sanaka, K. Kawahara, and T. Kuga, "New high-efficiency source of photon pairs for engineering quantum entanglement," Phys. Rev. Lett. 86, 5620-5623 (2001).

52. W. J. Munro, D. F. James, A. G. White, and P. G. Kwiat, "Maximizing the entanglement of two mixed qubits," Phys. Rev. A 64, 030302 (2001).

53. D. F. V. James, P. G. Kwiat, W. J. Munro, and A. G. White, "Measurement of qubits," Phys. Rev. A 64, 052312 (2001). 
54. Y. Nambu, K. Usami, Y. Tsuda, K. Matsumoto, and K. Nakamura, "Generation of polarization-entangled photon pairs in a cascade of two type-I crystals pumped by femtosecond pulses," Phys. Rev. A 66, 033816 (2002).

55. I. Marcikic, H. De Riedmatten, W. Tittel, H. Zbinden, and N. Gisin, "Longdistance teleportation of qubits at telecommunication wavelengths," Nature 421, 509-513 (2003).

56. J. L. O'Brien, G. J. Pryde, A. G. White, T. C. Ralph, and D. Branning, "Demonstration of an all-optical quantum controlled-NOT gate," Nature 426, 264-267 (2003).

57. T. Yamamoto, M. Koashi, Ş. K. Özdemir, and N. Imoto, "Experimental extraction of an entangled photon pair from two identically decohered pairs," Nature 421, 343-346 (2003).

58. H. Häffner, W. Hänsel, C. Roos, J. Benhelm, M. Chwalla, T. Körber, U. Rapol, M. Riebe, P. Schmidt, C. Becher, O. Gühne, W. Dür, and R. Blatt, "Scalable multiparticle entanglement of trapped ions," Nature 438, 643-646 (2005).

59. D. Leibfried, E. Knill, S. Seidelin, J. Britton, R. B. Blakestad, J. Chiaverini, D. B. Hume, W. M. Itano, J. D. Jost, C. Langer, R. Ozeri, R. Reichle, and D. J. Wineland, "Creation of a six-atom 'Schrödinger cat' state," Nature 438, 639-642 (2005).

60. T. Dunn, I. Walmsley, and S. Mukamel, "Experimental determination of the quantum-mechanical state of a molecular vibrational mode using fluorescence tomography," Phys. Rev. Lett. 74, 884-887 (1995).

61. A. Aspect, P. Grangier, and G. Roger, "Experimental tests of realistic local theories via Bell's theorem," Phys. Rev. Lett. 47, 460-463 (1981).

62. A. Aspect, J. Dalibard, and G. Roger, "Experimental test of Bell's inequalities using time-varying analyzers," Phys. Rev. Lett. 49, 1804-1807 (1982).

63. D. Bouwmeester, J.-W. Pan, K. Mattle, M. Eibl, H. Weinfurter, and A. Zeilinger, "Experimental quantum teleportation," Nature 390, 575-579 (1997).

64. P. G. Kwiat, E. Waks, A. G. White, I. Appelbaum, and P. H. Eberhard, "Ultrabright source of polarization-entangled photons," Phys. Rev. A 60, R773-R776 (1999).

65. A. Chefles, "12 quantum states: discrimination and classical information transmission. A review of experimental progress," in Quantum State Estimation (Springer, 2004), pp. 467-511.

66. Ö. Bayraktar, M. Swillo, C. Canalias, and G. Björk, "Quantum-polarization state tomography," Phys. Rev. A 94, 020105 (2016).

67. A. Mair, A. Vaziri, G. Weihs, and A. Zeilinger, "Entanglement of the orbital angular momentum states of photons," Nature 412, 313-316 (2001).

68. M. McLaren, M. Agnew, J. Leach, F. S. Roux, M. J. Padgett, R. W. Boyd, and A. Forbes, "Entangled Bessel-Gaussian beams," Opt. Express 20, 23589-23597 (2012).

69. M. Krenn, R. Fickler, M. Huber, R. Lapkiewicz, W. Plick, S. Ramelow, and A. Zeilinger, "Entangled singularity patterns of photons in Ince-Gauss modes," Phys. Rev. A 87, 012326 (2013).

70. E. Karimi, R. Boyd, P. De La Hoz, H. De Guise, J. Řeháček, Z. Hradil, A. Aiello, G. Leuchs, and L. L. Sánchez-Soto, "Radial quantum number of Laguerre-Gauss modes," Phys. Rev. A 89, 063813 (2014).

71. V. Salakhutdinov, E. Eliel, and W. Löffler, "Full-field quantum correlations of spatially entangled photons," Phys. Rev. Lett. 108, 173604 (2012).

72. Y. Zhang, S. Prabhakar, C. Rosales-Guzmán, F. S. Roux, E. Karimi, and A. Forbes, "Hong-Ou-Mandel interference of entangled Hermite-Gauss modes," Phys. Rev. A 94, 033855 (2016). 
73. S. Gröblacher, T. Jennewein, A. Vaziri, G. Weihs, and A. Zeilinger, "Experimental quantum cryptography with qutrits," New J. Phys. 8, 75 (2006).

74. M. Mafu, A. Dudley, S. Goyal, D. Giovannini, M. McLaren, M. J. Padgett, T. Konrad, F. Petruccione, N. Lütkenhaus, and A. Forbes, "Higher-dimensional orbital-angular-momentum-based quantum key distribution with mutually unbiased bases," Phys. Rev. A 88, 032305 (2013).

75. M. Mirhosseini, O. S. Magaña-Loaiza, M. N. O’Sullivan, B. Rodenburg, M. Malik, M. P. J. Lavery, M. J. Padgett, D. J. Gauthier, and R. W. Boyd, "High-dimensional quantum cryptography with twisted light," New J. Phys. 17, 033033 (2015).

76. S. Walborn, D. Lemelle, M. Almeida, and P. S. Ribeiro, "Quantum key distribution with higher-order alphabets using spatially encoded qudits," Phys. Rev. Lett. 96, 090501 (2006).

77. D. Collins, N. Gisin, N. Linden, S. Massar, and S. Popescu, "Bell inequalities for arbitrarily high-dimensional systems," Phys. Rev. Lett. 88, 040404 (2002).

78. A. C. Dada, J. Leach, G. S. Buller, M. J. Padgett, and E. Andersson, "Experimental high-dimensional two-photon entanglement and violations of generalized Bell inequalities," Nat. Phys. 7, 677-680 (2011).

79. Y. Zhang, F. S. Roux, T. Konrad, M. Agnew, J. Leach, and A. Forbes, "Engineering two-photon high-dimensional states through quantum interference," Sci. Adv. 2, e1501165 (2016).

80. Y. Zhang, M. Agnew, T. Roger, F. S. Roux, T. Konrad, D. Faccio, J. Leach, and A. Forbes, "Simultaneous entanglement swapping of multiple orbital angular momentum states of light," Nat. Commun. 8, 632 (2017).

81. D. Giovannini, J. Romero, J. Leach, A. Dudley, A. Forbes, and M. J. Padgett, "Characterization of high-dimensional entangled systems via mutually unbiased measurements," Phys. Rev. Lett. 110, 143601 (2013).

82. M. Cramer, M. B. Plenio, S. T. Flammia, R. Somma, D. Gross, S. D. Bartlett, O. Landon-Cardinal, D. Poulin, and Y.-K. Liu, "Efficient quantum state tomography," Nat. Commun. 1, 149 (2010).

83. N. Bent, H. Qassim, A. Tahir, D. Sych, G. Leuchs, L. Sánchez-Soto, E. Karimi, and R. Boyd, "Experimental realization of quantum tomography of photonic qudits via symmetric informationally complete positive operator-valued measures," Phys. Rev. X 5, 041006 (2015).

84. M. Żukowski and A. Zeilinger, "Test of the Bell inequality based on phase and linear momentum as well as spin," Phys. Lett. A 155, 69-72 (1991).

85. X.-S. Ma, A. Qarry, J. Kofler, T. Jennewein, and A. Zeilinger, "Experimental violation of a Bell inequality with two different degrees of freedom of entangled particle pairs," Phys. Rev. A 79, 042101 (2009).

86. L. Neves, G. Lima, A. Delgado, and C. Saavedra, "Hybrid photonic entanglement: realization, characterization, and applications," Phys. Rev. A 80, 042322 (2009).

87. E. Karimi, J. Leach, S. Slussarenko, B. Piccirillo, L. Marrucci, L. Chen, W. She, S. Franke-Arnold, M. J. Padgett, and E. Santamato, "Spin-orbit hybrid entanglement of photons and quantum contextuality," Phys. Rev. A 82, 022115 (2010).

88. D. Richardson, J. Fini, and L. Nelson, "Space-division multiplexing in optical fibres," Nat. Photonics 7, 354-362 (2013).

89. G. Milione, M. P. J. Lavery, H. Huang, Y. Ren, G. Xie, T. A. Nguyen, E. Karimi, L. Marrucci, D. A. Nolan, R. R. Alfano, and A. E. Willner, " $4 \times 20$ Gbit/s mode division multiplexing over free space using vector modes and a q-plate mode (de) multiplexer," Opt. Lett. 40, 1980-1983 (2015).

90. C. Rosales-Guzmán, N. Bhebhe, and A. Forbes, "Simultaneous generation of multiple vector beams on a single SLM," Opt. Express 25, 25697-25706 (2017). 
91. L. Marrucci, C. Manzo, and D. Paparo, "Optical spin-to-orbital angular momentum conversion in inhomogeneous anisotropic media," Phys. Rev. Lett. 96, 163905 (2006).

92. R. C. Devlin, A. Ambrosio, D. Wintz, S. L. Oscurato, A. Y. Zhu, M. Khorasaninejad, J. Oh, P. Maddalena, and F. Capasso, "Spin-to-orbital angular momentum conversion in dielectric metasurfaces," Opt. Express 25, 377-393 (2017).

93. R. C. Devlin, A. Ambrosio, N. A. Rubin, J. B. Mueller, and F. Capasso, "Arbitrary spin-to-orbital angular momentum conversion of light," Science 358, 896-901 (2017).

94. E. Nagali, F. Sciarrino, F. De Martini, L. Marrucci, B. Piccirillo, E. Karimi, and E. Santamato, "Quantum information transfer from spin to orbital angular momentum of photons," Phys. Rev. Lett. 103, 013601 (2009).

95. B. Gadway, E. Galvez, and F. De Zela, "Bell-inequality violations with single photons entangled in momentum and polarization," J. Phys. B 42, 015503 (2009).

96. K. H. Kagalwala, G. Di Giuseppe, A. F. Abouraddy, and B. E. Saleh, "Bell's measure in classical optical coherence," Nat. Photonics 7, 72-78 (2013).

97. B. Ndagano, R. Brüning, M. McLaren, M. Duparré, and A. Forbes, "Fiber propagation of vector modes," Opt. Express 23, 17330-17336 (2015).

98. E. Otte, I. Nape, C. Rosales-Guzmán, A. Vallés, C. Denz, and A. Forbes, "Recovery of local entanglement in self-healing vector vortex Bessel beams," arXiv:1805.08179 (2018).

99. M. Raymer, M. Beck, and D. McAlister, "Complex wave-field reconstruction using phase-space tomography," Phys. Rev. Lett. 72, 1137-1140 (1994).

100. D. McAlister, M. Beck, L. Clarke, A. Mayer, and M. Raymer, "Optical phase retrieval by phase-space tomography and fractional-order Fourier transforms," Opt. Lett. 20, 1181-1183 (1995).

101. A. Luis, "Coherence, polarization, and entanglement for classical light fields," Opt. Commun. 282, 3665-3670 (2009).

102. C. Borges, M. Hor-Meyll, J. Huguenin, and A. Khoury, "Bell-like inequality for the spin-orbit separability of a laser beam,” Phys. Rev. A 82, 033833 (2010).

103. G. Milione, T. A. Nguyen, J. Leach, D. A. Nolan, and R. R. Alfano, "Using the nonseparability of vector beams to encode information for optical communication," Opt. Lett. 40, 4887-4890 (2015).

104. B. Ndagano, B. Perez-Garcia, F. S. Roux, M. McLaren, C. Rosales-Guzman, Y. Zhang, O. Mouane, R. I. Hernandez-Aranda, T. Konrad, and A. Forbes, "Characterizing quantum channels with non-separable states of classical light," Nat. Phys. 13, 397-402 (2017).

105. X.-F. Qian, B. Little, J. C. Howell, and J. Eberly, "Shifting the quantum-classical boundary: theory and experiment for statistically classical optical fields," Optica 2, 611-615 (2015).

106. X.-F. Qian, A. N. Vamivakas, and J. H. Eberly, "Emerging connections: classical and quantum optics," Opt. Photon. News 28(10), 34-41 (2017).

107. X.-F. Qian and J. Eberly, "Entanglement and classical polarization states," Opt. Lett. 36, 4110-4112 (2011).

108. P. Ghose and A. Mukherjee, "Entanglement in classical optics," Rev. Theor. Sci. 2, 274-288 (2014).

109. F. Töppel, A. Aiello, C. Marquardt, E. Giacobino, and G. Leuchs, "Classical entanglement in polarization metrology," New J. Phys. 16, 073019 (2014).

110. B. N. Simon, S. Simon, F. Gori, M. Santarsiero, R. Borghi, N. Mukunda, and R. Simon, "Nonquantum entanglement resolves a basic issue in polarization optics," Phys. Rev. Lett. 104, 023901 (2010). 
111. C. Samlan and N. K. Viswanathan, "Generation of vector beams using a doublewedge depolarizer: non-quantum entanglement," Opt. Lasers Eng. 82, 135-140 (2016).

112. K. Subramanian and N. K. Viswanathan, "Measuring correlations in nonseparable vector beams using projective measurements," Opt. Commun. 399, 45-51 (2017).

113. A. Aiello, F. Töppel, C. Marquardt, E. Giacobino, and G. Leuchs, "Quantum-like nonseparable structures in optical beams," New J. Phys. 17, 043024 (2015).

114. R. J. Spreeuw, "Classical wave-optics analogy of quantum-information processing," Phys. Rev. A 63, 062302 (2001).

115. T. Pittman, Y. Shih, D. Strekalov, and A. Sergienko, "Optical imaging by means of two-photon quantum entanglement," Phys. Rev. A 52, R3429 (1995).

116. D. Strekalov, A. Sergienko, D. Klyshko, and Y. Shih, "Observation of twophoton 'ghost' interference and diffraction," Phys. Rev. Lett. 74, 3600-3603 (1995).

117. T. Pittman, D. Strekalov, D. Klyshko, M. Rubin, A. Sergienko, and Y. Shih, “Two-photon geometric optics," Phys. Rev. A 53, 2804-2815 (1996).

118. M. McLaren, J. Romero, M. J. Padgett, F. S. Roux, and A. Forbes, "Two-photon optics of Bessel-Gaussian modes," Phys. Rev. A 88, 033818 (2013).

119. R. S. Aspden, D. S. Tasca, A. Forbes, R. W. Boyd, and M. J. Padgett, "Experimental demonstration of Klyshko's advanced-wave picture using a coincidence-count based, camera-enabled imaging system," J. Mod. Opt. 61, 547-551 (2014).

120. S. Oemrawsingh, J. de Jong, X. Ma, A. Aiello, E. Eliel, and J. Woerdman, "Highdimensional mode analyzers for spatial quantum entanglement," Phys. Rev. A 73, 032339 (2006).

121. Y. Zhang, M. McLaren, F. S. Roux, and A. Forbes, "Simulating quantum state engineering in spontaneous parametric down-conversion using classical light," Opt. Express 22, 17039-17049 (2014).

122. D. Klyshko, "A simple method of preparing pure states of an optical field, of implementing the Einstein-Podolsky-Rosen experiment, and of demonstrating the complementarity principle," Sov. Phys. Usp. 31, 74-85 (1988).

123. G. Milione, H. I. Sztul, D. A. Nolan, and R. R. Alfano, "Higher-order Poincaré sphere, Stokes parameters, and the angular momentum of light," Phys. Rev. Lett. 107, 053601 (2011).

124. A. Holleczek, A. Aiello, C. Gabriel, C. Marquardt, and G. Leuchs, "Classical and quantum properties of cylindrically polarized states of light," Opt. Express 19, 9714-9736 (2011).

125. G. Milione, S. Evans, D. A. Nolan, and R. R. Alfano, "Higher order Pancharatnam-Berry phase and the angular momentum of light," Phys. Rev. Lett. 108, 190401 (2012).

126. Supplemental material to this publication including the video of the system in action, 3D-designs of the roto-flip stages, the Arduino firmware, and the LabVIEW programs, can be found at the following links: https://doi.org/10 .6084/m9.figshare.7035506, https://doi.org/10.6084/m9.figshare.7035509, and https://doi.org/10.6084/m9.figshare.7035518.

127. R. A. Beth, "Mechanical detection and measurement of the angular momentum of light," Phys. Rev. 50, 115-125 (1936).

128. R. Schmied, "Quantum state tomography of a single qubit: comparison of methods," J. Mod. Opt. 63, 1744-1758 (2016).

129. S. Barnett, Quantum Information (Oxford University, 2009), Vol. 16.

130. A. Forbes, A. Dudley, and M. McLaren, "Creation and detection of optical modes with spatial light modulators," Adv. Opt. Photon. 8, 200-227 (2016). 
131. S. Franke-Arnold, L. Allen, and M. Padgett, "Advances in optical angular momentum," Laser Photon. Rev. 2, 299-313 (2008).

132. A. M. Yao and M. J. Padgett, "Orbital angular momentum: origins, behavior and applications," Adv. Opt. Photon. 3, 161-204 (2011).

133. A. E. Willner, H. Huang, Y. Yan, Y. Ren, N. Ahmed, G. Xie, C. Bao, L. Li, Y. Cao, Z. Zhao, J. Wang, M. P. J. Lavery, M. Tur, S. Ramachandran, A. F. Molisch, N. Ashrafi, and S. Ashrafi, "Optical communications using orbital angular momentum beams," Adv. Opt. Photon. 7, 66-106 (2015).

134. H. Rubinsztein-Dunlop, A. Forbes, M. V. Berry, M. R. Dennis, D. L. Andrews, M. Mansuripur, C. Denz, C. Alpmann, P. Banzer, T. Bauer, E. Karimi, L. Marrucci, M. Padgett, M. Ritsch-Marte, N. M. Litchinitser, N. P. Bigelow, C. Rosales-Guzmán, A. Belmonte, J. P. Torres, T. W. Neely, M. Baker, R. Gordon, A. B. Stilgoe, J. Romero, A. G. White, R. Fickler, A. E. Willner, G. Xie, B. McMorran, and A. M. Weiner, "Roadmap on structured light," J. Opt. 19, 013001 (2017).

135. A. E. Willner, Y. Ren, G. Xie, Y. Yan, L. Li, Z. Zhao, J. Wang, M. Tur, A. F. Molisch, and S. Ashrafi, "Recent advances in high-capacity free-space optical and radio-frequency communications using orbital angular momentum multiplexing," Philos. Trans. R. Soc. London Ser. A 375, 20150439 (2017).

136. M. Krenn, M. Malik, M. Erhard, and A. Zeilinger, "Orbital angular momentum of photons and the entanglement of Laguerre-Gaussian modes," Philos. Trans. R. Soc. London Ser. A 375, 20150442 (2017).

137. M. Erhard, R. Fickler, M. Krenn, and A. Zeilinger, "Twisted photons: new quantum perspectives in high dimensions," Light Sci. Appl. 7, 17146 (2018).

138. A. Sit, F. Bouchard, R. Fickler, J. Gagnon-Bischoff, H. Larocque, K. Heshami, D. Elser, C. Peuntinger, K. Gunthner, B. Heim, C. Marquardt, G. Leuchs, R. W. Boyd, and E. Karimi, "High-dimensional intracity quantum cryptography with structured photons," Optica 4, 1006-1010 (2017).

139. F. Bouchard, R. Fickler, R. W. Boyd, and E. Karimi, "High-dimensional quantum cloning and applications to quantum hacking," Sci. Adv. 3, e1601915 (2017).

140. M. J. Padgett and J. Courtial, "Poincaré-sphere equivalent for light beams containing orbital angular momentum," Opt. Lett. 24, 430-432 (1999).

141. L. Allen, M. W. Beijersbergen, R. Spreeuw, and J. Woerdman, "Orbital angular momentum of light and the transformation of Laguerre-Gaussian laser modes," Phys. Rev. A 45, 8185-8189 (1992).

142. B. Jack, J. Leach, H. Ritsch, S. M. Barnett, M. J. Padgett, and S. Franke-Arnold, "Precise quantum tomography of photon pairs with entangled orbital angular momentum," New J. Phys. 11, 103024 (2009).

143. D. Flamm, D. Naidoo, C. Schulze, A. Forbes, and M. Duparré, "Mode analysis with a spatial light modulator as a correlation filter," Opt. Lett. 37, 2478-2480 (2012).

144. D. Flamm, C. Schulze, D. Naidoo, S. Schroter, A. Forbes, and M. Duparre, "Alldigital holographic tool for mode excitation and analysis in optical fibers," J. Lightwave Technol. 31, 1023-1032 (2013).

145. C. Schulze, S. Ngcobo, M. Duparré, and A. Forbes, "Modal decomposition without a priori scale information," Opt. Express 20, 27866-27873 (2012).

146. C. Schulze, A. Dudley, D. Flamm, M. Duparré, and A. Forbes, "Measurement of the orbital angular momentum density of light by modal decomposition," New J. Phys. 15, 073025 (2013).

147. A. Dudley, Y. Li, T. Mhlanga, M. Escuti, and A. Forbes, "Generating and measuring nondiffracting vector Bessel beams," Opt. Lett. 38, 3429-3432 (2013).

148. C. Rosales-Guzmán and A. Forbes, How to Shape Light with Spatial Light Modulators (SPIE, 2017). 
149. R. Horodecki, P. Horodecki, M. Horodecki, and K. Horodecki, "Quantum entanglement," Rev. Mod. Phys. 81, 865-942 (2009).

150. A. Vallés, V. D’Ambrosio, M. Hendrych, M. Mičuda, L. Marrucci, F. Sciarrino, and J. P. Torres, "Generation of tunable entanglement and violation of a Bell-like inequality between different degrees of freedom of a single photon," Phys. Rev. A 90, 052326 (2014).

151. I. Nape, B. Ndagano, and A. Forbes, "Erasing the orbital angular momentum information of a photon,” Phys. Rev. A 95, 053859 (2017).

152. M. McLaren, T. Konrad, and A. Forbes, "Measuring the nonseparability of vector vortex beams," Phys. Rev. A 92, 023833 (2015).

153. B. Ndagano, H. Sroor, M. McLaren, C. Rosales-Guzmán, and A. Forbes, "Beam quality measure for vector beams," Opt. Lett. 41, 3407-3410 (2016).

154. R. Jozsa, "Fidelity for mixed quantum states," J. Mod. Opt. 41, 2315-2323 (1994).

155. S. Hill and W. K. Wootters, "Entanglement of a pair of quantum bits," Phys. Rev. Lett. 78, 5022-5025 (1997).

156. M. McLaren, T. Mhlanga, M. J. Padgett, F. S. Roux, and A. Forbes, "Self-healing of quantum entanglement after an obstruction," Nat. Commun. 5, 3248 (2014).

157. M. Arruda, W. Soares, S. Walborn, D. Tasca, A. Kanaan, R. M. de Araújo, and P. Ribeiro, "Klyshko's advanced-wave picture in stimulated parametric downconversion with a spatially structured pump beam," Phys. Rev. A 98, 023850 (2018).

158. A. I. Lvovsky and T. Aichele, "Conditionally prepared photon and quantum imaging," Proc. SPIE 5551, 1-7 (2004).

159. R. Meyers, K. S. Deacon, and Y. Shih, "Ghost-imaging experiment by measuring reflected photons," Phys. Rev. A 77, 041801 (2008).

160. M. McLaren and A. Forbes, "Digital spiral-slit for bi-photon imaging," J. Opt. 19, 044006 (2017).

161. A. F. Abouraddy, P. R. Stone, A. V. Sergienko, B. E. Saleh, and M. C. Teich, "Entangled-photon imaging of a pure phase object," Phys. Rev. Lett. 93, 213903 (2004).

162. P.-A. Moreau, E. Toninelli, T. Gregory, and M. J. Padgett, "Ghost imaging using optical correlations," Laser Photon. Rev. 12, 1700143 (2018).

163. J. F. Clauser, M. A. Horne, A. Shimony, and R. A. Holt, "Proposed experiment to test local hidden-variable theories," Phys. Rev. Lett. 23, 880-884 (1969).

164. E. Karimi and R. W. Boyd, "Classical entanglement?" Science 350, 1172-1173 (2015).

165. L. J. Pereira, A. Z. Khoury, and K. Dechoum, "Quantum and classical separability of spin-orbit laser modes," Phys. Rev. A 90, 053842 (2014).

166. Q. Zhan, "Cylindrical vector beams: from mathematical concepts to applications," Adv. Opt. Photon. 1, 1-57 (2009).

167. B. Ndagano, I. Nape, M. A. Cox, C. Rosales-Guzman, and A. Forbes, "Creation and detection of vector vortex modes for classical and quantum communication," J. Lightwave Technol. 36, 292-301 (2018).

168. C. Rosales-Guzmán, B. Ndagano, and A. Forbes, "A review of complex vector light fields and their applications," J. Opt. 20, 123001 (2018).

169. C. Maurer, A. Jesacher, S. Fürhapter, S. Bernet, and M. Ritsch-Marte, "Tailoring of arbitrary optical vector beams," New J. Phys. 9, 78 (2007).

170. D. Naidoo, F. S. Roux, A. Dudley, I. Litvin, B. Piccirillo, L. Marrucci, and A. Forbes, "Controlled generation of higher-order Poincaré sphere beams from a laser," Nat. Photonics 10, 327-332 (2016). 
171. Z. Bomzon, G. Biener, V. Kleiner, and E. Hasman, "Radially and azimuthally polarized beams generated by space-variant dielectric subwavelength gratings," Opt. Lett. 27, 285-287 (2002).

172. K. Y. Bliokh, F. J. Rodrguez-Fortuño, F. Nori, and A. V. Zayats, "Spin-orbit interactions of light," Nat. Photonics 9, 796-808 (2015).

173. F. Cardano and L. Marrucci, "Spin-orbit photonics," Nat. Photonics 9, 776-778 (2015).

174. F. Cardano, E. Karimi, S. Slussarenko, L. Marrucci, C. de Lisio, and E. Santamato, "Polarization pattern of vector vortex beams generated by q-plates with different topological charges," Appl. Opt. 51, C1-C6 (2012).

175. M. G. Nassiri and E. Brasselet, "Multispectral management of the photon orbital angular momentum," Phys. Rev. Lett. 121, 213901 (2018).

176. E. Brasselet, "Tunable high-resolution macroscopic self-engineered geometric phase optical elements," Phys. Rev. Lett. 121, 033901 (2018).

177. M. Rafayelyan and E. Brasselet, "Spin-to-orbital angular momentum mapping of polychromatic light," Phys. Rev. Lett. 120, 213903 (2018).

178. E. Brasselet, N. Murazawa, H. Misawa, and S. Juodkazis, "Optical vortices from liquid crystal droplets," Phys. Rev. Lett. 103, 103903 (2009).

179. N. Shitrit, I. Yulevich, E. Maguid, D. Ozeri, D. Veksler, V. Kleiner, and E. Hasman, "Spin-optical metamaterial route to spin-controlled photonics," Science 340, 724-726 (2013).

180. R. C. Devlin, M. Khorasaninejad, W. T. Chen, J. Oh, and F. Capasso, "Broadband high-efficiency dielectric metasurfaces for the visible spectrum," Proc. Natl. Acad. Sci. USA 113, 10473-10478 (2016).

181. A. Ambrosio, "Structuring visible light with dielectric metasurfaces," J. Opt. 20, 113002 (2018).

182. E. Hasman, G. Biener, A. Niv, and V. Kleiner, "Space-variant polarization manipulation," Prog. Opt. 47, 215-289 (2005).

183. N. F. Yu and F. Capasso, "Flat optics with designer metasurfaces," Nat. Mater. 13, 139-150 (2014).

184. F. Aieta, P. Genevet, M. A. Kats, N. Yu, R. Blanchard, Z. Gaburro, and F. Capasso, "Aberration-free ultrathin flat lenses and axicons at telecom wavelengths based on plasmonic metasurfaces," Nano Lett. 12, 4932-4936 (2012).

185. D. Lin, P. Fan, E. Hasman, and M. L. Brongersma, "Dielectric gradient metasurface optical elements," Science 345, 298-302 (2014).

186. N. F. Yu, P. Genevet, M. A. Kats, F. Aieta, J. P. Tetienne, F. Capasso, and Z. Gaburro, "Light propagation with phase discontinuities: generalized laws of reflection and refraction," Science 334, 333-337 (2011).

187. A. Arbabi, Y. Horie, M. Bagheri, and A. Faraon, "Dielectric metasurfaces for complete control of phase and polarization with subwavelength spatial resolution and high transmission," Nat. Nanotechnol. 10, 937-943 (2015).

188. M. J. Escuti, J. Kim, and M. W. Kudenov, "Controlling light with geometricphase holograms," Opt. Photon. News 27(2), 22-29 (2016).

189. Y. Bromberg, Y. Lahini, R. Morandotti, and Y. Silberberg, "Quantum and classical correlations in waveguide lattices," Phys. Rev. Lett. 102, 253904 (2009).

190. R. Keil, A. Szameit, F. Dreisow, M. Heinrich, S. Nolte, and A. Tünnermann, "Photon correlations in two-dimensional waveguide arrays and their classical estimate," Phys. Rev. A 81, 023834 (2010).

191. R. Keil, F. Dreisow, M. Heinrich, A. Tünnermann, S. Nolte, and A. Szameit, "Classical characterization of biphoton correlation in waveguide lattices," Phys. Rev. A 83, 013808 (2011). 
192. H. Sroor, N. Lisa, D. Naidoo, I. Litvin, and A. Forbes, "Purity of vector vortex beams through a birefringent amplifier," Phys. Rev. Appl. 9, 044010 (2018).

193. P. Li, S. Zhang, and X. Zhang, "Classically high-dimensional correlation: simulation of high-dimensional entanglement," Opt. Express 26, 31413-31429 (2018).

194. X. Yi, Y. Liu, X. Ling, X. Zhou, Y. Ke, H. Luo, S. Wen, and D. Fan, "Hybridorder Poincaré sphere," Phys. Rev. A 91, 023801 (2015).

195. B. Ndagano, I. Nape, B. Perez-Garcia, S. Scholes, R. I. Hernandez-Aranda, T. Konrad, M. P. J. Lavery, and A. Forbes, "A deterministic detector for vector vortex states," Sci. Rep. 7, 13882 (2017).

196. G. Milione, A. Dudley, T. A. Nguyen, O. Chakraborty, E. Karimi, A. Forbes, and R. R. Alfano, "Measuring the self-healing of the spatially inhomogeneous states of polarization of vector Bessel beams," J. Opt. 17, 035617 (2015).

197. G. Vallone, V. D'Ambrosio, A. Sponselli, S. Slussarenko, L. Marrucci, F. Sciarrino, and P. Villoresi, "Free-space quantum key distribution by rotationinvariant twisted photons," Phys. Rev. Lett. 113, 060503 (2014).

198. R. Fickler, R. Lapkiewicz, S. Ramelow, and A. Zeilinger, "Quantum entanglement of complex photon polarization patterns in vector beams," Phys. Rev. A 89, 060301 (2014).

199. S. Berg-Johansen, F. Töppel, B. Stiller, P. Banzer, M. Ornigotti, E. Giacobino, G. Leuchs, A. Aiello, and C. Marquardt, "Classically entangled optical beams for high-speed kinematic sensing," Optica 2, 864-868 (2015).

200. P. Li, B. Wang, and X. Zhang, "High-dimensional encoding based on classical nonseparability," Opt. Express 24, 15143-15159 (2016).

201. L. Marrucci, E. Karimi, S. Slussarenko, B. Piccirillo, E. Santamato, E. Nagali, and F. Sciarrino, "Spin-to-orbital conversion of the angular momentum of light and its classical and quantum applications," J. Opt. 13, 064001 (2011).

202. E. Nagali, F. Sciarrino, F. De Martini, B. Piccirillo, E. Karimi, L. Marrucci, and E. Santamato, "Polarization control of single photon quantum orbital angular momentum states," Opt. Express 17, 18745-18759 (2009).

203. E. Nagali, L. Sansoni, L. Marrucci, E. Santamato, and F. Sciarrino, "Experimental generation and characterization of single-photon hybrid ququarts based on polarization and orbital angular momentum encoding," Phys. Rev. A 81, 052317 (2010).

204. V. D’Ambrosio, E. Nagali, S. P. Walborn, L. Aolita, S. Slussarenko, L. Marrucci, and F. Sciarrino, "Complete experimental toolbox for alignment-free quantum communication," Nat. Commun. 3, 961 (2012).

205. G. Vallone, V. D’Ambrosio, A. Sponselli, S. Slussarenko, L. Marrucci, F. Sciarrino, and P. Villoresi, "Free-space quantum key distribution by rotationinvariant twisted photons," Phys. Rev. Lett. 113, 060503 (2014).

206. A. Forbes, Laser Beam Propagation: Generation and Propagation of Customized Light (CRC Press, 2014).

207. J. S. Bell, "On the problem of hidden variables in quantum mechanics," Rev. Mod. Phys. 38, 447-452 (1966).

208. J. Leach, B. Jack, J. Romero, M. Ritsch-Marte, R. Boyd, A. Jha, S. Barnett, S. Franke-Arnold, and M. Padgett, "Violation of a Bell inequality in two-dimensional orbital angular momentum state-spaces," Opt. Express 17, 8287-8293 (2009).

209. M. Delmans and J. Haseloff, " $\mu$ Cube: a framework for 3D printable optomechanics," J. Open Hardware 2, 2 (2018). 
210. L. J. Salazar-Serrano, J. P. Torres, and A. Valencia, "A 3D printed toolbox for opto-mechanical components," PloS One 12, e0169832 (2017).

211. J. P. Sharkey, D. C. W. Foo, A. Kabla, J. J. Baumberg, and R. W. Bowman, "A one-piece 3D printed flexure translation stage for open-source microscopy," Rev. Sci. Instrum. 87, 025104 (2016).

212. A. P. Zwicker, J. Bloom, R. Albertson, and S. Gershman, "The suitability of 3D printed plastic parts for laboratory use," Am. J. Phys. 83, 281-285 (2015).

213. M. A. Hossain, J. Canning, K. Cook, and A. Jamalipour, "Smartphone laser beam spatial profiler," Opt. Lett. 40, 5156-5159 (2015).

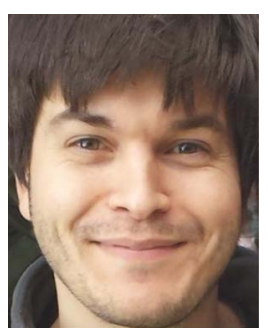

Ermes Toninelli enjoys doing research in the Optics Group at the University of Glasgow, which he joined in 2014 for his Ph.D. studies, and continuing since October 2018 as a postdoctoral researcher. His current research interests are in single-photon imaging and sensing, orbital angular momentum (acoustic and optical), and the development of novel imaging and sensing techniques, both in the classical and quantum regimes.

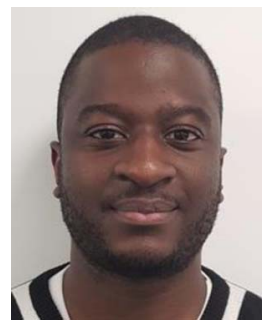

Bienvenu Ndagano completed his Ph.D. at the University of the Witwatersrand, where he worked on applications of classical and quantum entanglement to quantum communication with structured photons. His research has produced 13 peer-reviewed journal articles and 8 conference proceedings. Bienvenu holds a M.Sc. and B.Sc. in Physics from the University of the Witwatersrand and coled the organizing committee for OSA-IONS hosted in South Africa in October 2018. Bienvenu has now moved to the University of Glasgow as a postdoctoral researcher in the Extreme Light group.

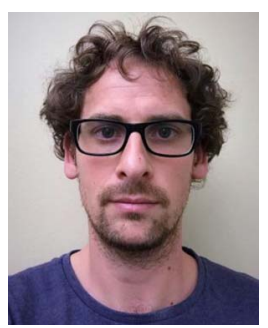

Adam Vallés obtained his B.Sc. in telecom engineering by the Polytechnic University of Catalonia. He then changed course, conducting his master research in photonics, and his Ph.D. based on studying the relation between entanglement, Bell's inequalities and coherence, at the Institute of Photonic Sciences (ICFO). He has been awarded with a postdoctoral fellowship by the Claude Leon Foundation to work in the structured light group from the University of the Witwatersrand. His research at Wits has been focused on high-dimensional quantum communication and quantum imaging.

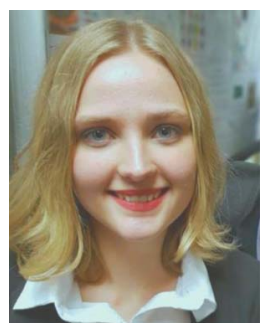

Bereneice Sephton recieved her B.Sc. (Hons.) in physics from Nelson Mandela Metropolitan University in 2016 and M.Sc. from the University of Witwatersrand (Wits) in 2018, where she investigated the realization of Quantum Walks with classical light. She is now pursuing a doctorate in quantum imaging, looking to explore the extremes of current techniques. 


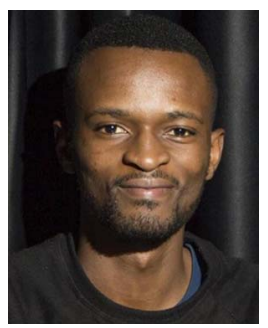

Isaac Mphele Nape graduated from the University of Pretoria and is currently a Ph.D. student at the Wits Structured Light Laboratory in the School of Physics based at the University of the Witwatersrand (South Africa). His research interests include tailoring light's transverse spatial structure for applications in high dimensional quantum information and communication.

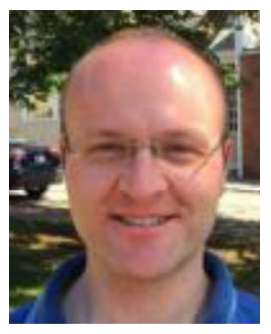

Antonio Ambrosio received his Master's Degree in Condensed Matter Physics from the University of Napoli "Federico II", Italy. In 2006, he received his Ph.D. degree in Applied Physics from the University of Pisa, Italy. From 2006-2013 he worked at Consiglio Nazionale delle Ricerche (CNR), the Italian Research Council, focusing on developing high-resolution optical microscopy techniques and investigating the light-driven surface structuring of azobenzene-containing polymer films. In April 2013, Dr. Ambrosio started collaborating with Prof. Federico Capasso's group at Harvard University as a Visiting Research Scholar of the John A. Paulson School of engineering and Applied Sciences. At Harvard, Dr. Ambrosio built a nano-imaging spectroscopical facility that allows optical imaging with $50 \mathrm{~nm}$ resolution in a broad wavelength range (from $450 \mathrm{~nm}$ to $1.7 \mathrm{um}$ ), for instance, the steering of surface plasmon polaritons in one- and two-dimensional metamaterials. At Harvard, Dr. Ambrosio also started working on dielectric metasurfaces, which allow controlling light in its amplitude, phase, and polarization in ways that are not reproducible with standard optical components. Since July 2016, Dr. Ambrosio has been the Principal Scientist at the Center for Nanoscale Systems at Harvard University, where he has established the Optical Nano-imaging Lab that he is leading in research activity about the development of new optical near-field imaging and spectroscopy techniques for 2D materials, polymers, and nanostructured surfaces.

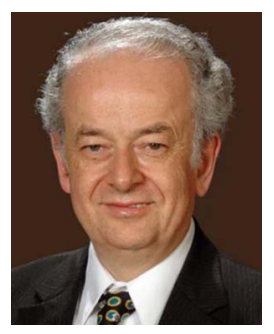

Federico Capasso is the Robert Wallace Professor of Applied Physics at Harvard University, which he joined in 2003 after 27 years at Bell Labs, where he rose from postdoc to VP of Physical Research. He pioneered bandgap engineering of semiconductors, including the invention of the quantum cascade laser, and the field of flat optics with metasurfaces. He carried out high precision measurements of the Casimir force with MEMS and the first measurement of the repulsive Casimir-Lifshitz force. His awards include the Fermi Prize of the Italian Physical Society, the Balzan prize for Applied Photonics, the King Faisal Prize for Science, the IEEE Edison Medal, the APS Arthur Schawlow Prize, the OSA Wood prize, the SPIE Gold Medal, the Rumford Prize of the American Academy of Arts and Sciences, the Franklin Institute Wetherill Medal, and the Materials Research Society Medal. He is a member of the National Academy of Sciences, the National Academy of Engineering, and the American Academy of Arts and Sciences. 


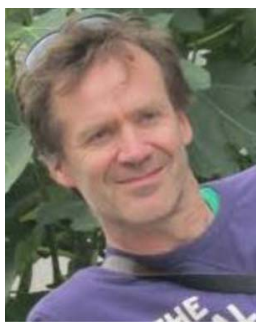

Miles Padgett holds the Kelvin Chair of Natural Philosophy at the University of Glasgow. He is fascinated by light both classical and quantum-specifically light's momentum. In 2001 he was elected to Fellowship of the Royal Society of Edinburgh and in 2014 the Royal Society, the UK's National Academy. In 2009, with Les Allen, he won the IoP Young Medal, in 2014 the RSE Kelvin Medal, in 2015 the Science of Light Prize from the EPS, and in 2017 the Max Born Award of the OSA.

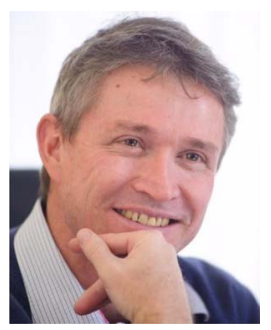

Andrew Forbes received his Ph.D. (1998) from the University of Natal (South Africa) and subsequently spent several years as an applied laser physicist, including in a private laser company at which he was Technical Director and later as Chief Researcher and Research Group Leader of the Mathematical Optics group at the CSIR. Andrew is presently a Distinguished Professor within the School of Physics at the U. Witwatersrand (South Africa), where he has established a new laboratory for Structured Light. Andrew is active in promoting photonics in Africa, a founding member of the Photonics Initiative of South Africa, a Fellow of both SPIE and the OSA, and an elected member of the Academy of Science of South Africa. He spends his time having fun with the taxpayers' money, exploring structured light in lasers, quantum optics, and classical optics. 